$\angle 60139(96 / 50) 2-2 \angle 1-007 \angle-08$

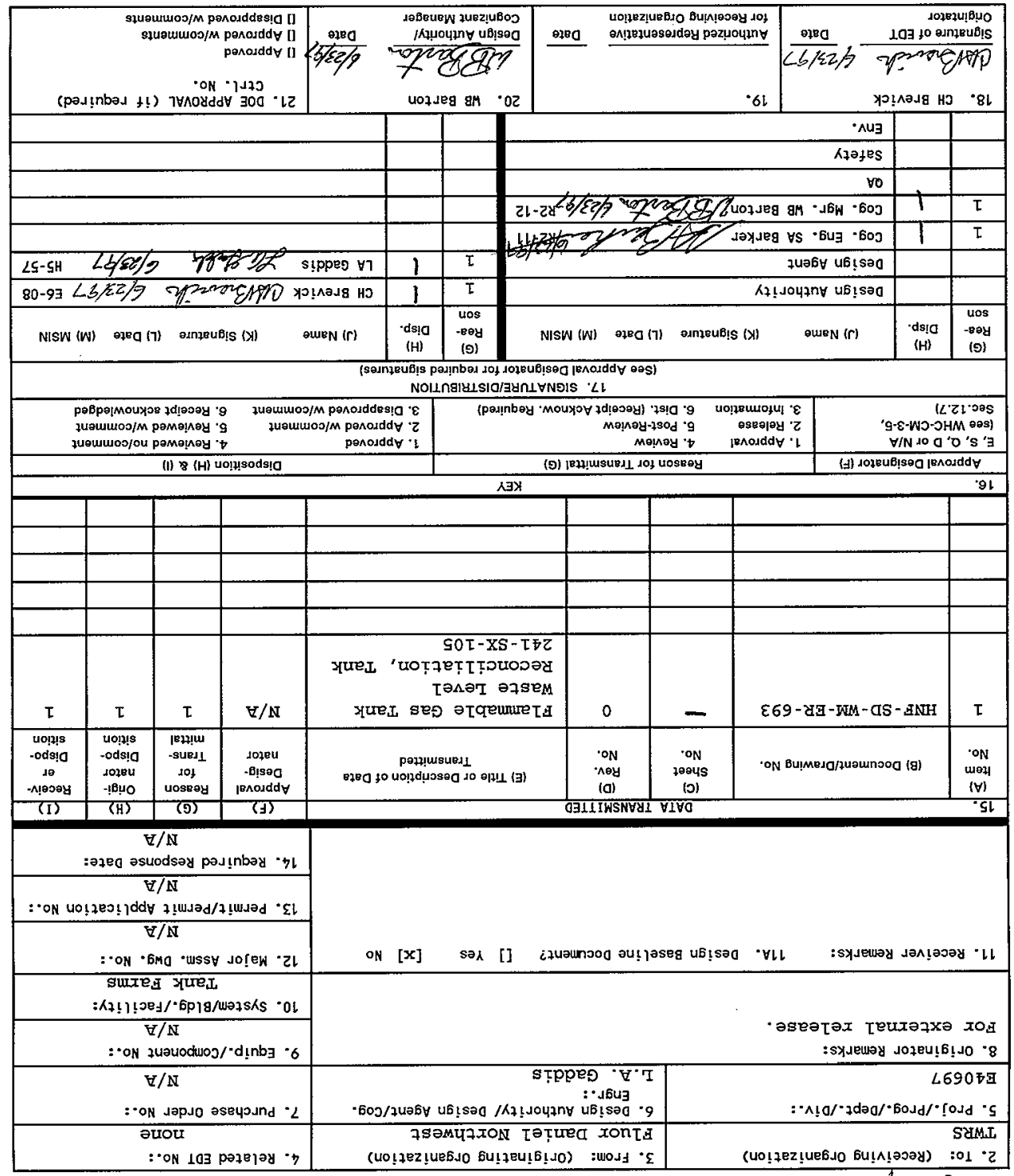




\title{
Flammable Gas Tank Waste Level Reconciliation Tank 241-SX-105
}

\author{
C.H. Brevick, L.A. Gaddis \\ Fluor Daniel Northwest, Richland, WA 99352 \\ U.S. Department of Energy Contract DE-AC06-96RL13200 \\ EDT/ECN: 156939 \\ UC: 2070 \\ Org Code: 408 \\ B\&R Code: EW3120074 \\ Charge Code: B40697 \\ Total pages: $7 \theta^{\circ} 71$ \\ gm 6/24/97
}

Key Words: Flammable gas, waste level, level reconciliation, SX Farm, unexplained increases, slurry growth, hydrogen gas

Abstract: This document provides verification/reconciliation of unexplained waste level increases for tank $241-\mathrm{SX}-105$ as reported in Waste Storage Tank Status and Leak Detection Cxiteria, Vol. 1 and 2, WHC-SD-WM-TI-356 also known as the Welty Report. Waste level increases are studied during the period from 1973 to 1980 . Historical records were used to provide the verification/reconciliation.

TRADEMARK DISCLAIMER. Reference herein to any specific comercial product, process, or service by trade name, trademark, manufacturer, or otherwise, does not necessarily constitute or imply its endorsement, recommendation, or favoring by the United States Government or any agency thereof or its contractors or subcontractors.

Printed in the United States of America. To obtain copies of this document, contact: Document Control Services, P.0. Box 950, Mailstop H6-08, Richland WA 99352, Phone (509) 372-2420; Fax (509) 376-4989.
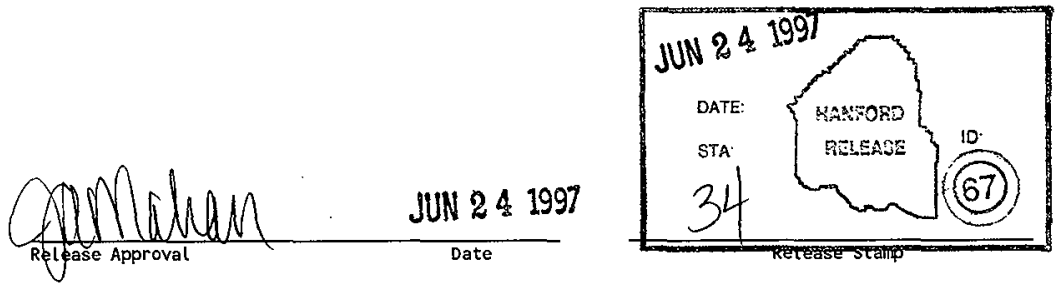


\section{LETTER REPORT}

\section{FLAMMABLE GAS TANK WASTE LEVEL RECONCILIATION TANK 241-SX-105}

WORK ORDER E40697

Prepared for

Lockheed Martin Hanford Corporation

June 1997

Prepared by

Fluor Daniel Northwest

Richland, Washington 


\section{LETTER REPORT}

\section{FLAMMABLE GAS TANK WASTE LEVEL RECONCILIATION TANK 241-SX-105}

WORK ORDER E40697

Fluor Daniel Northwest
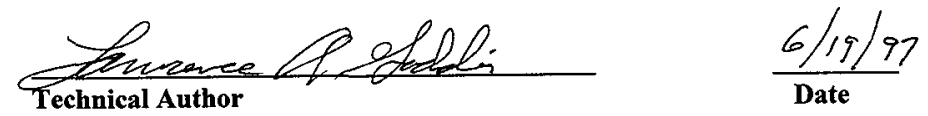

Trobeth Limmermar
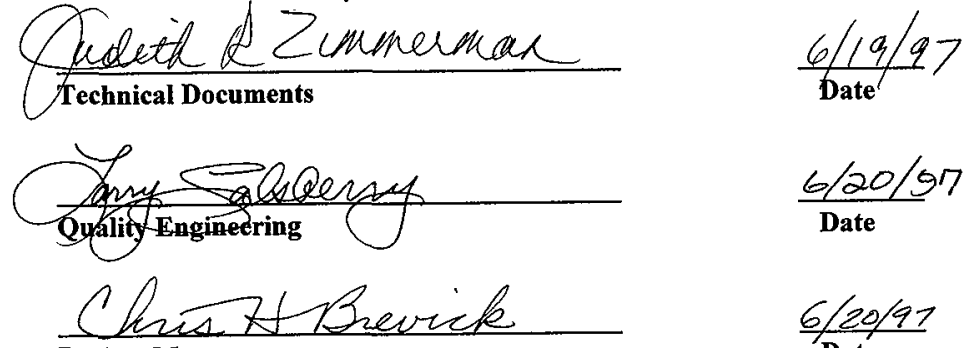

Project Manager

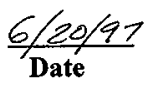

Lockheed Martin Hanford Corporation

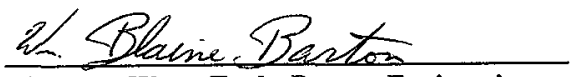

Manager, Waste Tanks Process Engineering

$\frac{6+23-97}{\text { Date }}$


HNF-SD-WM-ER-693, Rev. 0

\section{TABLE OF CONTENTS}

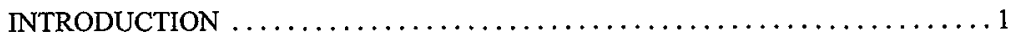

SUMMARY, CONCLUSIONS, AND RECOMMENDATIONS $\ldots \ldots \ldots \ldots \ldots \ldots 2$

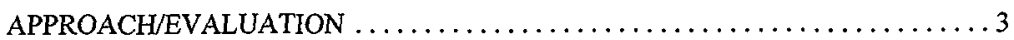

CLARIFICATION OF SCOPE PARAMETERS $\ldots \ldots \ldots \ldots \ldots \ldots 3$

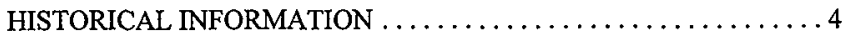

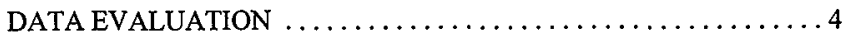

1973 - 1975 Data ...............................

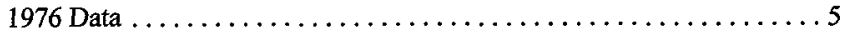

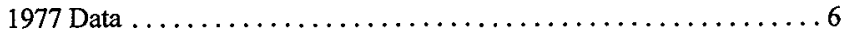

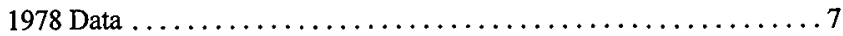

1979 Data ...................................

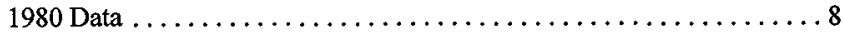

Data Conclusion .............................. 8

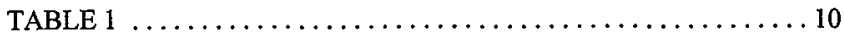

UNCERTAINTIES AND RECOMMENDATIONS FOR FURTHER STUDY $\ldots \ldots 12$

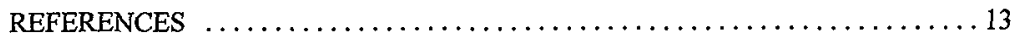

Appendix A. Welty Report Plot and Data

Appendix B. Waste and Level History, 1954 - 1996

Appendix C. Waste Level History Quarterly/Monthly, 1973 - 1980

Appendix D. Waste Level History Plots, June 1973 - December 1980

Appendix E. Waste Level History Data, June 1973 - December 1980

Appendix F. Occurrence Reports, 1973 - 1980 


\section{LETTER REPORT}

\section{FLAMMABLE GAS TANK WASTE LEVEL RECONCILIATION TANK 241-SX-105}

\section{INTRODUCTION}

Fluor Daniel Northwest (FDNW) was authorized to address flammable gas issues by reconciling the unexplained surface level increases in Tank 241-SX-105 (SX-105, typical). The trapped gas evaluation document (ref 1) states that Tank SX-105 exceeds the $25 \%$ of the lower flammable limit (LFL) criterion (ref 2), based on a surface level rise evaluation.

The Waste Storage Tank Status and Leak Detection Criteria document, commonly referred to as the "Welty Report" is the basis for this letter report (ref. 3). The Welty Report is also a part of the trapped gas evaluation document criteria. The Welty Report contains various tank information, including: physical information, status, levels, and dry wells, see Appendix A.

The unexplained waste level rises were attributed to the production and retention of gas in the column of waste corresponding to the unaccounted for surface level rise. From 1973 through 1980, the Welty Report tracked Tank SX-105 transfers and reported a net cumulative change of 20.75 in. This surface level increase is from an unknown source or is unaccounted for. Duke Engineering and Services Hanford (DESH) and Lockheed Martin Hanford Corporation (LMHC) are interested in determining the validity of unexplained surface level changes reported in the Welty Report based upon other corroborative sources of data.

The purpose of this letter report is to assemble detailed surface level and waste addition data from daily tank records, logbooks, and other corroborative data that indicate surface levels, and to reconcile the cumulative unaccounted for surface level changes as shown in the Welty Report from 1973 through 1980. 


\section{SUMMARY, CONCLUSIONS, AND RECOMMENDATIONS}

Tank SX-105 initially received waste from REDOX starting the second quarter of 1955 . After June 1975, the tank primarily received processed waste (slurry) from the 242-S Evaporator/ Crystallizer and transferred supernate waste to Tanks S-102 and SY-102.

The Welty Report shows a cumulative change of 20.75 in. from June 1973 through December 1980. During this period, there were unexplained increases that totaled $25.25 \mathrm{in}$., and decreases that totaled 4.5 in. in the Welty Report. FDNW focused primarily on the larger increases, and after reviewing daily records for the same period, identified an unexplained increase of 52.35 in. and a decrease of 16.05 in., for a net unexplained increase of $36.30 \mathrm{in}$. (see Table 1).

From the FDNW review and comparisons of the Welty Report versus other daily records for Tank SX-105, FDNW determined that the majority of the time, the Welty Report is consistent with daily records. There are slight differences between the Welty Report and the daily tank level records when comparing the larger ( $>1 \mathrm{in}$.) unexplained increases. These differences, could be caused by the method for establish the baseline, by the method used to round off numbers, or by the time that the increase or decrease is identified.

The Welty Report ignored most of the surface level increases (14.10 in. of $18.30 \mathrm{in}$.) when they were identified in Occurrence Reports, 76-40 (+2.2 in.), 76-172 (+3.00 in.), 77-133 (+3.2 in.) and 77-184 (+5.70 in.). In 1978, levels fluctuated considerably more than the Welty Report indicated. In 1979, the Welty Report did not identify any increases or decreases although the levels continued to oscillate. In 1980, the daily records supported the Welty Report.

Because the flammable/trapped gas issue is linked to the unexplained increases in the surface level (ref 1 and 2), FDNW recommends that all occurrence reports concerning tank waste increases or decreases, from 1970 through 1980 , be reevaluated for acceptability of the evaluation as to the cause of the occurrence. This review should include all tank farms to determine if other suspect evaluations have been identified for waste level increases. FDNW also recommends that the Welty 
Report be revised to include the unexplained increases identified by the occurrence reports and that level increases following transfers and increases attributed to flushing be reviewed.

\section{APPROACH/EVALUATION}

\section{CLARIFICATION OF SCOPE PARAMETERS}

The limits of this report were defined based on the Welty Report that exhibit data from June 1973 to July 1987 . Because level data is readily available on PC-SACS, a computer based system with data from 1981 to the present, the scope was limited to 1973 through 1980. The scope was further limited by any complete emptying of the tank between 1973 through 1980 . If it was determined that the tank was emptied, the limit would be from the emptying through 1980.

FDNW evaluated the base line as established by the Welty Report and prepared a plot of that data for Tank SX-105 (see Appendix A). The top portion of this plot shows the increases and decreases of the tank waste level (in inches) versus time as stated in the Welty Report data sheets. The bottom portion of the plot shows the unexplained cumulative changes, time versus inches. As seen in the cumulative change plot, some changes are slow increases and others are rapid changes.

FDNW evaluated Tank S-111 to determine if the tank had been completely emptied during 1973 to 1980. FDNW reviewed the plot of the waste and level history (shown in Appendix B) taken from the historical tank content estimate reports (ref 4, and 5). From this graph, it was determined that there was no evidence of an empty tank during that time. FDNW observed that the plot was generated from quarterly data and felt that monthly level information might reveal additional tank activity not shown by the three-month period.

A plot (shown in Appendix $C$ ) of the tank level was created based on the waste status summary reports (ref 7 through 12). From this level plot, it was determined that Tank S-111 was not emptied during the eight years of concern. Waste summary reports were issued quarterly basis from 1973 
through September 1976, and monthly thereafter. Therefore, quarterly data were used through September 1976 and monthly reports thereafter.

\section{HISTORICAL INFORMATION}

FDNW identified that Tank SX-105 initially received waste from REDOX in the second quarter of 1955. Beginning in June 1975 through September 1977, Tank SX-105 received processed waste (slurry) from the 242-S Evaporator/Crystallizer (242-S Evaporator). Tank SX-105 primarily pumped supernate to Tanks S-101 and S-102, and in April 1977, started pumping to Tanks SY-102, S-107, and S-101. Tank S-102 was the initial feed tank for the 242-S Evaporator, but the feed was changed to Tank SY-102 in the late 1970 s.

The Welty Report identified the occurrence reports for Tank SX-105 that were issued regarding surfaces level changes from 1973 to the end of 1980 , specifically, reports 76-40, 76-172, 77-74, 77-133, 77-184 and 79-125. FDNW retrieved copies of these reports (see Appendix F).

\section{DATA EVALUATION}

FDNW reviewed historic tank farm daily record sheets and tabulated logbook data (ref 13, 14, and 15). From these records, yearly plots of the waste surface level (see Appendix D) and data files were generated (see Appendix E). The plots and data were generated on a monthly basis when the tank was inactive, and on a daily basis when the tank was actively receiving or transferring waste. These plots and data were then compared against the Welty Report plot and data to reconcile the unexplained increases identified in the Welty Report. To reconcile the surface level increases, FDNW looked primarily at the larger $(>1$ in.) unexplained increases or groups of increases.

\section{3 - 1975 Data}

From reviewing the daily transaction details and the daily waste level manual plots and records, FDNW determined that Tank SX-105 was inactive in 1973, 1974, and until May 1975, with a waste/level depth of approximately 344 in. Between May 2 and May 7, 1975, waste was pumped 
from Tank SX-105 to Tank S-102. After the transfer, the waste depth was 137.8 in. For the next eight months, Tank SX-105 actively received slurry from the 242-S Evaporator, and primarily pumped supernate to Tank S-102. The waste depth varied from a low of 63 in. on June 21, 1975, to a high of $362 \mathrm{in}$. during September 1975.

\section{Data}

From January 24 through February 3, 1976, approximately 136 in. of slurry was received from the 242-S Evaporator. Between February 3 and March 31, 1976, there were no identified transfers into the tank while the waste depth increased from 355.45 in. to 361.2 in., or 5.75 in. According to the Welty Report, the first large unexplained surface level increase 5.80 in. occurred April 1, 1976. Between April 1 and April 6, 1976, approximately 150 in. of supernate were pumped to Tank S-102 and approximately 38 in. of slurry were received. From April 6 to April 27, 1976, the waste depth increased by $2.3 \mathrm{in}$., from $249.7 \mathrm{in}$. to $252.0 \mathrm{in}$. (Welty Report, $2.25 \mathrm{in}$ ) with no identified transfers identified on the daily tank farm records (see Table 1).

On April 29 and 30, 1976, Tank SX-105 received additional slurry. This established a new baseline waste depth at 274.2 in. on May 1, 1976. Between May 1 and August 11, 1976, there were no waste transfers except periodic gauge flushing. On August 11, 1976, the tank waste depth was 276.75 in. for an unexplained increase of $2.55 \mathrm{in}$. (Welty Report, $0.35 \mathrm{in}$.). However, Occurrence Report 76-40 identified an increase of $2.1 \mathrm{in}$., from $274.3 \mathrm{in}$. to $276.4 \mathrm{in}$. between, May 1 and June 9, 1976.

On August 12 and 13, 1976, Tank SX-105 received additional slurry. Thereafter, a new baseline 283.1 in. was established. Prior to a transfer to Tank S-102 on November 8, 1976, the waste level had increased to $285.6 \mathrm{in}$., a growth of $2.5 \mathrm{in}$. (Welty Report, $2.5 \mathrm{in}$.).

On November 12 and 13, 1976, a slurry transfer increased the tank waste depth to 355.3 in. Between November 12 and December 15, 1976, the waste level had an unexplained increase of $3.0 \mathrm{in}$. to 358.3 in. (Welty Report, none). This increase was identified in Occurrence Report 76-172. 


\section{Data}

In 1977, the Welty Report identified five separate unexplained waste depth increases: January, 2.8 in.; February, 1.9 in.; April, 2.4 in.; August, 1.9 in.; and November, 1.95 in., for a total of 10.95 in. Waste transfer activity continued similar to 1976 , with slurry receiving and pumping to Tanks S-101, S-102, S-107 and SY-102.

Daily records showed that between December 15, 1976, and January 20,1977, the waste level increased 2.8 in. to 361.1 in. On January 21,1977 , the waste baseline level was established at 350.6 in. after a supernate transfer to Tank S-102. Between January 21 and February 15, 1977, the waste level increased 1.9 in. to a depth of 352.5 in. Tank SX-105 received approximately 6 in. of waste from Tank S-101 on February 16, 1977, for a new baseline of 358.7 in. Between February 16 and April 9, 1977, there were no transfers, but an unexplained increase of 2.4 in. to 361.1 in. was recorded.

Between April 9 and 12, 1977, supernate was pumped from Tank SX-105 to Tank SY-102 establishing a new baseline of 266.4 in. From April 9 to May 15, 1977, the level increased to $267.0 \mathrm{in}$. but then dropped to $265.4 \mathrm{in}$. (-1.0 in.) before receiving slurry on May 27, 1977. The Welty Report showed no decrease and identified Occurrence Report (77-44) 77-74. (NOTE: Error in Welty Report number 77-44, it should be 77-47.)

Slurry was received and supernate transferred to Tanks SY-102 and S-107 from May 29 through June 15,1977 , raising the waste level to 346.3 in. A baseline of 348.5 in. wasn't established until July 5, 1977, after an unexplained increase of $2.2 \mathrm{in}$. (Welty Report, none). There are no recorded transfers into Tank SX-105 during this period. Between July 5 and August 20, 1977, the waste level increased to $353.6 \mathrm{in}$. for an unexplained increase of $5.1 \mathrm{in}$. (Welty Report, $1.9 \mathrm{in}$ ). Occurrence Report 77-133 (Welty Report, 77-13 [error]) identified part of this increase.

NOTE: A comment in 77-133 states "The growth phenomenon is frequently observed in all active SX tanks. Superimposed on this general growth, however, are sudden drops attributed to cracks and crevices in the thick, dry crust." 
From August 20 through September 25, 1977, waste was sent to Tanks S-107 and S-101 and slurry was received from the 242-S Evaporator. The waste level varied from a low of 309.5 in. up to a baseline of 354.6 in. on September 25, 1977. Between September 25 and December 1, 1977, there were no noted transfers in the daily records (ref 13 and 14) while the waste level increased to 362.25 in., for a total increase of 7.65 in. (Welty Report, $1.95 \mathrm{in}$.). Occurrence Report 77-184 identified 4.2 in. of this unexplained increase. Tank waste was pumped to Tank SY-102 from December 1 through 5, 1977, and a new baseline of 275.6 in. was set on December 8, 1977.

\section{Data}

By January 1, 1978, the waste level in Tank SX-105 had increased to 277.3 in., up 1.7 in. from the baseline. During 1978, waste level oscillated with an average depth of 277 in. through September 21, 1978, with no waste transfer activity. The waste level increased to 278.4 in. on January 15,1978 , decreased to 275.7 in. on April 17, 1978, increased to 277.6 in. on June 14, 1978, decreased to $276.8 \mathrm{in}$. on July 14,1978 , increased to $277.6 \mathrm{in}$. on September 1, 1978, increased to $277.5 \mathrm{in}$. on September 20,1978, and then decreased to $275.6 \mathrm{in}$. before a gauge flush on September 22, 1978 that further dropped the level to 275.1 in. (see Table 1). The Welty Report shows an increase of $1.8 \mathrm{in}$. between December 8, 1977, and August 21, 1978, and a 2.20-in. decrease on August 22, 1978, with a FIC gauge flushing. The daily records indicate that the FIC gauge was flushed on September 22, 1978, not August 22, 1978; the waste levels correspond between the Welty Report and the daily records. The waste level increased slightly through the end of the year (see Appendices D and E).

\section{Data}

The daily records show that the waste level baseline (275.6-in.) continued into 1979 with the waste level starting at $276.1 \mathrm{in}$. The level continued to oscillate and slowly increased to $278.2 \mathrm{in}$., between September 22, 1978, and October 1979, an increase of 3.1 in. (Welty Report, none). On October 19, 1979, a transfer from Tank S-107 increased the waste level to 302.55 in. From October 19 to November 6, 1979, the level dropped until a new baseline was established at 299.5 in., an unidentified decrease of 3.05 in. From November 6 through December 14, 1979, levels increased 
to $301.3 \mathrm{in.}$ (an increase of $1.8 \mathrm{in}$ ), and then dropped to $297.2 \mathrm{in}$. (a decrease of $4.1 \mathrm{in}$.) on December 17, 1979. Occurrence Report 79-125 identified part of these level fluctuations. The Welty Report did not identify any activity in 1979.

\section{Data}

On January 17, 1980, a new baseline level of 298.0 in. was established as a result of a recommendations in Occurrence Report 79-125. The waste level increased to $300.0 \mathrm{in}$. (an increase of $2.0 \mathrm{in}$.) by the end of the January 1980, with the possibly of some flush water entering the tank. Waste was transferred to Tank SY-102 and received from Tank S-103 during the first part of February 1980 with a new baseline of 326.6 in. established on February 14, 1980. Levels slowly increased to $328.0 \mathrm{in}$. through May 15, 1980. This 1.4-in. increase was identified in the Welty Report as two separate increases of 0.90 in. and 0.55 in., with the possibility that the 0.55 -in. increase was associated with line flushes. Daily records indicate that some flush water entered the tank but it is difficult to establish an amount.

There was a steady decrease from mid-May 1980 to June 12,1980 , to a level of $326.0 \mathrm{in} .(-2.0 \mathrm{in}$.), followed by a slight increase to $326.5 \mathrm{in}$. (+0.5 in.) in the later part of July 1980 . This equals the 1.3-in. decrease identified in the Welty Report. A saltwell screen was installed August 7, 1980, and waste was transferred to Tank S-107 on August 28, 1980. A baseline of 255.6 in. was set September 2, 1980, and readings fluctuated from $254.75 \mathrm{in}$. to $257.05 \mathrm{in}$. throughout the remainder of 1980 .

\section{Data Conclusion}

During the eight-year evaluation of Tank SX-105, the Welty Report showed a 4.5-in. decrease and a 25.25-in. increase, for a net increase of 20.75-in. cumulative change in Tank SX-105. FDNW addressed the major increases and decreases according to the Welty Report (totaling +25.1 in. and -3.50 in., respectively) and compared these values against the daily records (see Table 1). FDNW also reviewed the occurrences reports from 1973 through 1980 and the period between the time of the transfers and the establishment of the baseline. The FDNW review agrees with the Welty Report 
in general additions (5.80 in. vrs. 5.75 in., 2.25 in. vrs. 2.30 in., etc.). The FDNW evaluation differs from the Welty Report regarding level changes associated with the occurrence reports as follows:

- The Welty Report identified a 0.35-in. increase for August 11, 1976; the total unexplained increase from daily records was $2.55 \mathrm{in}$. The difference of $2.2 \mathrm{in}$. was not accounted for because of the occurrence reports.

- In 1976, the Welty Report identified a 10.90-in. increase while the daily records identified a 16.10-in. increase. The difference of 5.2 in. was not accounted for because of the occurrence reports.

- In 1977 , these differences continue with: 10.95 in. vrs. 22.05 in..

- In 1978, the daily records and the Welty Report increases and decreases balanced out with an increases of $5.4 \mathrm{in}$. vrs. $1.8 \mathrm{in}$. and a decrease of $-5.9 \mathrm{in}$. vrs. $-2.2 \mathrm{in}$., for net of $-0.5 \mathrm{in}$. in the daily records and -0.4 in. in the Welty Report.

The total increases tabulated from daily reports from 1976 through 1980 is $52.35 \mathrm{in}$. versus the Welty Report value of $25.25 \mathrm{in}$. (1973 through 1980), a difference of $26.10 \mathrm{in}$. Most of this difference is accounted for by amounts identified in the occurrence reports (14.1 in.). A 2.2-in. increases occurred between the completion time of a transfer and the time that the baseline was established. The remaining increases resulted from the reporting of the additional cycles. These additional surface level values would also increase the net cumulative change value by $178 \%$, from +20.75 to +37.05 in., thereby, increasing the possibility of additional flammable gas buildup within Tank SX-105. 
TABLE 1

Daily Records versus Welty Report

\begin{tabular}{|c|c|c|l|}
\hline Date & $\begin{array}{c}\text { Daily Records } \\
\text { (in.) }\end{array}$ & $\begin{array}{c}\text { Welty Report } \\
\text { (in.) }\end{array}$ & \multicolumn{1}{|c|}{ Comments } \\
\hline $4 / 1 / 76$ & +5.75 & +5.80 & \\
\hline $4 / 28 / 76$ & +2.30 & +2.25 & \\
\hline $8 / 11 / 76$ & +2.55 & +0.35 & Occurrence Report 76-40 (2.1-in. increase) \\
\hline $11 / 8 / 76$ & +2.50 & +2.50 & \\
\hline $12 / 15 / 76$ & +3.00 & 0 & Occurrence Report 76-172 (3.0-in. increase) \\
\hline $1 / 19 / 77$ & +2.80 & +2.8 & \\
\hline $2 / 15 / 77$ & +1.90 & +1.9 & \\
\hline $4 / 8 / 77$ & +2.40 & +2.4 & \\
\hline $5 / 27 / 77$ & $(-1.0)$ & 0 & Occurrence Report 77-74 (decrease) \\
\hline $7 / 5 / 77$ & +2.20 & 0 & Twenty day delay in establishing baseline. \\
\hline $8 / 20 / 77$ & +5.10 & +1.9 & Occurrence Report 77-133 (increase) \\
\hline $12 / 1 / 77$ & +7.65 & +1.95 & Occurrence Report 77-184 (4.2-in. increase) \\
\hline $1 / 1 / 78$ & +1.7 & 0 & \\
\hline $1 / 15 / 78$ & +1.1 & 0 & \\
\hline $4 / 17 / 78$ & -2.7 & 0 & \\
\hline $6 / 14 / 78$ & +0.9 & 0 & \\
\hline $7 / 14 / 78$ & -0.8 & 0 & \\
\hline $9 / 1 / 78$ & +0.8 & 0 & \\
\hline $9 / 20 / 78$ & +0.9 & +1.8 & Welty Report date (8/20/78) error. \\
\hline $9 / 22 / 78$ & -2.4 & -2.2 & Welty Report date (8/22/78) error. \\
\hline $10 / 13 / 79$ & +3.1 & 0 & \\
\hline $11 / 6 / 79$ & -3.05 & 0 & \\
\hline $12 / 14 / 79$ & +1.8 & 0 & \\
\hline $12 / 17 / 79$ & -4.1 & 0 & Occurrence Report 79-125 (-2.3-in. decrease) \\
\hline
\end{tabular}


TABLE 1 (cont.)

Daily Records versus Welty Report

\begin{tabular}{|c|c|c|c|}
\hline Date & $\begin{array}{c}\text { Daily Records } \\
\text { (in.) }\end{array}$ & $\begin{array}{l}\text { Welty Report } \\
\text { (in.) }\end{array}$ & Comments \\
\hline $1 / 30 / 80$ & +2.0 & & Part of increase may be flush water. \\
\hline $5 / 15 / 80$ & +1.4 & $+0.90,+0.55$ & \\
\hline $6 / 12 / 80$ & -2 & & \\
\hline $7 / 27 / 80$ & +0.5 & -1.3 & \\
\hline $\begin{array}{c}\text { Total } \\
\text { Net }\end{array}$ & $\begin{array}{c}+52.35 /-16.05 \\
+36.30\end{array}$ & $\begin{array}{l}+25.10 /-3.50 \\
+21.6\end{array}$ & $\begin{array}{l}\text { Note: Welty Report totals are } 25.25 \text {-in increase, } \\
4.50 \text {-in decrease, Net } 20.75 \text {-in. Increase. }\end{array}$ \\
\hline
\end{tabular}




\section{UNCERTAINTIES AND RECOMMENDATIONS FOR FURTHER STUDY}

There are questions and concerns regarding some of the results discussed in the data reviewed. A primary concern is the evaluation of Occurrence Reports 76-40, 76-172, 77-74, 77-133, 77-184, and 79-125. These reports explain that, "The surface level increase is evaluated as a build-up of crust and growth characteristic of the salt cake. The concentrated slurry on standing and cooling will start dropping solids out which are less dense than the solution and float to the surface causing the liquid level increase." During telephone discussions, Dan Reynolds (LMHC) and Dan Ferting (Numatec Hanford Corporation) both expressed doubt about this evaluation and with today's waste knowledge, it is felt that the increase was a factor of gas generation. The flammable gas issue has been a concern for a number of years and the probability of gas generation and trapped gas in Tank SX-105 is not that unlikely.

The concern regarding questionable evaluation of identified unexplained increases in tank levels extends to other tanks. FDNW recommends that further studies should look at all tanks in the S and SX Tank Farms, and possibly all tank farms, to determine if the cumulative change value identified in the Welty Report for other tanks may also appear low because of the occurrence reports evaluation.

This letter report primarily looked at daily records for the specific tank being evaluated and briefly looked at daily records for the 242-S Evaporator/Crystallizer. With more review, these records could provide more detail on Evaporator operations and the on amount and types of waste pumped into the tanks. The records also identify the amount flush water used to clean the lines after a transfer. These records could lead to a better understanding of the waste transferred to the tanks and possibly resolve some of the unknown waste additions within the tank.

Records exist on miscellaneous catch tanks and water additions to the tanks. These records were not reviewed during the FDNW evaluation because these additions are normally fairly small and insignificant; however, added together, the additions may resolve some of the concerns in the cumulative level increase. 


\section{REFERENCES}

1. Hodgson, K. M., Anantamula, R. P., Barker, S. A., Fowler, K. D., Hopkins, J. D., Lechelt, J. A., Reynolds, D. A., Hedengren, D. C., Stout, R. E., Winward, R. T., Bingham, J. D., March 1996, Evaluation of Hanford Tanks for Trapped Gas, WHC-SD-WM-ER-526, Rev. 1B, Lockheed Martin Hanford Company, Richland Washington

2. Hopkins, J. D., June 1996, Methodology for Flammable Gas Evaluations, WHC-SD-WM-TI724 Rev. 1, Westinghouse Hanford Company, Richland Washington

3. Welty, R. K., September 1988, Waste Storage Tank Status and Leak Detection Criteria Vol. 1 and 2, WHC-SD-WM-TI-356, Westinghouse Hanford Company, Richland, Washington.

4. Brevick, C. H., Gaddis, L. A., Pickett, W. W., June 1994, Historical Tank Content Estimate for the South West Quadrant of the Hanford 200-West Area, WHC-SD-WM-ER-352, Rev. 0, ICF Kaiser Hanford Company, Richland, Washington.

5. Brevick, C. H., Gaddis, L. A., Johnson, E. D., September 1994, Supporting Document for the Historical Tank Content Estimate for SX Tank Farm, WHC-SD-WM-ER-324, Rev. 0, Westinghouse Hanford Company, Richland, Washington.

6. Brevick, C. H., Gaddis, L. A., Johnson, E. D., September 1994, Supporting Document for the Historical Tank Content Estimate for $S$ Tank Farm, WHC-SD-WM-ER-323, Rev. 0, Westinghouse Hanford Company, Richland, Washington.

7. ARHCO, January - December 1973, Chemical Processing Division Waste Status Summary, Quarterly Reports, ARH-2794 A, B, C and D, Atlantic Richfield Company, Richland, Washington.

8. ARHCO, January - December 1974, Operations Division Waste Status Summary, Quarterly Reports, ARH-CD-133 A, B, C and D, Atlantic Richfield Company, Richland, Washington.

9. ARHCO, January - December 1975 , Production and Waste Management Division Waste Status Summary, Quarterly Reports, ARH-CD-336 A, B, C and D, Atlantic Richfield Company, Richland, Washington.

10. ARHCO, January - September 1976, Production and Waste Management Division Waste Status Summary, Quarterly Reports, ARH-CD-702 A, B and I, Atlantic Richfield Company, Richland, Washington.

11. ARHCO, October 1976- May 1977, Production and Waste Management Division Waste Status Summary, Monthly Reports, ARH-CD-822, Atlantic Richfield Company, Richland, Washington.

12. RHO, June 1977 - December 1980, Waste Status Summary, Monthly Reports, RHO-CD-14, Rockwell Hanford Operations, Richland, Washington. 
13. Tank Farm Liquid Level Readings, 1973 - 1980, Daily Waste Level Values, SX Tank Farm, Records Retention Boxes 58107, 61233, 61236, 68654, 74292, and 74293.

14. Tank Farm Surveillance Waste Level Manual Plots and Records, Retention Boxes 111506, 111507 , and 111509.

15. Brevick, C. H., Gaddis, L. A., Lauhala, D. A., May 1997, Operation Logbook Data, HNF-SDWM-TI-721, Preliminary, Westinghouse Hanford Company, Richland, Washington. 


\title{
APPENDIX A
}

\section{WELTY REPORT PLOT AND DATA}

\author{
PLOT GENERATED BY : R P Schwehr: \\ B. D. Andres
}




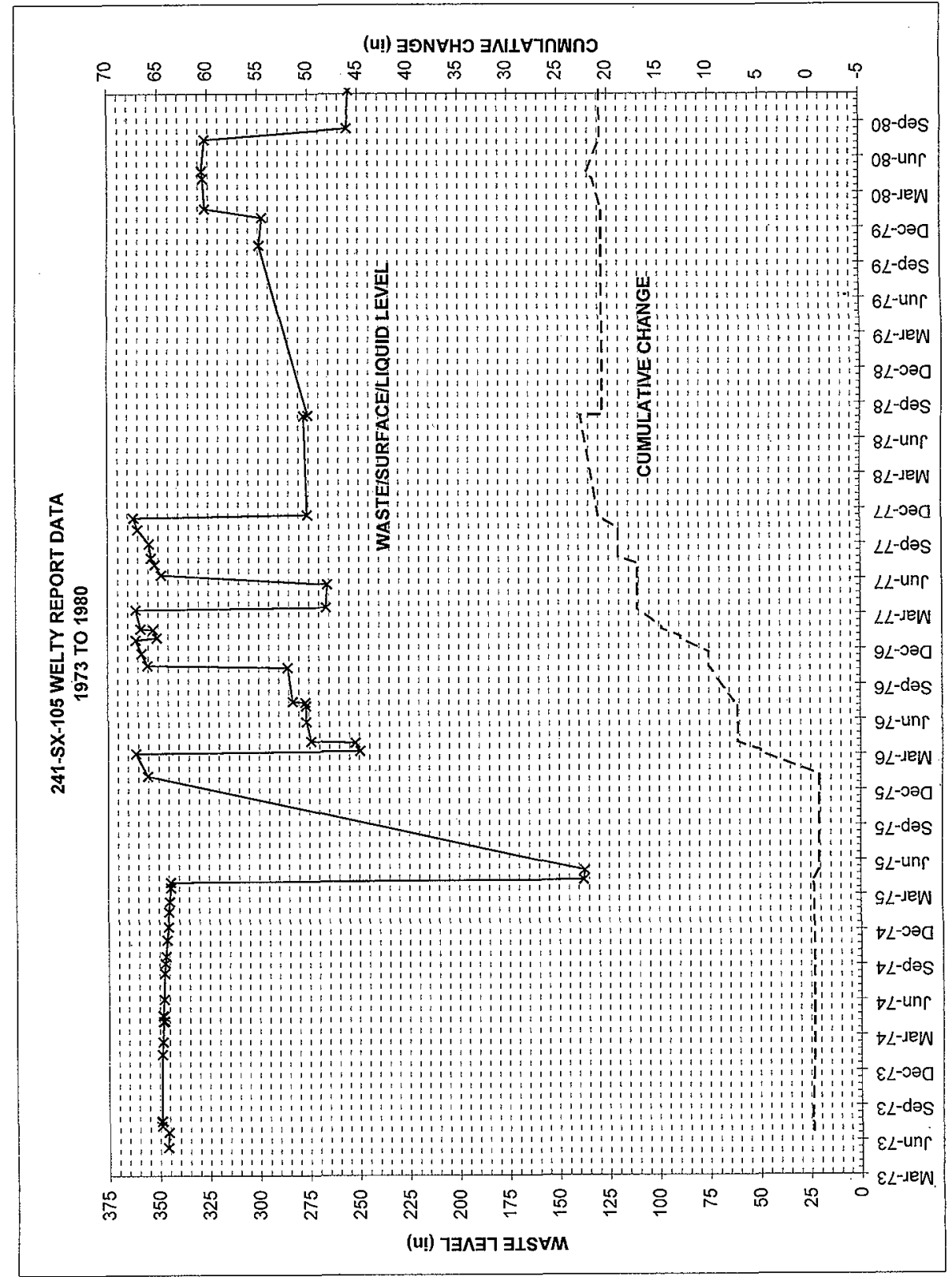


HNF-SD-WM-ER-693, Rev. 0

SO-WM-TI-356

41-05-01

TANK: 105-SX

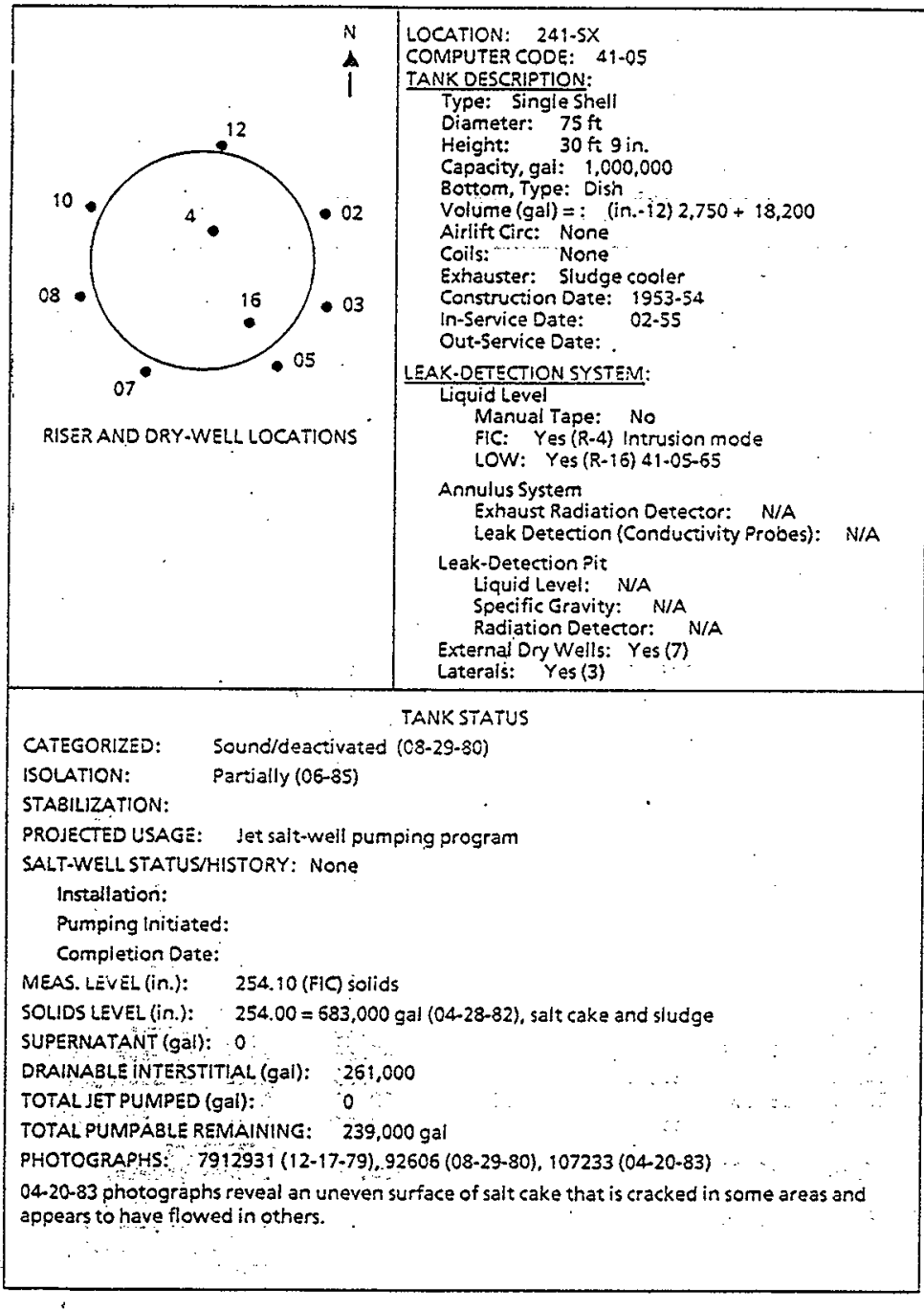

Rey. 0 
HNF-SD-WM-ER-693, Rev. 0

SO-WM-TI-356

41-05-02

TAKK $105-5 X$

Liguto Level.

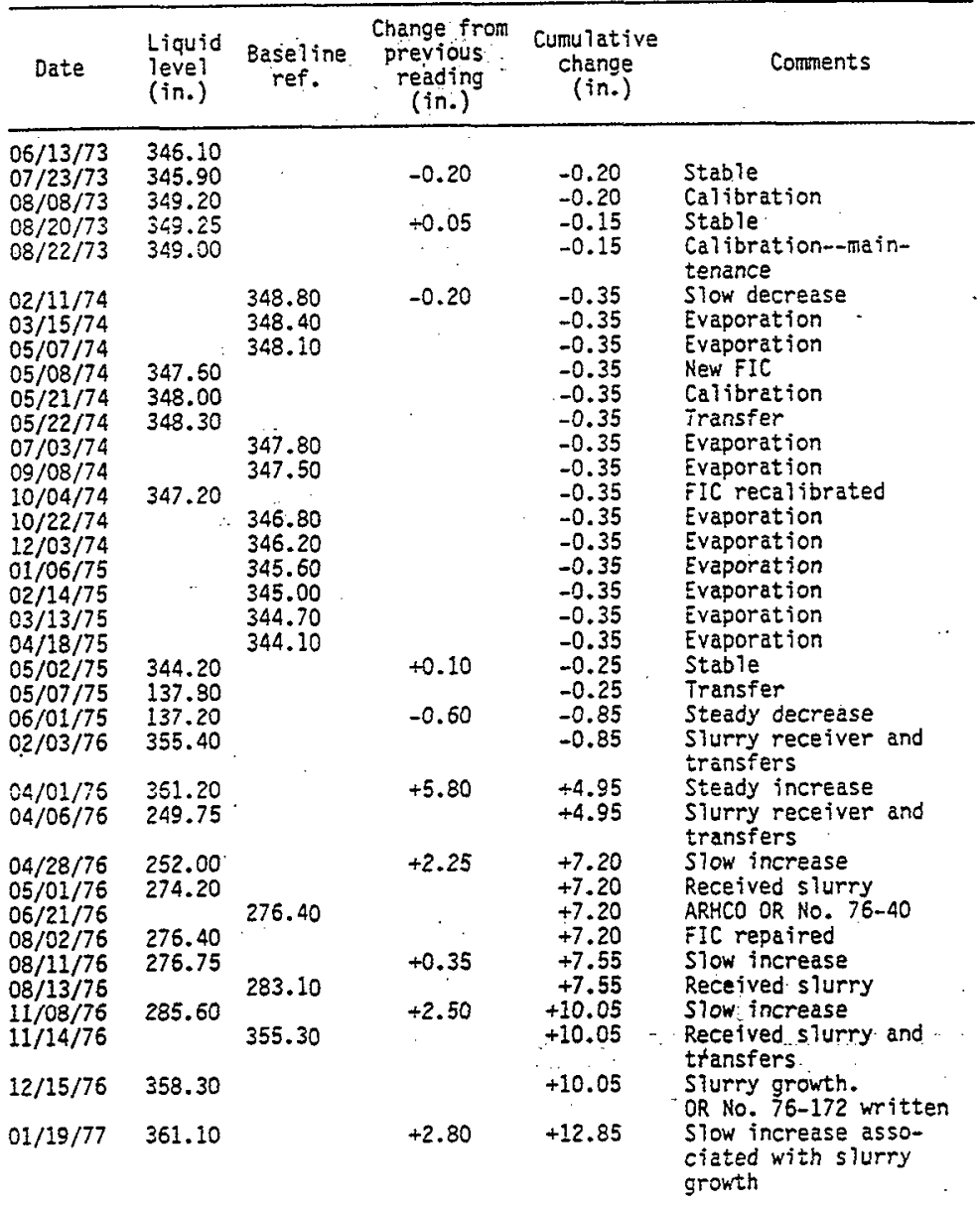

Rev. 0 
HNF-SD-WM-ER-693, Rev. 0

SD-WM-TI-356 41-05-03

TANK 105-5X

Liquid Leve].

\begin{tabular}{|c|c|c|c|c|c|}
\hline Date & $\begin{array}{l}\text { Liquid } \\
\text { level } \\
\text { (in.) }\end{array}$ & $\begin{array}{l}\text { Baseline } \\
\text { ref. }\end{array}$ & $\begin{array}{l}\text { Change from } \\
\text { previous } \\
\text { reading } \\
\text { (in.) }\end{array}$ & $\begin{array}{l}\text { Cumulative } \\
\text { change } \\
\text { (in.) }\end{array}$ & Comments \\
\hline \multirow{4}{*}{$\begin{array}{l}01 / 25 / 77 \\
02 / 15 / 77 \\
02 / 17 / 77 \\
04 / 08 / 77 \\
04 / 13 / 77 \\
05 / 27 / 77\end{array}$} & & \multirow[t]{2}{*}{350.60} & \multirow[b]{2}{*}{+1.90} & \multirow{4}{*}{$\begin{array}{l}+12.85 \\
+14.75 \\
+14.75 \\
+17.15 \\
+17.15 \\
+17.15\end{array}$} & \multirow{4}{*}{$\begin{array}{l}\text { Transfer } \\
\text { Slow increase } \\
\text { Transfer } \\
\text { Slow. increase } \\
\text { Transfer } \\
\text { Decrease. OR No. } 77-44 \\
\text { issued }\end{array}$} \\
\hline & & & & & \\
\hline & 361.10 & \multirow{2}{*}{266.40} & \multirow{2}{*}{+2.40} & & \\
\hline & & & & & \\
\hline $\begin{array}{l}07 / 07 / 77 \\
08 / 03 / 77\end{array}$ & 351.70 & \multirow[t]{2}{*}{348.50} & & $\begin{array}{l}+17.15 \\
+17.15\end{array}$ & $\begin{array}{l}\text { Received siurry } \\
\text { Slurry growth. } \\
\text { OR No. } 77-13\end{array}$ \\
\hline $08 / 20 / 77$ & 353.60 & & \multirow[t]{2}{*}{+1.90} & +19.05 & $\begin{array}{l}\text { Slow increase asso- } \\
\text { ciated with siurry } \\
\text { growth }\end{array}$ \\
\hline $\begin{array}{l}09 / 26 / 77 \\
11 / 01 / 77\end{array}$ & & \multirow[t]{2}{*}{$\begin{array}{l}354.60 \\
360.30\end{array}$} & & $\begin{array}{l}+19.05 \\
+19.05\end{array}$ & \multirow{6}{*}{$\begin{array}{l}\text { growth } \\
\text { Active } \\
\text { Slurry growth. } \\
\text { OR No. } 77-184 \text { written } \\
\text { Steady increase asso- } \\
\text { ciated with slurry } \\
\text { growth } \\
\text { Transfer } \\
\text { Steady increase } \\
\text { Decrease associated } \\
\text { Hith FIC flush } \\
\text { Transfer } \\
\text { OR No. } 79-125 \text { written } \\
\text { Active } \\
\text { Erratic increase } \\
\text { Associated with line } \\
\text { flushes }\end{array}$} \\
\hline $12 / 01 / 77$ & 362.25 & & +1.95 & +21.00 & \\
\hline $12 / 08 / 77$ & & 275.60 & & +21.00 & \\
\hline $\begin{array}{l}08 / 21 / 78 \\
08 / 22 / 78\end{array}$ & $\begin{array}{l}277.40 \\
275.20\end{array}$ & & $\begin{array}{l}+1.80 \\
-2.20\end{array}$ & $\begin{array}{l}+22.80 \\
+20.60\end{array}$ & \\
\hline $\begin{array}{l}11 / 06 / 79 \\
01 / 17 / 80 \\
02 / 09 / 80\end{array}$ & & \multirow[t]{2}{*}{$\begin{array}{l}299.50 \\
298.00 \\
326.60\end{array}$} & & \multirow{2}{*}{$\begin{array}{l}+20.60 \\
+20.60 \\
+20.60 \\
+21.50 \\
+22.05\end{array}$} & \\
\hline $\begin{array}{l}04 / 27 ; 60 \\
05 / 16 / 80\end{array}$ & $\begin{array}{l}327.50 \\
328.05\end{array}$ & & $\begin{array}{l}+0.90 \\
+0.55\end{array}$ & & \\
\hline \multirow[t]{2}{*}{$\begin{array}{l}08 / 05 / 80 \\
09 / 02 / 80 \\
12 / 09 / 80\end{array}$} & 326.65 & \multirow[t]{2}{*}{$\begin{array}{l}255.60 \\
254.50\end{array}$} & \multirow[t]{2}{*}{-1.30} & \multirow[t]{2}{*}{$\begin{array}{l}+20.75 \\
+20.75 \\
+20.75\end{array}$} & \multirow{2}{*}{$\begin{array}{l}\text { Steady decrease. } \\
\text { Active } \\
\text { FIC repaired and } \\
\text { recalibrated. Readings } \\
\text { fluctuate from } 254.75 \\
\text { to } 257.05 \text { in. }\end{array}$} \\
\hline & & & & & \\
\hline $\begin{array}{l}07 / 03 / 81 \\
11 / 18 / 81\end{array}$ & 255.10 & & +0.60 & +21.35 & Erratic increase \\
\hline & & & & & $\begin{array}{l}\text { 10/20/81 readings } \\
\text { fluctuate }\end{array}$ \\
\hline $07 / 28 / 82$ & 255.10 & & +1.30 & +22.65 & Readings fluctuate \\
\hline
\end{tabular}

Rev. 0 
HNF-SD-WM-ER-693, Rev. 0

SO-WM-TI-356

41-05-04

TAKK 105-5X

Liquid Level.

\begin{tabular}{|c|c|c|c|c|c|}
\hline Date. & $\begin{array}{l}\text { Liguid } \\
\text { Tevel } \\
\text { (in.) }\end{array}$ & $\begin{array}{c}\text { Baseline } \\
\text { ref. }\end{array}$ & $\begin{array}{c}\text { Change from } \\
\text { previous } \\
\text { reading } \\
\text { (in.) }\end{array}$ & $\begin{array}{c}\text { Cumulative } \\
\text { change } \\
\text { (in.) }\end{array}$ & Comments \\
\hline $\begin{array}{l}07 / 20 / 83 \\
05 / 21 / 84 \\
05 / 22 / 84\end{array}$ & $\begin{array}{l}254.00 \\
255.00\end{array}$ & 257.00 & $\begin{array}{r}-0.10 \\
+1.00\end{array}$ & $\begin{array}{l}+21.55 \\
+22.55 \\
+22.55\end{array}$ & $\begin{array}{l}\text { Readings fluctuate } \\
\text { Erratic increase } \\
\text { FIC is in intrusion } \\
\text { mode }\end{array}$ \\
\hline $\begin{array}{l}02 / 12 / 85 \\
07 / 01 / 86 \\
07 / 01 / 87\end{array}$ & 254.10 & & & $\begin{array}{l}+22.55 \\
+22.55\end{array}$ & $\begin{array}{l}\text { FIC is in intrusion } \\
\text { mode } \\
\text { Intrusion mode }\end{array}$ \\
\hline
\end{tabular}

Rev. 0 
HNF-SD-WM-ER-693, Rev. 0

SD-WM-TI-356

41-05-10

TANK 105-SX

Photographs on File.

\begin{tabular}{|c|c|c|c|c|c|c|c|c|}
\hline \multirow{2}{*}{$\begin{array}{c}\text { Order } \\
\text { No. }\end{array}$} & \multirow{2}{*}{$\begin{array}{l}\text { Number } \\
\text { of views }\end{array}$} & \multirow{2}{*}{$\begin{array}{l}\text { Photo } \\
\text { date }\end{array}$} & \multicolumn{2}{|c|}{ Synops is } & \multicolumn{2}{|c|}{$\begin{array}{l}\text { Surface-ieve } 1 \\
\text { measurement }\end{array}$} & \multicolumn{2}{|c|}{$\begin{array}{l}\text { Plummet } \\
\text { photo No. }\end{array}$} \\
\hline & & & yes & no & $\begin{array}{c}\text { FIC } \\
\text { (in.) }\end{array}$ & $\begin{array}{c}M T \\
(i n .)\end{array}$ & FIC & $M T$ \\
\hline $\begin{array}{l}753022 \\
754262 \\
756834 \\
752290 \\
770218 \\
774489 \\
7710613 \\
83534 \\
88503 \\
7912931 \\
89152 \\
92606 \\
107233\end{array}$ & $\begin{array}{l}29 \\
36 \\
16 \\
13 \\
13 \\
18 \\
30 \\
31 \\
28 \\
06 \\
09 \\
30 \\
30\end{array}$ & 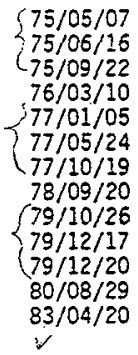 & $\begin{array}{l}x \\
x \\
x\end{array}$ & $\begin{array}{l}x \\
x \\
x \\
x \\
x \\
x \\
x \\
x \\
x \\
x\end{array}$ & $\begin{array}{l}143.30 \\
180.00 \\
353.00 \\
359.50 \\
359.65 \\
255.40 \\
358.60 \\
277.50 \\
300.20 \\
297.30 \\
255.10 \\
253.95\end{array}$ & 299.50 & 1 & 24 \\
\hline
\end{tabular}

Rev. 0 


\section{SUMAARY}

Tank 241-SX-105 is categorized Sound/Deactivated and has been pumped to a minimum supernatant heel. Future plans include installation of a jet pump salt-well system for the removal of as much of the remaining interstitial liquid as is permitted by the existing technology. The tank is connected to the 241-5X sludge cooler facilfty as temperatures in the bulk waste range to above $200^{\circ}$.

New lataral instrumentation was installed and operational during January 1976.

Occurrence Report 79-125 was issued in December 1979 because of a liquid-level decrease. The cause of the observed decrease was attributed to the FIC plumuret contacting surface solids. In May 1984, the FIC was placed into the intrusion mode.

Readings for the dry wells and laterals have remained stable during the review period and are now the only means of leak detection.

\section{ACTION CRITERIA}

1. Dry-welt increase: see p. 41-00-05.

2. Liquid-jevel iimits: see p. 41-00-06.

3. Lateral increase: see p. 41-00-05. 


\section{APPENDIX B}

\section{WASTE AND LEVEL HISTORY $1954-1996$}




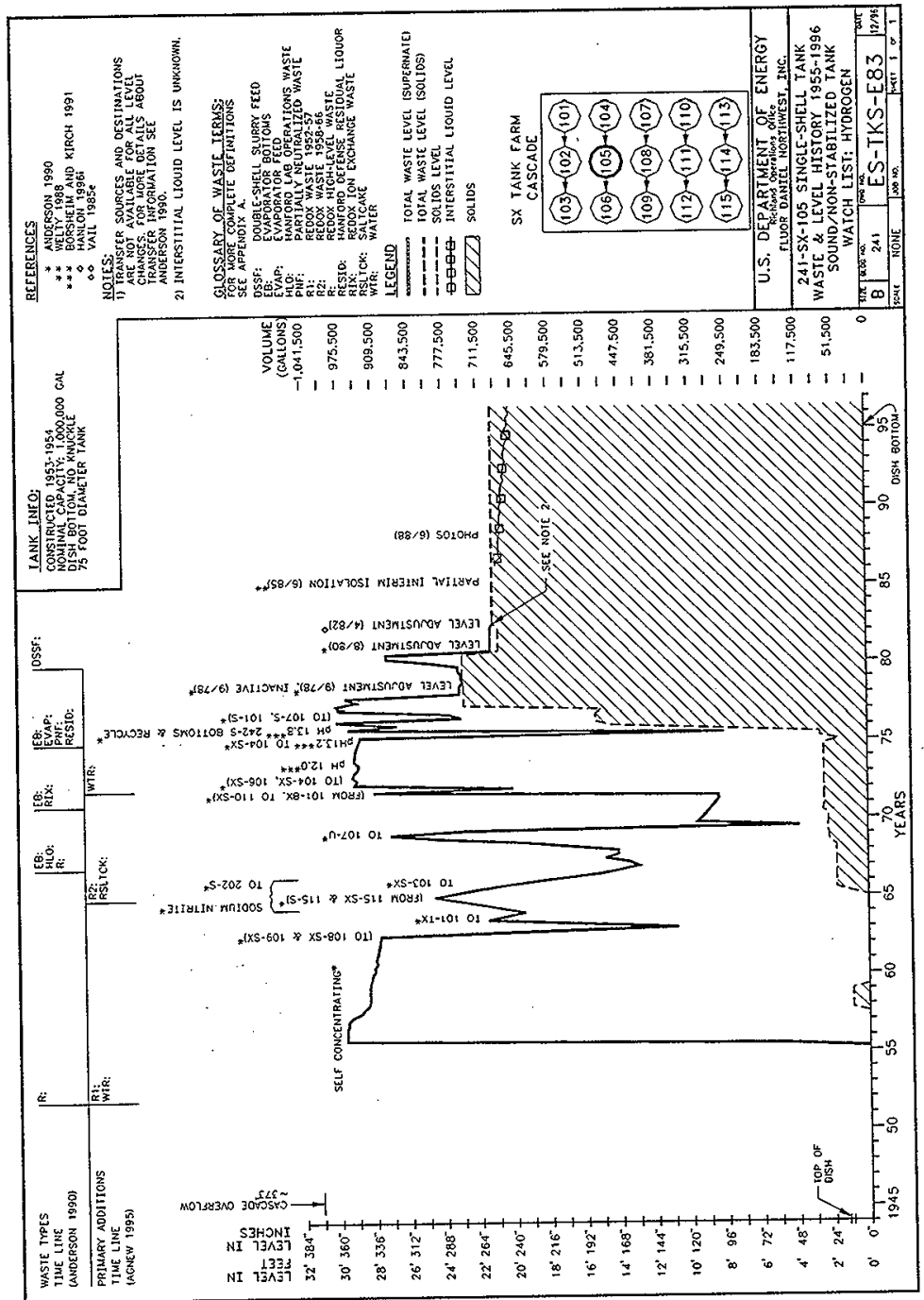


HNF-SD-WM-ER-693, Rev. 0

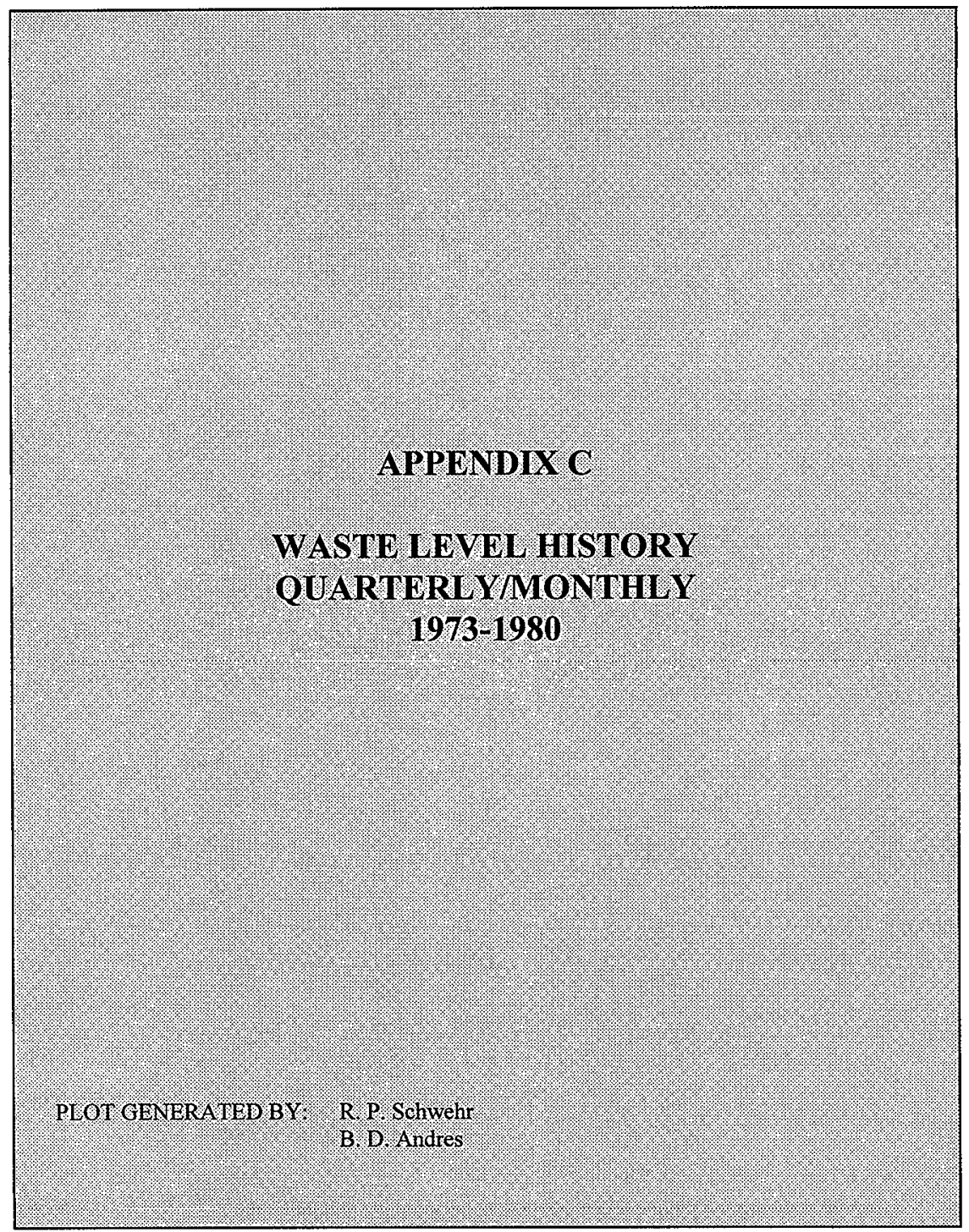




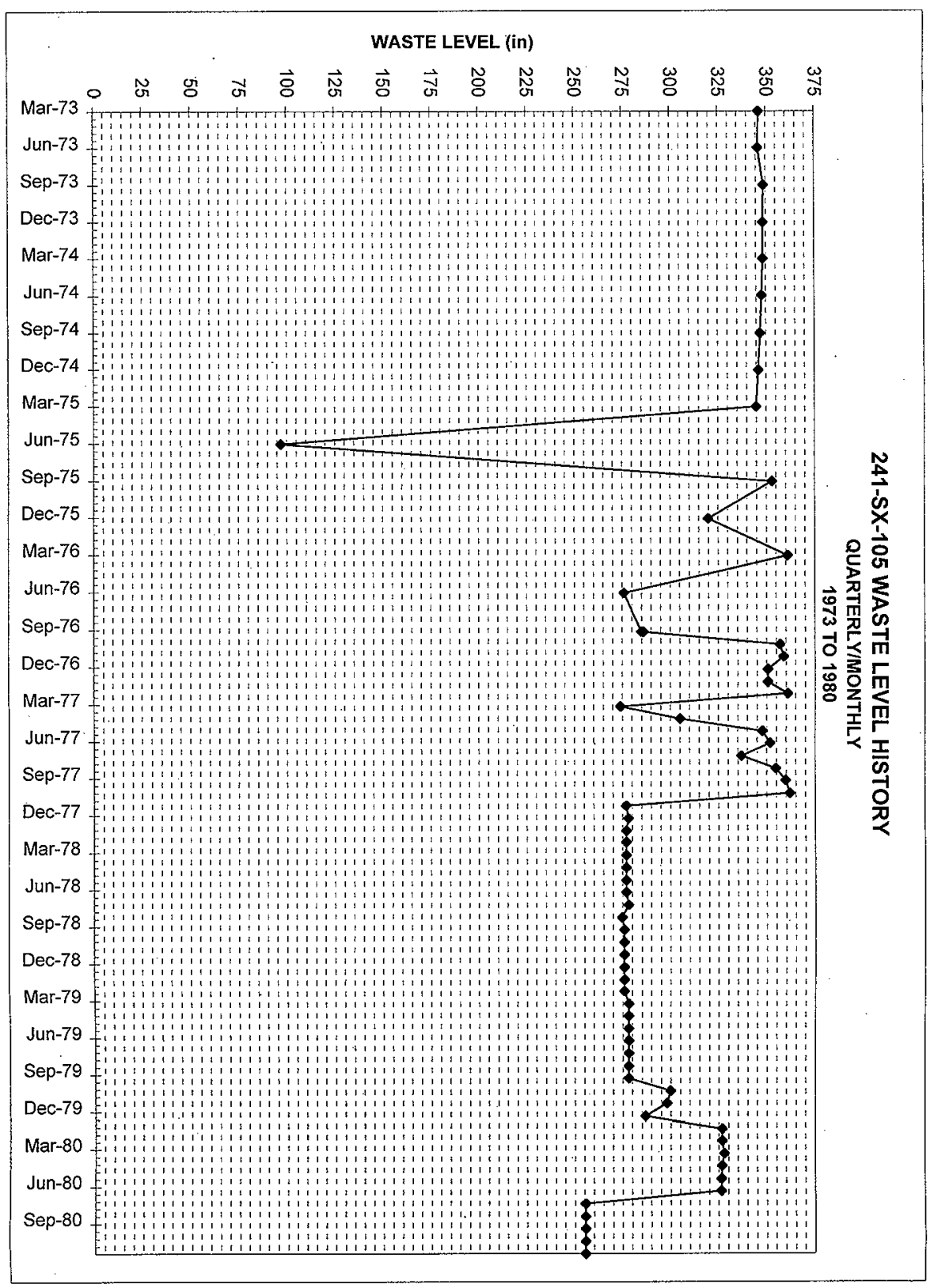


Quarterly/Monthly Waste Volume and Level Summary

241-SX-105

1973 to 1980

\begin{tabular}{|c|c|c|c|c|c|c|c|c|}
\hline $\begin{array}{l}\text { Tank } \\
\text { Number }\end{array}$ & Date & $\begin{array}{l}\text { Liquid } \\
\text { Volume } \\
\text { (kgal) }\end{array}$ & $\begin{array}{c}\text { Sludge } \\
\text { Volume } \\
\text { (includes } \\
\text { saltcake, } \\
\text { kgal) }\end{array}$ & $\begin{array}{c}\text { Total Waste } \\
\text { Volume (kgal) }\end{array}$ & $\begin{array}{c}\text { Total Waste } \\
\text { Level (inches) }\end{array}$ & Comments & Book Number & $\begin{array}{l}\text { Page } \\
\text { Number }\end{array}$ \\
\hline $\mathrm{S} \times 105$ & $3 / 31 / 73$ & 872 & 66 & 938 & 346 & $\begin{array}{l}\text { Evaporator Bottoms Waste-REDOX } \\
\text { Supernatant Ion Exchange Waste }\end{array}$ & ARH-2794 A & 8 \\
\hline $\mathrm{S} \times 105$ & $6 / 30 / 73$ & 871 & 66 & 937 & 346 & $\begin{array}{l}\text { Evaporator Bottoms Waste-REDOX } \\
\text { Supernatant Ion Exchange Waste }\end{array}$ & ARH-2794 B & 8 \\
\hline SX105 & $9 / 30 / 73$ & 879 & 66 & 945 & 349 & $\begin{array}{l}\text { Evaporator Bottoms Waste-REDOX } \\
\text { Supernatant Ion Exchange Waste }\end{array}$ & ARH-2794 C & 8 \\
\hline $5 \times 105$ & $12 / 31 / 73$ & 878 & 66 & 944 & 349 & $\begin{array}{l}\text { Evaporator Bottoms Waste-REDOX } \\
\text { Supernatant Ion Exchange Waste }\end{array}$ & ARH-2794 D & 8 \\
\hline $\mathrm{s} \times 105$ & $3 / 31 / 74$ & 878 & 66 & 944 & 349 & $\begin{array}{l}\text { Evaporator Bottoms Waste-REDOX } \\
\text { Supernatant lon Exchange Waste }\end{array}$ & ARH-CD-133A & 8 \\
\hline $\mathrm{S} \times 105$ & $6 / 30 / 74$ & 876 & 66 & 942 & 348 & $\begin{array}{l}\text { Evaporator Bottoms Waste-REDOX } \\
\text { Supernatant Ion Exchange Waste, } \\
2,000 \text { gal from 111-SX }\end{array}$ & ARH-CD-133B & 8 \\
\hline SX105 & $9 / 30 / 74$ & 874 & 66 & 940 & 347 & $\begin{array}{l}\text { Evaporator Bottoms Waste-REDOX } \\
\text { Supernatant lon Exchange Waste }\end{array}$ & ARH-CD-133C & 8 \\
\hline$S \times 105$ & $12 / 31 / 74$ & 900 & 37 & 937 & 346 & $\begin{array}{l}\text { Evaporator Bottoms Waste-REDOX } \\
\text { Supernatant lon Exchange Waste. } \\
\text { Awaiting solidification. }\end{array}$ & ARH-CD-133D & 8 \\
\hline$s \times 105$ & $3 / 31 / 75$ & 861 & 73 & 934 & 345 & $\begin{array}{l}\text { Evaporator Bottoms Waste-REDOX } \\
\text { Supernatant lon Exchange Waste }\end{array}$ & ARH-CD-336A & 8 \\
\hline $5 \times 105$ & $.6 / 30 / 75$ & 179 & 73 & 252 & 97 & $\begin{array}{l}\text { Evaporator Bottoms Waste. 242-S } \\
\text { bottoms and recycle. Uncertainty in } \\
\text { liquid-to-solid ratio. 206,000 to 104- } \\
\text { SX. }\end{array}$ & ARH-CD-336B & 8 \\
\hline $5 \times 105$ & $9 / 30 / 75$ & 693 & 263 & 956 & 353 & $\begin{array}{l}\text { Evaporator Bottoms Waste. 242-S } \\
\text { bottoms and recycle. Uncertainty in } \\
\text { liquid-to-solid ratio. }\end{array}$ & ARH-CD-336C & 8 \\
\hline$S \times 105$ & $12 / 31 / 75$ & 401 & 464 & 865 & 320 & $\begin{array}{l}\text { Evaporator Bottoms Waste. 242-\$ } \\
\text { bottoms and recycle. Uncertainty in } \\
\text { liquid-to-solid ratio. }\end{array}$ & ARH-CD-336D & 8 \\
\hline $\mathrm{S} \times 105$ & $3 / 31 / 76$ & 487 & 491 & 978 & 361 & $\begin{array}{l}\text { Evaporator Bottoms Waste. } 242-\mathrm{S} \\
\text { bottoms and recycle. Uncertainty in } \\
\text { liquid-to-solid ratio. } 263 \mathrm{kgal} \text { to } 101-\mathrm{S} \text {; } \\
66 \mathrm{kgal} \text { to } 107-\mathrm{S} \text {, this value is not } \\
\text { recorded under } 107-\mathrm{S} \text { entry nor any } \\
\text { other tank entry for same period. }\end{array}$ & ARH-CD-702A & 8 \\
\hline $\mathrm{s} \times 105$ & $6 / 30 / 76$ & 253 & 491 & 744 & 276 & $\begin{array}{l}\text { Evaporator Bottoms Waste. } 242-5 \\
\text { bottoms and recycle. Uncertainty in } \\
\text { liquid-to-solid ratio. }\end{array}$ & ARH-CD-702B & 8 \\
\hline $\mathrm{S} \times 105$ & $9 / 30 / 76$ & 278 & 491 & 769 & 285 & $\begin{array}{l}\text { ACTIVE-EVAPORATOR FEED } \\
\text { (CONCENTRATE) }\end{array}$ & ARH-CD-7021 & 12,33 \\
\hline$S \times 105$ & $10 / 1 / 76$ & 292 & 480 & 772 & 286 & $\begin{array}{l}\text { ACTIVE-EVAPORATOR FEED } \\
\text { (CONCENTRATE) }\end{array}$ & ARH-CD-822 OCT & 13,33 \\
\hline $5 \times 105$ & $11 / 1 / 76$ & 487 & 480 & 967 & 357 & ACTIVE-SPACE-LOWHEAT & ARH-CD-822 NOV & 13,33 \\
\hline SX105 & $12 / 1 / 76$ & 492 & 480 & 972 & 359 & ACTIVE-RESIDUAL LIQUOR & ARH-CD-822 DEC & 15,35 \\
\hline $\mathrm{S} \times 105$ & $1 / 1 / 77$ & 211 & 739 & 950 & 351 & ACTIVE-RESIDUAL LIQUOR & ARH-CD-822 JAN & 15,35 \\
\hline $5 \times 105$ & $2 / 1 / 77$ & 211 & 739 & 950 & 351 & ACTIVE-RESIDUAL LIQUOR & ARH-CD-822 FEB & 15,35 \\
\hline SX105 & $3 / 1 / 77$ & 239 & 739 & 978 & 361 & ACTIVE-RESIDUAL LIQUOR & ARH-CD-822 MAR & 15,35 \\
\hline SX105 & $4 / 1 / 77$ & 0 & 739 & 739 & 274 & ACTIVE-RESIDUAL LIQUOR & ARH-CD-822 APR & 15,35 \\
\hline$s \times 105$ & $5 / 1 / 77$ & 85 & 739 & 824 & 305 & ACTIVE-RESIDUAL LIQUOR & ARH-CD-822 MAY & 15,36 \\
\hline SX105 & $6 / 1 / 77$ & 203 & 739 & 942 & 348 & ACTIVE-RESIDUAL LIQUOR & RHO-CD-14 JUN 77 & 15,36 \\
\hline $.5 \times 105$ & $7 / 1 / 77$ & 214 & 739 & 953 & 352 & ACTIVE-RESIDUAL LIQUOR & RHO-CD-14 JUL 77 & 15,36 \\
\hline$S \times 105$ & $8 / 1 / 77$ & 173 & 739 & 912 & 337 & $\begin{array}{l}\text { ACTIVE-RESIDUAL LIQUOR, } \\
\text { SLURRY RECEIVER }\end{array}$ & RHO-CD-14 AUG 77 & 15,36 \\
\hline
\end{tabular}


Quarterly/Monthly Waste Volume and Level Summary

241-SX-105

1973 to 1980

\begin{tabular}{|c|c|c|c|c|c|c|c|c|}
\hline $\begin{array}{l}\text { Tank } \\
\text { Number }\end{array}$ & Date & $\begin{array}{l}\text { Liquid } \\
\text { volume } \\
\text { (kgal) }\end{array}$ & $\begin{array}{c}\text { Sludge } \\
\text { Volume } \\
\text { (includes } \\
\text { saltcake, } \\
\text { kgal) }\end{array}$ & $\begin{array}{c}\text { Total Waste } \\
\text { Volume (kgal) }\end{array}$ & $\begin{array}{c}\text { Total Waste } \\
\text { Level (inches) }\end{array}$ & Comments & Book Number & $\begin{array}{l}\text { Page } \\
\text { Number }\end{array}$ \\
\hline SX105 & $9 / 1 / 77$ & 222 & 739 & 961 & 355 & $\begin{array}{l}\text { ACTIVE-RESIDUAL LIQUOR, } \\
\text { SLURRY RECEIVER }\end{array}$ & RHO-CD-14 SEP 77 & 15,36 \\
\hline $\mathrm{s} \times 105$ & $10 / 1 / 77$ & 236 & 739 & 975 & 360 & $\begin{array}{l}\text { ACTIVE-RESIDUALL LIQUOR, } \\
\text { SLURRY RECEIVER }\end{array}$ & RHO-CD-14 OCT 77 & 16,38 \\
\hline$s \times 105$ & $11 / 1 / 77$ & 242 & 739 & 981 & 362 & $\begin{array}{l}\text { ACTIVE-RESIDUAL LIQUOR, } \\
\text { SLURRY RECEIVER }\end{array}$ & RHO-CD-14 NOV 77 & 16,38 \\
\hline$S \times 105$ & $12 / 1 / 77$ & 8 & 739 & 747 & 277 & $\begin{array}{l}\text { ACTIVE-RESIDUAL LIQUOR, PART } \\
\text { NEUT FEED }\end{array}$ & RHO-CD-14 DEC 77 & 16,38 \\
\hline $\mathrm{S} \times 105$ & $1 / 1 / 78$ & 11 & 739 & 750 & 278 & $\begin{array}{l}\text { ACTIVE-RESIDUAL LIQUOR, PART } \\
\text { NEUT FEED }\end{array}$ & RHO-CD-14 JAN 78 & 16,38 \\
\hline $\mathrm{s} \times 105$ & $2 / 1 / 78$ & 8 & 739 & 747 & 277 & $\begin{array}{l}\text { ACTIVE-RESIDUAL LIQUOR, PART } \\
\text { NEUT FEED }\end{array}$ & RHO-CD-14 FEB 78 & 16,38 \\
\hline$S \times 105$ & $3 / 1 / 78$ & 8 & 739 & 747 & 277 & $\begin{array}{l}\text { ACTIVE-PARTIAL NEUTRALIZATION } \\
\text { FEED }\end{array}$ & RHO-CD-14 MAR 78 & 16,38 \\
\hline SX105 & $4 / 1 / 78$ & 8 & 739 & 747 & 277 & $\begin{array}{l}\text { ACTIVE-PARTIAL NEUTRALIZATION } \\
\text { FEED }\end{array}$ & RHO-CD-14 APR 78 & 16,38 \\
\hline$. S \times 105$ & $5 / 1 / 78$ & 8 & 739 & 747 & 277 & $\begin{array}{l}\text { ACTIVE-PARTIAL NEUTRALIZATION } \\
\text { FEED }\end{array}$ & RHO-CD-14 MAY 78 & 16,38 \\
\hline SX105 & $6 / 1 / 78$ & 8 & 739 & 747 & 277 & $\begin{array}{l}\text { ACTIVE-PARTIAL NEUTRALIZATION } \\
\text { FEED }\end{array}$ & RHO-CD-14 JUN 78 & 16,39 \\
\hline $\mathrm{s} \times 105$ & $7 / 1 / 78$ & 8 & 739 & 747 & 277 & $\begin{array}{l}\text { ACTIVE-PARTIAL NEUTRALIZATION } \\
\text { FEED }\end{array}$ & RHO-CD-14 JUL 78 & 21 \\
\hline $\mathrm{S} \times 105$ & $8 / 1 / 78$ & 11 & 739 & 750 & 278 & $\begin{array}{l}\text { ACTIVE-PARTIAL NEUTRALIZATION } \\
\text { FEED }\end{array}$ & RHO-CD-14 AUG 78 & 21 \\
\hline$s \times 105$ & $9 / 1 / 78$ & 0 & 741 & 741 & 275 & INACTIVE-PRIMARY STABILIZED & RHO-CD-14 SEP 78 & 21 \\
\hline SX105 & $10 / 1 / 78$ & 3 & 741 & 744 & 276 & INACTIVE-PRIMARY STABBILIZED & RHO-CD-14 OCT 78 & 21 \\
\hline $5 \times 105$ & $11 / 1 / 78$ & 3 & 741 & 744 & 276 & INACTIVE-PRIMARY STABILIZED & RHO-CD-14 NOV 78 & 21 \\
\hline$\$ \times 105$ & $12 / 1 / 78$ & 3 & 741 & 744 & 276 & INACTIVE-PRIMARY STABILIZED & RHO-CD-14 DEC 78 & 21 \\
\hline SX105 & $1 / 1 / 79$ & 3 & 741 & 744 & 276 & INACTIVE-PRIMARY STABILIZED & RHO-CD-14 JAN 79 & 21 \\
\hline$S \times 105$ & $2 / 1 / 79$ & 3 & 741 & 744 & 276 & INACTIVE-PRIMARY STABILIZED & RHO-CD-14 FEB 79 & 21 \\
\hline$S \times 105$ & $3 / 1 / 79$ & 3 & 741 & 744 & 276 & INACTIVE-PRIMARY STABILIZED & RHO-CD-14 MAR 79 & 21 \\
\hline SX105 & $4 / 1 / 79$ & 9 & 741 & 750 & 278 & INACTIVE-PRIMARY STABILIZED & RHO-CD-14 APR 79 & 21 \\
\hline $5 \times 105$ & $5 / 1 / 79$ & 9 & 741 & 750 & 278 & INACTIVE-PRIMARY STABILIZED & RHO-CD-14 MAY 79 & 21 \\
\hline SX105 & $6 / 1 / 79$ & 9 & 741 & 750 & 278 & INACTIVE-PRIMARY STABILIZED & RHO-CD-14 JUN 79 & 21 \\
\hline SX105 & $7 / 1 / 79$ & 9 & 741 & 750 & 278 & INACTIVE-PRIMARY STABILIZED & RHO-CD-14 JUL 79 & 21 \\
\hline $5 \times 105$ & $8 / 1 / 79$ & 9 & 741 & 750 & 278 & INACTIVE-PRIMARY STABILIZED & RHO-CD-14 AUG 79 & 21 \\
\hline $5 \times 105$ & $9 / 1 / 79$ & 9 & 741 & 750 & 278 & INACTIVE-PRIMARY STABILIZED & RHO-CD-14 SEP 79 & 21 \\
\hline $\mathrm{S} \times 105$ & $10 / 1 / 79$ & 9 & 741 & 750 & 278 & $\begin{array}{l}\text { ACTIVE-PARTIAL NEUTRALIZATION } \\
\text { FEED }\end{array}$ & RHO-CD-14 OCT 79 & 21 \\
\hline $5 \times 105$ & $11 / 1 / 79$ & 69 & 741 & 810 & 300 & $\begin{array}{l}\text { ACTIVE-PARTIAL NEUTRALIZATION } \\
\text { FEED }\end{array}$ & RHO-CD-14 NOV 79 & 21 \\
\hline $\mathrm{s} \times 105$ & $12 / 1 / 79$ & 64 & 741 & 805 & 298 & $\begin{array}{l}\text { ACTIVE-PARTIAL NEUTRALIZATION } \\
\text { FEED }\end{array}$ & RHO-CD-14 DEC 79 & 21 \\
\hline$s \times 105$ & $1 / 1 / 80$ & 33 & 741 & 774 & 287 & $\begin{array}{l}\text { ACTIVE-PARTIAL NEUTRALIZATION } \\
\text { FEED }\end{array}$ & RHO-CD-14 JAN 80 & 21 \\
\hline SX105 & $2 / 1 / 80$ & 143 & 741 & 884 & 327 & Active-Double Shell Slurry Feed & RHO-CD-14 FEB 80 & 21 \\
\hline $5 \times 105$ & $3 / 1 / 80$ & 143 & 741 & 884 & 327 & Active-Double Shell Slurry Feed & RHO-CD-14 MAR 80 & 21 \\
\hline SX105 & $4 / 1 / 80$ & 146 & 741 & 887 & 328 & Active-Double Shell Slurry Feed & RHO-CD-14 APR 80 & 21 \\
\hline sX105 & $5 / 1 / 80$ & 143 & 741 & 884 & 327 & Active-Double Shell Slury Feed & RHO-CD-14 MAY 80 & 21 \\
\hline SX105 & $6 / 1 / 80$ & 142 & 741 & 883 & 326 & Active-Double Shell Slurry Feed & RHO-CD-14 JUN 80 & 21 \\
\hline $5 \times 105$ & $7 / 1 / 80$ & 142 & 741 & 883 & 326 & Active-Double Shell Slurry Feed & RHO-CD-14 JUL $80^{\circ}$ & 21 \\
\hline $5 \times 105$ & $8 / 1 / 80$ & 15 & 673 & 688 & 255 & Inactive-Double Shell Slurry Feed & RHO-CD-14 AUG 80 & 21 \\
\hline $\mathrm{S} \times 105$ & $9 / 1 / 80$ & 15 & 673 & 688 & 255 & Inactive-Double Shell Slurry Feed & RHO-CD-14 SEP 80 & 21 \\
\hline $5 \times 105$ & $10 / 1 / 80$ & 15 & 673 & 688 & 255 & Inactive-Double Shell Slurry Feed & RHO-CD-14 OCT 80 & 21 \\
\hline $5 \times 105$ & $11 / 1 / 80$ & 15 & 673 & 688 & 255 & Inactive-Double Shell Slurry Feed & RHO-CD-14 NOV 80 & 21 \\
\hline
\end{tabular}


Quarterly/Monthly Waste Volume and Level Summary

HNF-SD-WM-ER-693, Rev. 0

241-SX-105

1973 to 1980

\begin{tabular}{|c|c|c|c|c|c|c|c|c|}
\hline $\begin{array}{c}\text { Tank } \\
\text { Number }\end{array}$ & Date & $\begin{array}{l}\text { Liquid } \\
\text { Volume } \\
\text { (kgal) }\end{array}$ & $\begin{array}{c}\text { Sludge } \\
\text { Volume } \\
\text { (includes } \\
\text { saltcake, } \\
\text { kgal) }\end{array}$ & $\begin{array}{c}\text { Total Waste } \\
\text { Volume (kgal) }\end{array}$ & $\begin{array}{l}\text { Total Waste } \\
\text { Level (inches) }\end{array}$ & Comments & Book Number & $\begin{array}{l}\text { Page } \\
\text { Number }\end{array}$ \\
\hline SX105 & $12 / 1 / 80$ & 15 & 6.73 & 688 & 255 & Inactive-Double Shell Slumy Feed & RHO-CD-14 DEC 80 & 21 \\
\hline
\end{tabular}




\section{APPENDIX D}

\section{WASTE LEVEL HISTORY PLOTS JUNE 1973 - DECEMBER 1980}

PLOT GENERATIED BY : R. P Schwehr

B. D. Andres: 


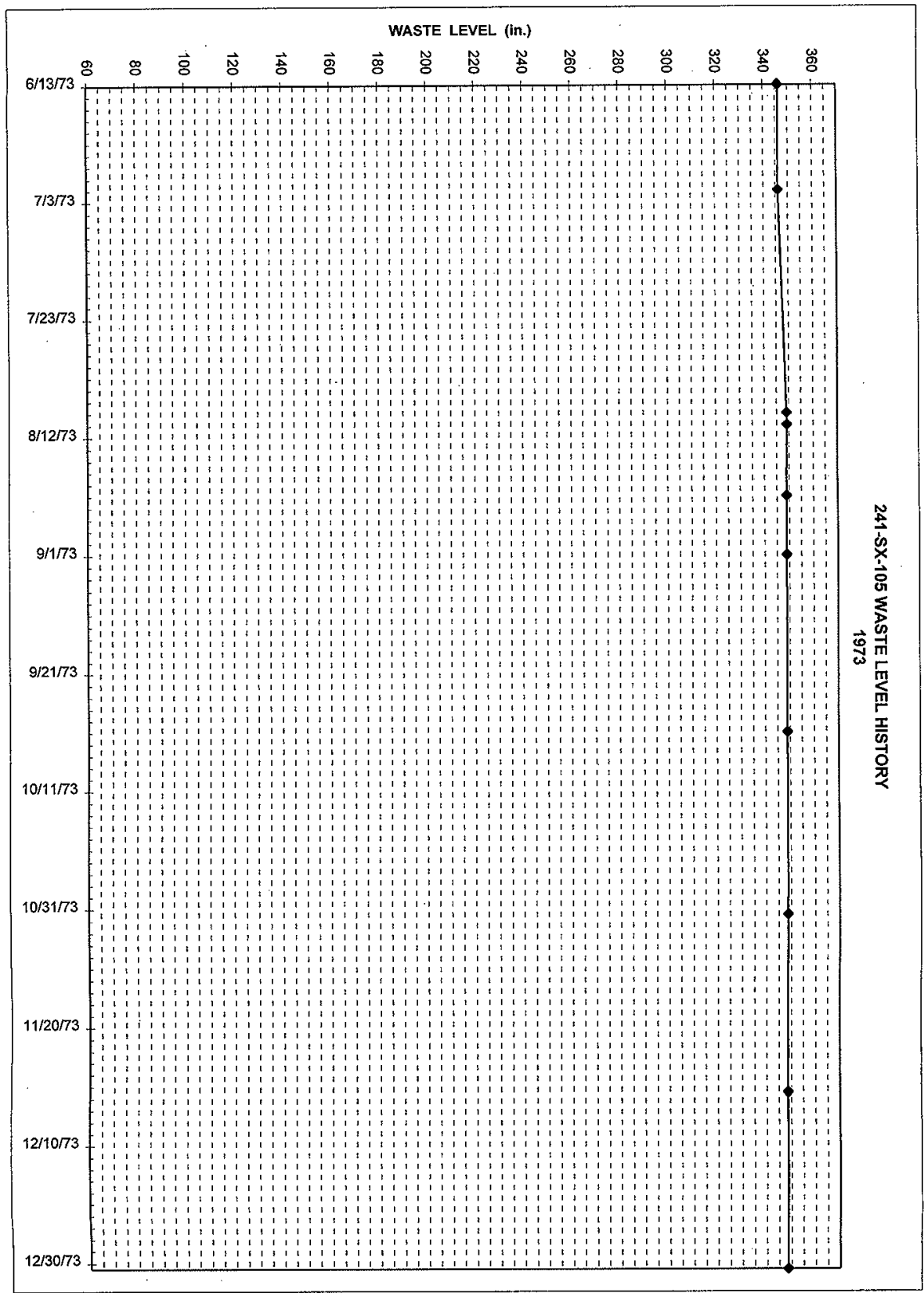

0 ^əу ‘ $869-y \exists-W M-\square S--J N H$ 
HNF-SD-WM-ER-693, Rev. 0

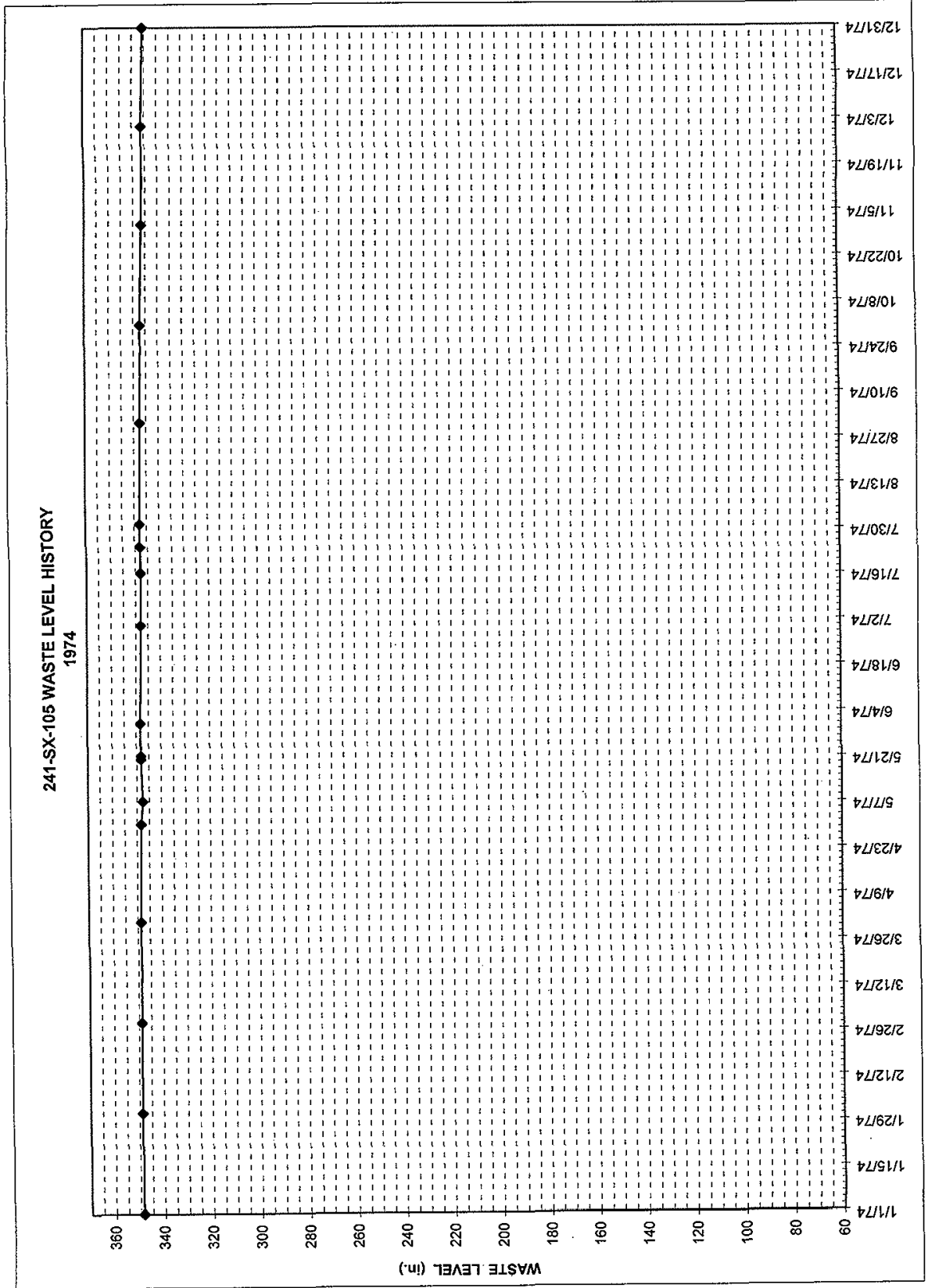




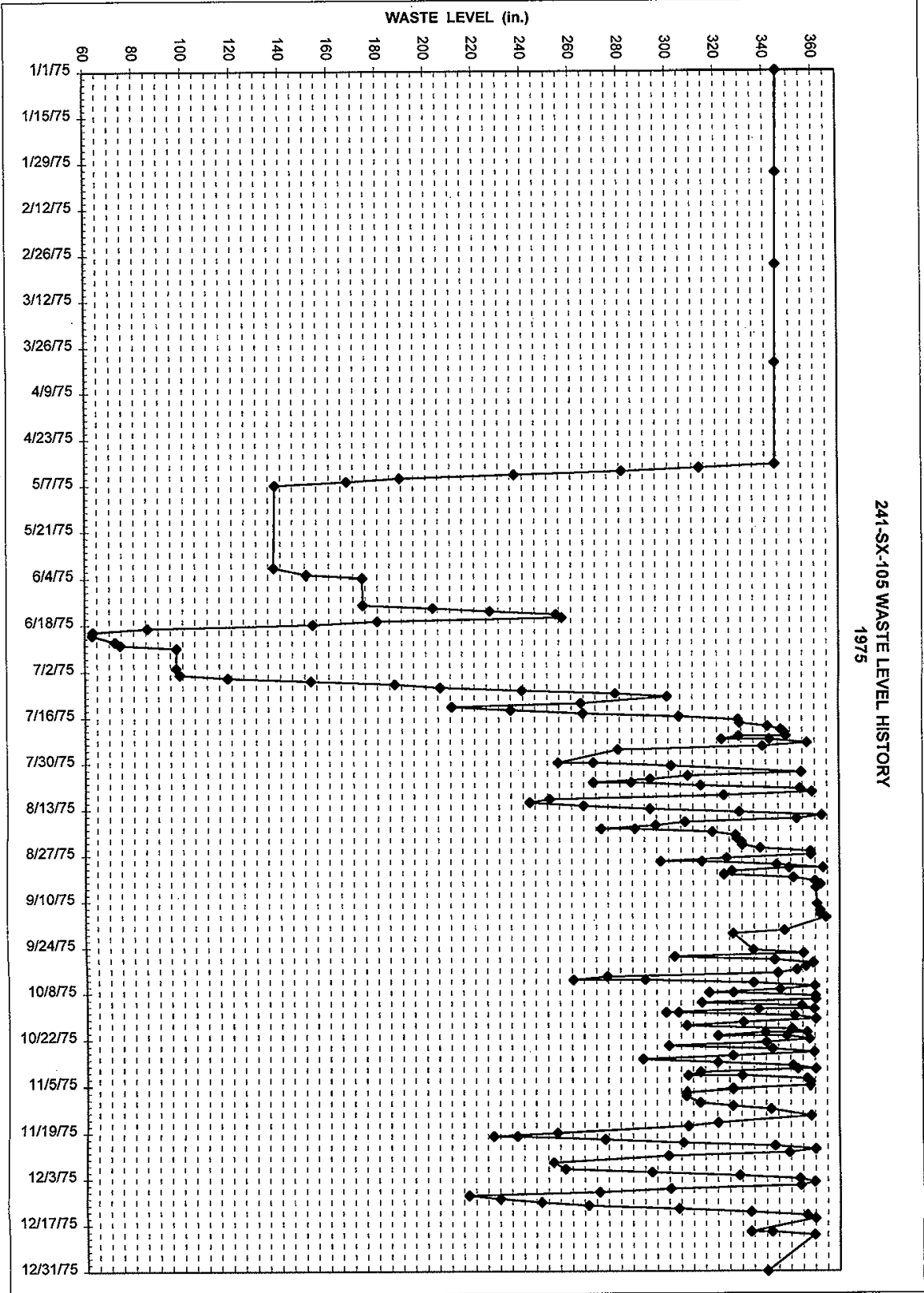




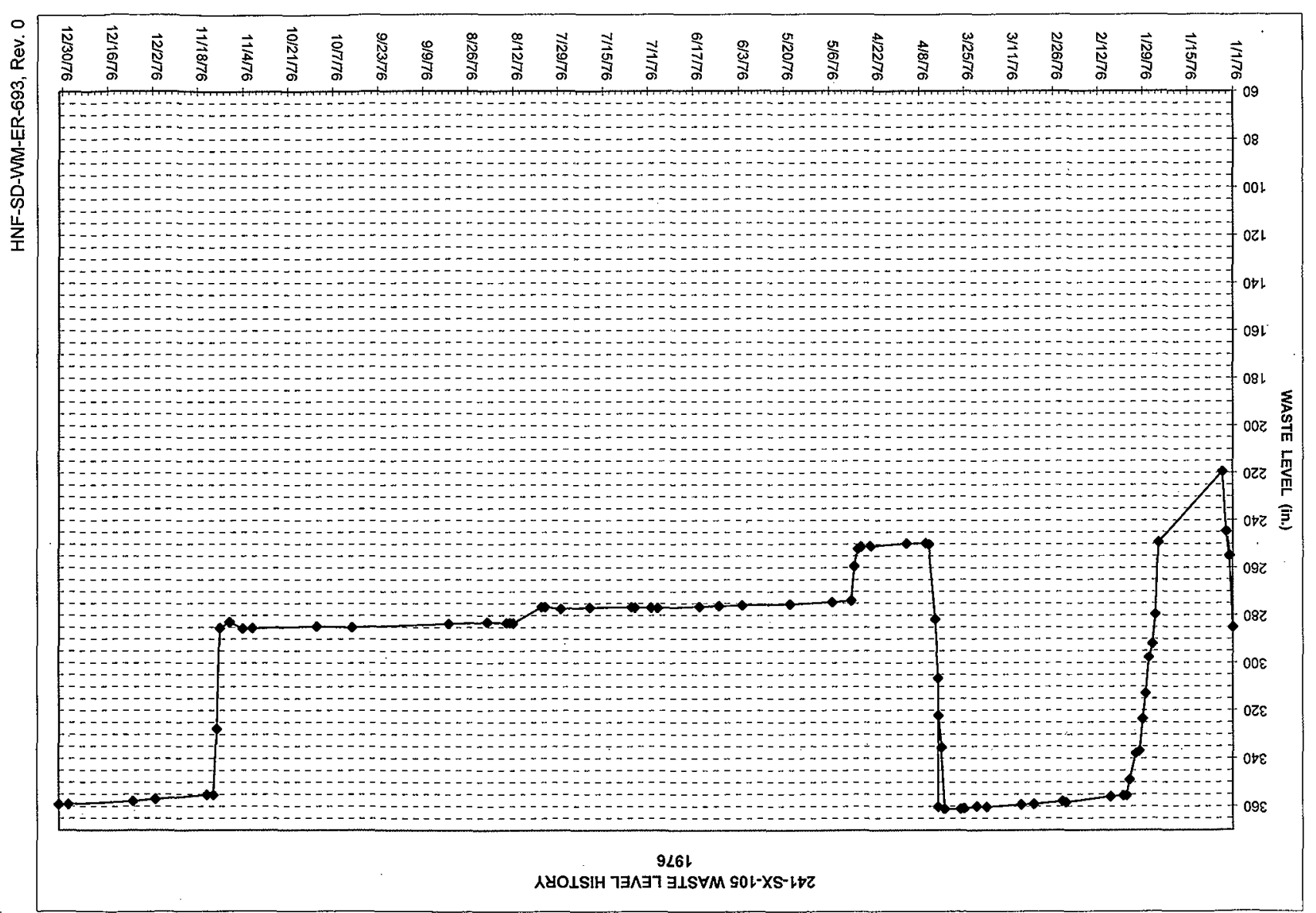




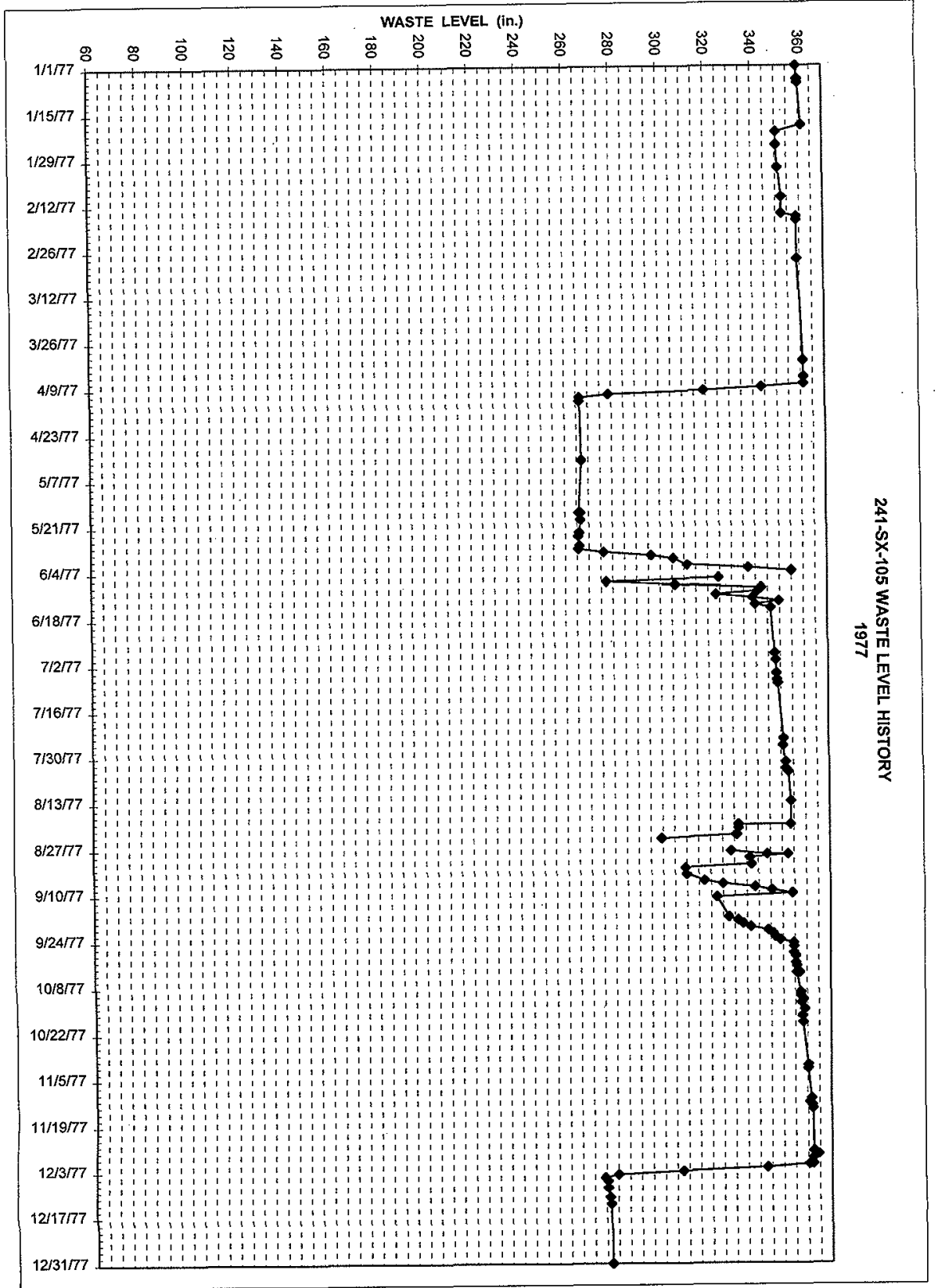




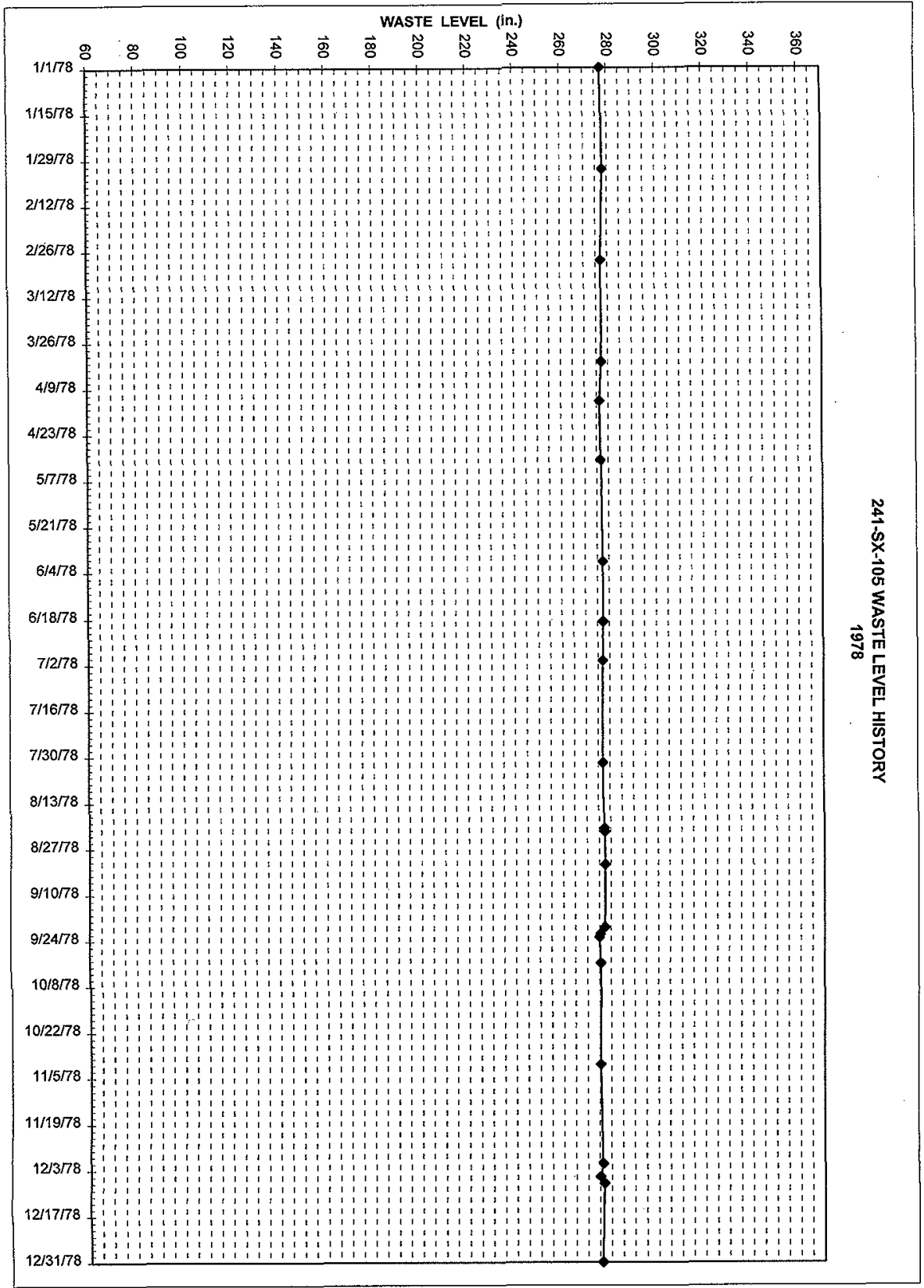

0 ' 


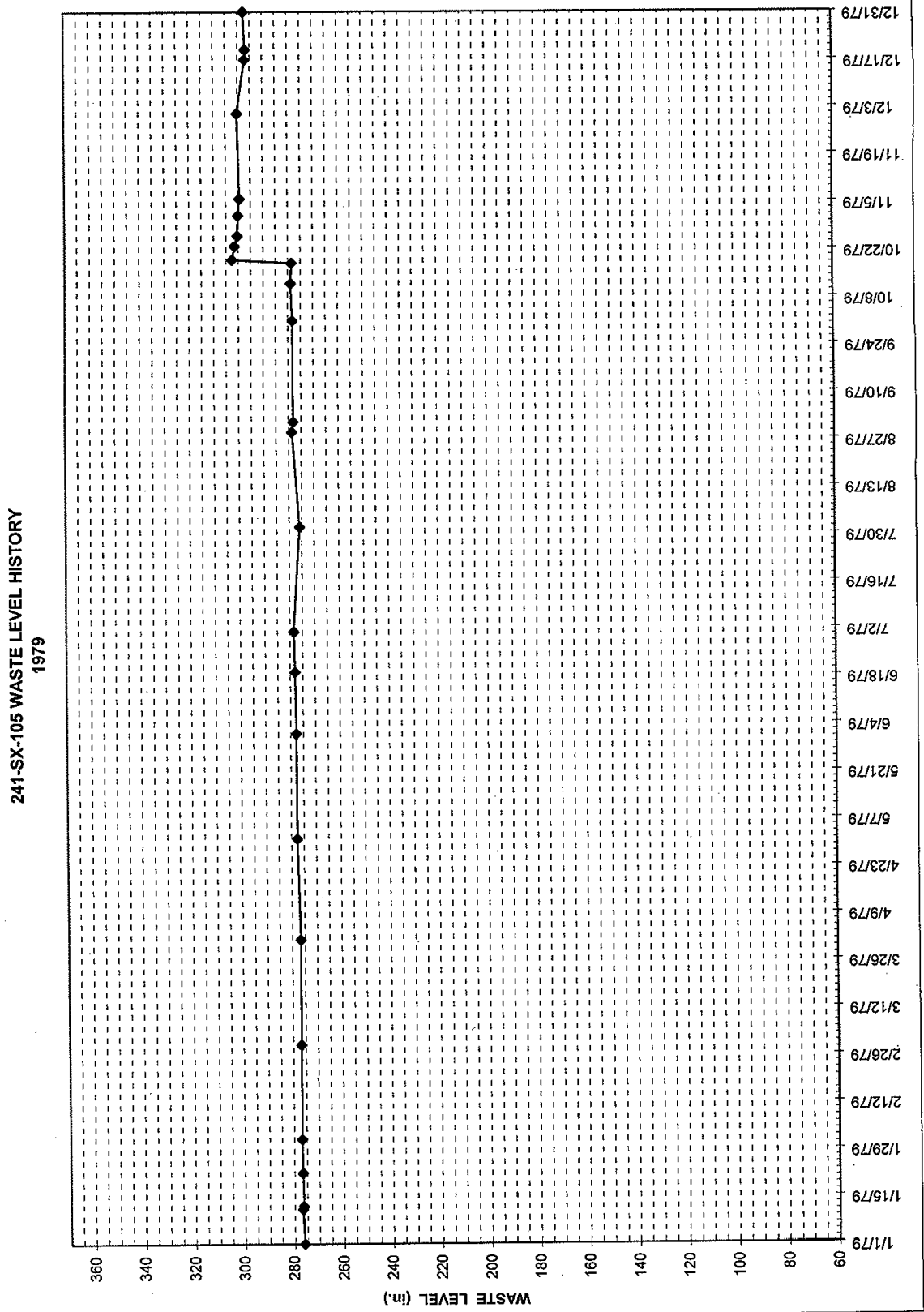

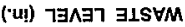


WASTE LEVEL (in.)

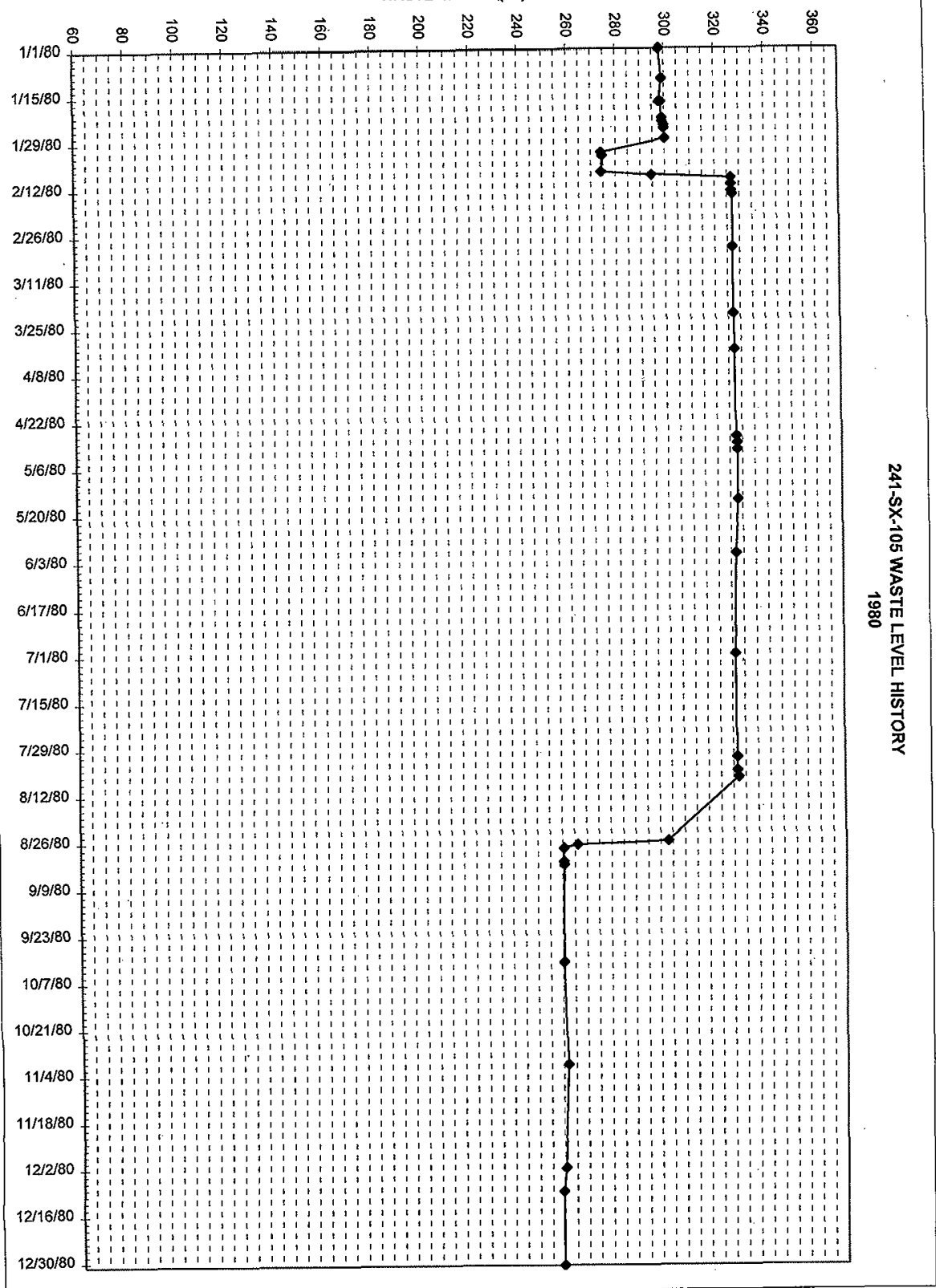

0 ^әу 'ع69-Уヨ-WM-as- $-\mathrm{NH}$ 


\section{APPENDIX E}

\section{WASTE LEVEL HISTORY DATA JUNE 1973 - DECEMBER 1980}

DATA PREPARED BY: : R P Schweht

B. D. Andres 
241-SX-105 Waste Level History

HNF-SD-WM-ER-693, Rev. 0

1973 to 1980

\begin{tabular}{|c|c|c|c|c|c|c|c|c|}
\hline $\begin{array}{l}\text { Tank } \\
\text { Number }\end{array}$ & Date & $\begin{array}{l}\text { Transfer } \\
\text { Source }\end{array}$ & $\begin{array}{c}\text { Transfer } \\
\text { Destination }\end{array}$ & $\begin{array}{c}\text { Tank } \\
\text { Addition } \\
\text { Value } \\
\text { (inches) } \\
\end{array}$ & $\begin{array}{c}\text { Tank } \\
\text { Reduction } \\
\text { Value } \\
\text { (inches) } \\
\end{array}$ & $\begin{array}{l}\text { Waste } \\
\text { Level } \\
\text { Value } \\
\text { (FIC/in) }\end{array}$ & Comments & $\begin{array}{c}\text { Box } \\
\text { Number }\end{array}$ \\
\hline $105 S X$ & $6 / 13 / 73$ & & & & & 346.1 & & 111506 \\
\hline $105 S x$ & $7 / 1 / 73$ & & & & & 346 & & 111506 \\
\hline $105 S X$ & $8 / 8 / 73$ & & & & & 349.15 & & 111506 \\
\hline $105 S X$ & $8 / 10 / 73$ & & & & & 349.25 & & 58107 \\
\hline $105 s x$ & $8 / 22 / 73$ & & & & & 349 & Installed Plastic Liner And Recalibrated & 58107 \\
\hline $105 S x$ & $9 / 1 / 73$ & & & & & 348.9 & & 58107 \\
\hline $105 \mathrm{SX}$ & $10 / 1 / 73$ & & & & & 349 & & 58107 \\
\hline $1055 X$ & $11 / 1 / 73$ & & & & & 348.75 & & 58107 \\
\hline $105 S x$ & $12 / 1 / 73$ & & & & & 348.65 & & 58107 \\
\hline $105 S x$ & $12 / 31 / 73$ & & & & & 348.6 & & 58107 \\
\hline 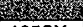 & & & & & & & & \\
\hline $105 \mathrm{~S} x$ & $1 / 1 / 74$ & & & & & 348.6 & & 58107 \\
\hline 105SX & $2 / 1 / 74$ & & & & & 348.8 & & 58107 \\
\hline $105 \mathrm{SX}$ & $3 / 1 / 74$ & & & & & 348.8 & & 58107 \\
\hline $105 S X$ & $4 / 1 / 74$ & & & & & 348.65 & & 58107 \\
\hline $105 \mathrm{SX}$ & $5 / 1 / 74$ & & & & & 348.25 & & 58107 \\
\hline $105 \mathrm{SX}$ & $5 / 8 / 74$ & & & & & 347.6 & New FIC unit installed & 58107 \\
\hline $105 \mathrm{SX}$ & $5 / 21 / 74$ & $111 S X$ & & 0.85 & & 348.25 & & 58107 \\
\hline $105 S X$ & $5 / 22 / 74$ & $111 S X$ & & 0.05 & & 348.3 & Salt well pumping & 58107 \\
\hline 105SX & $6 / 1 / 74$ & & & & & 348.25 & & 58107 \\
\hline $105 S x$ & $7 / 1 / 74$ & & & & & 347.9 & & 58107 \\
\hline $105 S X$ & $7 / \longdiv { 1 7 / 7 4 }$ & & & & & 347.9 & Repaired FIC & 58107 \\
\hline $105 S \mathrm{X}$ & $7 / 2 5 \longdiv { 7 4 }$ & & & & & 347.9 & Installed Foil Safe FIC & 58107 \\
\hline $105 S X$ & $8 \longdiv { 1 / 7 4 }$ & & & & & 347.8 & & 58107 \\
\hline $1055 x$ & $9 / 1 / 74$ & & & & & 347.6 & & 58107 \\
\hline $105 S x$ & $10 / 1 / 74$ & & & & & 347.2 & & 58107 \\
\hline 105SX & $11 / 1 / 74$ & & & & & 346.65 & & 58107 \\
\hline $105 \mathrm{sX}$ & $12 / 1 / 74$ & & & & & 346.2 & & 58107 \\
\hline $1055 x$ & $12 / 31 / 74$ & & & & & 345.7 & & 58107 \\
\hline 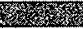 & & & & & & & & 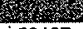 \\
\hline $105 S X$ & $1 / 1 / 75$ & & & & & 345.7 & & 58107 \\
\hline 105SX & $2 / 1 / 75$ & & & & & 345.2 & & 58107 \\
\hline $105 \mathrm{SX}$ & $3 / 1 / 75$ & & & & & $\overline{344.9}$ & & 58107 \\
\hline $1055 x$ & $3 / 31 / 75$ & & & & & 344.45 & & 58107 \\
\hline $105 S \mathrm{X}$ & $5 \longdiv { 1 / 7 5 }$ & & & & & 344.2 & & 61236 \\
\hline $1055 \mathrm{X}$ & $5 / 2 / 75$ & & $102 S$ & & 31 & 313.2 & & 61236 \\
\hline $105 S \mathrm{X}$ & $5 / 3775$ & & & & 32 & 281.2 & Active & 61236 \\
\hline $105 S \mathrm{X}$ & $5 / 4 / 75$ & & 1025 & & 44.4 & 236.8 & & 61236 \\
\hline $1055 X$ & $5 / 5 / 75$ & & & & 47.4 & 189.4 & Active & 61236 \\
\hline $105 \mathrm{SX}$ & $5 / 6 / 75$ & & $102 \mathrm{~S}$ & & 21.9 & 167.5 & & 61236 \\
\hline $105 \mathrm{SX}$ & $5 \longdiv { 7 / 7 5 }$ & & $102 \mathrm{~S}$ & & 29.7 & 137.8 & & 61236 \\
\hline $105 S X$ & $6 / 1 / 75$ & & & & & 137.3 & & 61236 \\
\hline $105 S \mathrm{X}$ & $6 / 3 / 75$ & & & 13.45 & & 150.75 & Receive Slurry & 61236 \\
\hline $105 \mathrm{SX}$ & $6 / 4 / 75$ & & & 23.05 & & 173.8 & Receive Slurry & 61236 \\
\hline 105SX & $6 / 12 / 75$ & & & & & 174.05 & Attempted to pump, flush water added & 61236 \\
\hline $105 \mathrm{SX}$ & $6 / 13 / 75$ & & & 29 & & 203 & Receiving Slurry & 61236 \\
\hline $105 S \mathrm{X}$ & $6 / 14 / 75$ & & & 23.5 & & 226.5 & Active & 61236 \\
\hline $105 S X$ & $6 / 15 / 75$ & & & 27.4 & & 253.9 & Receiving Slurry & 61236 \\
\hline $105 \mathrm{SX}$ & $6 / 16 / 75$ & & & 2.3 & & 256.2 & Receiving Slurry & 61236 \\
\hline $105 S X$ & $6 / 17 / 75$ & & $104 \mathrm{SX}$ & & 76.2 & 180 & & 61236 \\
\hline $105 \mathrm{SX}$ & $6 / 18 / 75$ & & $101 \mathrm{~S}$ & & 26.6 & 153.4 & & 61236 \\
\hline $105 \mathrm{~S} x$ & $6 / 19 / 75$ & & $101 \mathrm{~S}$ & & 67.8 & 85.6 & & 61236 \\
\hline $105 S \mathrm{X}$ & $6 / 20 / 75$ & & $101 S$ & & 22.3 & 63.3 & & 61236 \\
\hline $105 S X$ & $6 / 21 / 75$ & & 1015 & & 0.3 & 63 & & 61236 \\
\hline
\end{tabular}


241-SX-105 Waste Level History 1973 to 1980

\begin{tabular}{|c|c|c|c|c|c|c|c|c|}
\hline $\begin{array}{c}\text { Tank } \\
\text { Number }\end{array}$ & Date & $\begin{array}{l}\text { Transfer } \\
\text { Source }\end{array}$ & $\begin{array}{c}\text { Transfer } \\
\text { Destination }\end{array}$ & $\begin{array}{c}\text { Tank } \\
\text { Addition } \\
\text { Value } \\
\text { (inches) }\end{array}$ & $\begin{array}{c}\text { Tank } \\
\text { Reduction } \\
\text { Value } \\
\text { (inches) }\end{array}$ & $\begin{array}{l}\text { Waste } \\
\text { Level } \\
\text { Value } \\
\text { (FIC/in) }\end{array}$ & Comments & $\begin{array}{l}\text { Box } \\
\text { Number }\end{array}$ \\
\hline $105 \mathrm{~S} X$ & $6 / 23 / 75$ & & & 9 & & 72.3 & Receiving slurry & 61236 \\
\hline $105 \mathrm{SX}$ & $6 / 24 / 75$ & & & 2.2 & & 74.5 & Received from crystallizer & 61236 \\
\hline $105 S X$ & $6 / 25 / 75$ & & & 22.95 & & 97.45 & Received from crystallizer & 61236 \\
\hline $1055 x$ & $7 / 1 / 75$ & & & & & 97.3 & & 61236 \\
\hline $105 S X$ & $7 / 3 / 75$ & & & & & 98.7 & Slurry line flushed $-1,000$ gallons & 61236 \\
\hline $105 S X$ & $7 / 4 / 75$ & & & 19.7 & & 118.4 & Active & 61236 \\
\hline $105 S x$ & $7 / 5 / 75$ & & & 34.1 & & 152.5 & Received slurry & 61236 \\
\hline $105 S x$ & $7 / 6 / 75$ & & & 34.6 & & 187.1 & Received slurry & 61236 \\
\hline $105 S x$ & $7 / 7 / 75$ & & & 18.8 & & 205.9 & Active & 61236 \\
\hline $105 S x$ & $7 / 8 / 75$ & & & 33.75 & & 239.65 & Receiving Slurry & 61236 \\
\hline $105 S X$ & $7 / 9 / 75$ & & & 38.55 & & 278.2 & Received slurry & 61236 \\
\hline $105 S X$ & $7 / 10 / 75$ & & & 21.4 & & 299.6 & Received slurry & 61236 \\
\hline $105 S X$ & $7 / 12 \sqrt{75}$ & & $102 S$ & & 35.6 & 264 & & 61236 \\
\hline $105 S x$ & $7 / 13 / 75$ & & & & 53.4 & 210.6 & Active & 61236 \\
\hline $105 S x$ & $7 / 14 / 75$ & & & 24.4 & & 235 & Received slurry & 61236 \\
\hline $105 \mathrm{SX}$ & $7 / 15 / 75$ & & & 29.8 & & 264.8 & Received slurry & 61236 \\
\hline $105 S \mathrm{X}$ & $7 / 16 / 75$ & & & 39.6 & & 304.4 & Received slurry & 61236 \\
\hline $105 S \mathrm{X}$ & $7 / 17 / 75$ & & & 24.4 & & 328.8 & Active & 61236 \\
\hline $105 \mathrm{SX}$ & $7 / 18 / 75$ & & & 0.6 & & 329.4 & Active & 61236 \\
\hline $105 S \mathrm{X}$ & $7 / 19 / 75$ & & . & 11.4 & & 340.8 & Received slurry & 61236 \\
\hline $105 \mathrm{SX}$ & $7 / 20 / 75$ & & & 5.4 & & 346.2 & Active & 61236 \\
\hline $105 S x$ & $7 / 21 / 75$ & & & 1.5 & & 347.7 & Active & 61236 \\
\hline $105 S x$ & $7 / 22 / 75$ & & & 0.6 & & 348.3 & Received slurry & 61236 \\
\hline $105 S X$ & $7 / 22 / 75$ & & $102 S$ & & 19.3 & 329 & & 61236 \\
\hline $105 S \mathrm{X}$ & $7 / 23 / 75$ & & $102 \mathrm{~S}$ & & 7 & 322 & & 61236 \\
\hline $105 S \mathrm{x}$ & $7 / 23 / 75$ & & & 19.5 & & 341.5 & Received slurry & 61236 \\
\hline 105SX & $7 / 24 / 75$ & & & 15.4 & & 356.9 & Received slurry & 61236 \\
\hline 105SX & $7 / 25 / 75$ & & $102 S$ & & 18.1 & 338.8 & & 61236 \\
\hline $105 \mathrm{SX}$ & $7 / 26 / 75$ & & $102 \mathrm{~S}$ & & 59.6 & 279.2 & & 61236 \\
\hline $105 S X$ & $7 / 30 / 75$ & & 1025 & & 26.1 & 254.4 & & 61236 \\
\hline $105 \mathrm{SX}$ & $7 / 30 / 75$ & & & 14.65 & & 269.05 & Received slurry & 61236 \\
\hline $105 \mathrm{SX}$ & $7 / 31 / 75$ & & & 32.05 & & 301.1 & Received slurry & 61236 \\
\hline $105 \mathrm{SX}$ & $8 / 2 / 75$ & & & 53.4 & & 354.5 & Received sturry & 61236 \\
\hline 105SX & $8 / 3 / 75$ & & $102 \mathrm{~S}$ & & 46.6 & 307.9 & & 61236 \\
\hline 105SX & $8 / 4 / 75$ & & 1025 & & 15.5 & 292.4 & & 61236 \\
\hline $105 \mathrm{SX}$ & $8 / 5 / 75$ & & $102 \mathrm{~S}$ & & 23.4 & 269 & & 61236 \\
\hline $105 \mathrm{~S} X$ & $8 / 5 / 75$ & & & 15.65 & & 284.65 & Active & 61236 \\
\hline $105 S x$ & $8 / 6 \longdiv { 7 5 }$ & & & 28.65 & & 313.3 & Received slurry & 61236 \\
\hline $105 S X$ & $8 / 7 / 75$ & & & 40.7 & & 354 & Received slurry & 61236 \\
\hline $105 \mathrm{SX}$ & $8 / 8 / 75$ & & & 4.8 & & 358.8 & Received slurry & 61236 \\
\hline $105 S X$ & $8 / 9 / 75$ & & $102 S$ & & 36.1 & 322.7 & & 61236 \\
\hline $105 S \mathrm{X}$ & $8 / 10 / 75$ & & & & 71.8 & 250.9 & Active & 61236 \\
\hline $105 \mathrm{SX}$ & $8 / 11 / 75$ & & $102 \mathrm{~S}$ & & 8.2 & 242.7 & & 61236 \\
\hline $1055 x$ & $8 / 12 / 75$ & & & 22.2 & & 264.9 & Received slurry & 61236 \\
\hline $105 S x$ & $8 / 13 / 75$ & & & 27.4 & & 292.3 & Received slurry & 61236 \\
\hline $105 S x$ & $8 / 14 / 75$ & & & 36.8 & & 329.1 & Received slurry & 61236 \\
\hline $105 \mathrm{SX}$ & $8 / 15 / 75$ & & & 33.7 & & 362.8 & Received slurry & 61236 \\
\hline $1055 x$ & $8 / 16 / 75$ & & & & 10.3 & 352.5 & $\begin{array}{l}\text { Active, Flushed FiC and lowered it to } \\
361.8^{\prime \prime}\end{array}$ & 61236 \\
\hline $105 S x$ & $8 / 17 / 75$ & & $102 S$ & & 45.7 & 306.8 & & 61236 \\
\hline $105 \mathrm{SX}$ & $8 / 18 / 75$ & & & & 12.2 & 294.6 & Active & 61236 \\
\hline $105 S x$ & $8 / 19 / 75$ & & $102 S$ & & 22.4 & 272.2 & & 61236 \\
\hline $105 S \mathrm{X}$ & $8 / 19 / 75$ & & & 13.8 & & 286 & Received slurry & 61236 \\
\hline $105 \mathrm{SX}$ & $8 / 20 / 75$ & & & 32 & & 318 & Received siurry & 61236 \\
\hline 105SX & $8 / 21 / 75$ & & & 9.6 & & 327.6 & Received slurry & 61236 \\
\hline
\end{tabular}


241-SX-105 Waste Level History

1973 to 1980

\begin{tabular}{|c|c|c|c|c|c|c|c|c|}
\hline $\begin{array}{c}\text { Tank } \\
\text { Number }\end{array}$ & Date & $\begin{array}{l}\text { Transfer } \\
\text { Source }\end{array}$ & $\begin{array}{c}\text { Transfer } \\
\text { Destination }\end{array}$ & $\begin{array}{l}\text { Tank } \\
\text { Addition } \\
\text { Value } \\
\text { (inches) }\end{array}$ & $\begin{array}{c}\text { Tank } \\
\text { Reduction } \\
\text { Value } \\
\text { (inches) }\end{array}$ & $\begin{array}{l}\text { Waste } \\
\text { Level } \\
\text { Value } \\
\text { (FIC/in) }\end{array}$ & Comments & $\begin{array}{l}\text { Box } \\
\text { Number }\end{array}$ \\
\hline $105 \mathrm{SX}$ & $8 / 22 / 75$ & & & 0.4 & & 328 & & 61236 \\
\hline $1055 x$ & $8 / 23 / 75$ & & & 1.9 & & 329.9 & Active & 61236 \\
\hline $105 S x$ & $8 / 24 / 75$ & & & 0.4 & & 330.3 & Active & 61236 \\
\hline $105 S x$ & $8 / 25 / 75$ & & & 7.3 & & 337.6 & Slurry receiver & 61236 \\
\hline $105 S x$ & $8 / 26 / 75$ & & & 20.6 & & 358.2 & Slurry receiver & 61236 \\
\hline $105 S X$ & $8 / 27 / 75$ & & & 0.1 & & 358.3 & Slurry receiver & 61236 \\
\hline $105 \mathrm{SX}$ & $8 / 28 / 75$ & & $102 \mathrm{~S}$ & & 34.45 & 323.85 & & 61236 \\
\hline $105 S x$ & $8 / 29 / 75$ & & $102 \mathrm{~S}$ & & 27.15 & 296.7 & & 61236 \\
\hline $105 \mathrm{SX}$ & $8 / 29 / 75$ & & & 17 & & 313.7 & Slurry receiver & 61236 \\
\hline $105 S X$ & $8 / 30 / 75$ & & & 30.5 & & 344.2 & Slurry receiver & 61236 \\
\hline $105 S X$ & $8 / 31 / 75$ & & & 19 & & 363.2 & Active & 61236 \\
\hline $105 S X$ & $8 / 31 / 75$ & & & & 13.8 & 349.4 & Active & 61236 \\
\hline $105 S \mathrm{X}$ & $9 / 1 / 75$ & & & & 23.4 & 326 & \begin{tabular}{|l} 
Active \\
\end{tabular} & 61236 \\
\hline $105 S X$ & $9 / 2 / 75$ & & $102 S$ & & 3.2 & 322.8 & & 61236 \\
\hline $1055 x$ & $9 / 3 / 75$ & & & 28.3 & & 351.1 & Slurry receiver & 61236 \\
\hline $105 S x$ & $9 / 4 / 75$ & & & 8.8 & & 359.9 & Slurry receiver & 61236 \\
\hline $105 S x$ & $9 / 5 / 75$ & & & 2.25 & & 362.15 & $\begin{array}{l}105 \mathrm{~S} X \text { has dropped since last being } \\
\text { used as a slurry rec. from } 361.6 \text { to } 360.1 \\
\text { on FIC. The tape is the same } 30^{\prime}-3^{\prime \prime}\end{array}$ & 61236 \\
\hline $105 S \mathrm{X}$ & $9 / 6 / 75$ & & & & 1.85 & 360.3 & Flushed FIC 361.4 & 61236 \\
\hline $105 S X$ & $9 / 11 / 75$ & & & & & 360.8 & & 61236 \\
\hline $105 S x$ & $9 / 13 / 75$ & & & & & 362 & $\begin{array}{l}105 \mathrm{SX} \text { FIC was flushed and it went up } \\
1.8^{\prime \prime} \text {. It is sitting on a hard crust and the } \\
\text { water must fill up a hole the bobber is } \\
\text { sitting in. the manual tape goes slack at } \\
30^{\prime}-43 / 4^{\prime \prime} \text {. }\end{array}$ & 61236 \\
\hline 105SX & $9 / 14 / 75$ & & & & & 362 & Flushed before 362.1 after 364.2 & 61236 \\
\hline $105 \mathrm{SX}$ & $9 / 15 / 75$ & & & & & 364.3 & Tried to pump Flushed FIC & 61236 \\
\hline $105 \mathrm{SX}$ & $9 / 19 / 75$ & & $102 S$ & & 16.45 & 347.4 & & 61236 \\
\hline $105 S X$ & $9 / 20 / 75$ & & $102 \mathrm{~S}$ & & 21 & 326.4 & & 61236 \\
\hline 105SX & $9 / 25 / 75$ & & & 7.05 & & 334.75 & Active & 61236 \\
\hline $105 \mathrm{SX}$ & $9 / 26 / 75$ & & & 20.55 & & 355.3 & Receiving slurry & 61236 \\
\hline $105 \mathrm{SX}$ & $9 / 27 / 75$ & & & & 53 & 302.3 & Active & 61236 \\
\hline $105 \mathrm{SX}$ & $9 / 28 / 75$ & & & 41.2 & & 343.5 & Receiving slurry & 61236 \\
\hline $105 S X$ & $9 / 29 / 75$ & & & 15.8 & & 359.3 & Receiving slurry & 61236 \\
\hline $105 S X$ & $9 / 30 / 75$ & & & & 3.1 & 356.2 & & 61236 \\
\hline $105 S X$ & $10 / 1 / 75$ & & & & 3.6 & 352.6 & $\begin{array}{l}\text { Tried to jump } 105 \mathrm{~S} \times \text { to } 102 \mathrm{~S} \text { but it } \\
\text { would not pump }\end{array}$ & 61236 \\
\hline $105 S X$ & $10 / 2 / 75$ & & & & 7.7 & 344.9 & Active & 61236 \\
\hline $105 S X$ & $10 / 3 / 75$ & & 1015 & & 70.35 & 274.55 & & 61236 \\
\hline $105 S X$ & $10 / 4 / 75$ & & & & 14.05 & 260.5 & Active & 61236 \\
\hline $105 S X$ & $10 / 4 / 75$ & & & 29.8 & & 290.3 & Active & 61236 \\
\hline $105 \mathrm{SX}$ & $10 / 5 / 75$ & & & 44.55 & & 334.85 & Receiving sturry & 61236 \\
\hline $1055 x$ & $10 / 6 / 75$ & & & 25.15 & & 360 & Receiving slurry & 61236 \\
\hline $105 \mathrm{SX}$ & $10 / 7 / 75$ & & & & 14.2 & 345.8 & Active & 61236 \\
\hline $105 \mathrm{~S} x$ & $10 / 8 / 75$ & & $101 \mathrm{~S}$ & & 29.1 & 316.7 & & 61236 \\
\hline $105 S \mathrm{X}$ & $10 / 8 / 75$ & & & 9.9 & & 326.6 & Receiving slurry & 61236 \\
\hline $105 S x$ & $10 / 9 / 75$ & & & 33.6 & & 360.2 & Receiving slurry & 61236 \\
\hline $1055 X$ & $10 / 10 / 75$ & & & 0.2 & & 360.4 & Receiving slurry & 61236 \\
\hline $105 S x$ & $10 / 11 / 75$ & & $102 S$ & & 46.8 & 313.6 & & 61236 \\
\hline $105 S x$ & $10 / 12 / 75$ & & & 41 & & 354.6 & Active & 61236 \\
\hline $105 S X$ & $10 / 13 / 75$ & & & 5.1 & & 359.7 & Slurry Receiver & 61236 \\
\hline $105 S \mathrm{X}$ & $10 / 13 / 75$ & & $102 S$ & & 22.8 & 336.9 & & 61236 \\
\hline $105 S \mathrm{X}$ & $10 / 14 / 75$ & & $102 S$ & & 38.05 & 298.85 & & 61236 \\
\hline
\end{tabular}


241-SX-105 Waste Level History 1973 to 1980

\begin{tabular}{|c|c|c|c|c|c|c|c|c|}
\hline $\begin{array}{l}\text { Tank } \\
\text { Number }\end{array}$ & Date & $\begin{array}{l}\text { Transfer } \\
\text { Source }\end{array}$ & $\begin{array}{c}\text { Transfer } \\
\text { Destination }\end{array}$ & $\begin{array}{l}\text { Tank } \\
\text { Addition } \\
\text { Value } \\
\text { (inches) }\end{array}$ & $\begin{array}{c}\text { Tank } \\
\text { Reduction } \\
\text { Value } \\
\text { (inches) }\end{array}$ & $\begin{array}{l}\text { Waste } \\
\text { Level } \\
\text { Value } \\
\text { (FIC/in) }\end{array}$ & Comments & $\begin{array}{c}\text { Box } \\
\text { Number }\end{array}$ \\
\hline $105 S x$ & $10 / 14 / 75$ & & & 5.15 & & 304 & Slurry Receiver & 61236 \\
\hline $105 \mathrm{SX}$ & $10 / 15 / 75$ & & & 47.7 & & 351.7 & Slurry Receiver & 61236 \\
\hline $1055 x$ & $10 / 16 / 75$ & & & 8.7 & & 360.4 & Slurry Receiver & 61236 \\
\hline $105 S x$ & $10 / 17 / 75$ & & $101 \mathrm{~S}$ & & 29.65 & 330.75 & & 61236 \\
\hline $105 \mathrm{SX}$ & $10 / 18 / 75$ & & & & 23.35 & 307.4 & To 101S \& 102S & 61236 \\
\hline $105 S x$ & 10/19/75 & & & 43.2 & & 350.6 & Slurry Receiver & 61236 \\
\hline $105 \mathrm{~S} X$ & $10 / 20 / 75$ & & & 6.2 & & 356.8 & Slurry Receiver & 61236 \\
\hline $105 S x$ & $10 / 20 / 75$ & & $101 \mathrm{~S}$ & & 17.2 & 339.6 & & 61236 \\
\hline $105 \mathrm{~S} X$ & $10 / 21 / 75$ & & $102 S$ & & 19.3 & 320.3 & & 61236 \\
\hline $105 \mathrm{~S} X$ & $10 / 21 / 75$ & & & 28.2 & & 348.5 & Slurry Receiver & 61236 \\
\hline 105SX & $10 / 22 / 75$ & & & 9.2 & & 357.7 & Slurry Receiver & 61236 \\
\hline $105 S x$ & $10 / 23 / 75$ & & & & 17.6 & 340.1 & Active & 61236 \\
\hline $105 \mathrm{SX}$ & $10 / 24 / 75$ & & & & 40.2 & 299.9 & Active & 61236 \\
\hline $1055 x$ & $10 / 25 / 75$ & & & 42.6 & & 342.5 & Slurry Receiver & 61236 \\
\hline $105 \mathrm{SX}$ & $10 / 26 / 75$ & & & 17.15 & & 359.65 & Slurry Receiver & 61236 \\
\hline $105 S x$ & $10 / 27 / 75$ & & & & 33.25 & 326.4 & active & 61236 \\
\hline $105 S x$ & $10 / 28 / 75$ & & $102 \mathrm{~S}$ & & 37.1 & 289.3 & & 61236 \\
\hline $105 S X$ & $10 / 29 / 75$ & & & 30.9 & & 320.2 & Active & 61236 \\
\hline $105 S x$ & $10 / 30 / 75$ & & & 30.75 & & 350.95 & Active & 61236 \\
\hline $1055 X$ & $10 / 31 / 75$ & & & 9.45 & & 360.4 & Received slurry & 61236 \\
\hline $1055 X$ & $10 / 31 / 75$ & & $102 \mathrm{~S}$ & & 7.5 & 352.9 & & 61236 \\
\hline $105 S x$ & $11 / 1 / 75$ & & & . & 39.8 & 313.1 & Active & 61236 \\
\hline $105 S X$ & $11 / 2 / 75$ & & & & 5.2 & 307.9 & Active & 61236 \\
\hline $105 S X$ & $11 / 2 / 75$ & & & 22.3 & & 330.2 & Active & 61236 \\
\hline $105 S x$ & $11 / 3 / 75$ & & & 26.6 & & 356.8 & Received slurry & 61236 \\
\hline $105 \mathrm{SX}$ & $11 / 4 / 75$ & & & 1.4 & & 358.2 & Received slurry & 61236 \\
\hline $105 S X$ & $11 / 5 / 75$ & & & & & 358 & & 61236 \\
\hline $105 S X$ & $11 / 6 / 75$ & & $102 S$ & & 31.6 & 326.4 & & 61236 \\
\hline $105 S X$ & $11 / 7 / 75$ & & 1025 & & 19.1 & 307.3 & & 61236 \\
\hline $1055 x$ & $11 / 8 / 75$ & & & & & 307.1 & $\begin{array}{l}\text { Flushed B } 307.4 \text { A } 306.2,105 S \mathrm{X} \\
\text { received slurry line flush }\end{array}$ & 61236 \\
\hline $105 \mathrm{SX}$ & $11 / 10 / 75$ & & & 5.8 & & 312.9 & Active & 61236 \\
\hline $105 \mathrm{SX}$ & $11 / 11 / 75$ & & & 13.4 & & 326.3 & Received slurry & 61236 \\
\hline 105SX & $11 / 12 / 75$ & & & 15.7 & & 342 & Received slurry & 61236 \\
\hline $105 \mathrm{SX}$ & $11 / 14 / 75$ & & & 16.45 & & 358.45 & Received slurry & 61236 \\
\hline $105 \mathrm{SX}$ & $11 / 16 / 75$ & & & & 38.2 & 320.25 & Active & 61236 \\
\hline $105 \mathrm{SX}$ & $11 / 17 / 75$ & & $102 \mathrm{~S}$ & & 12.35 & 307.9 & & 61236 \\
\hline $105 \mathrm{SX}$ & $11 / 19 / 75$ & & $102 S$ & & 54.1 & 253.8 & & 61236 \\
\hline $105 \mathrm{SX}$ & $11 / 20 / 75$ & & $102 S$ & & 26.5 & 227.3 & & 61236 \\
\hline $105 S X$ & $11 / 20 / 75$ & & & 9.8 & & 237.1 & Received slurry & 61236 \\
\hline $105 \mathrm{SX}$ & $11 / 21 / 75$ & & & 36.4 & & 273.5 & Received slurry & 61236 \\
\hline $105 \mathrm{SX}$ & $11 / 22 / 75$ & & & 32.4 & & 305.9 & Received slurry & 61236 \\
\hline 105SX & $11 / 23 / 75$ & & & 37.6 & & 343.5 & Active & 61236 \\
\hline $105 S x$ & $11 / 24 / 75$ & & & 16.6 & & 360.1 & Received slurry & 61236 \\
\hline $105 S x$ & $11 / 25 / 75$ & & & & 10.8 & 349.3 & & 61236 \\
\hline $1055 x$ & $11 / 26 / 75$ & & $102 S$ & & 49.65 & 299.65 & & 61236 \\
\hline $105 S x$ & $11 / 28 / 75$ & & 1025 & & 47.55 & 252.1 & & 61236 \\
\hline $105 \mathrm{SX}$ & $11 / 30 / 75$ & & & 4.9 & & 257 & Active & 61236 \\
\hline $105 S X$ & $12 / 1 / 75$ & & & 35.8 & & 292.8 & Received slurry & 61236 \\
\hline $105 S x$ & $12 / 2 / 75$ & & & 36.1 & & 328.9 & Received slurry & 61236 \\
\hline $105 S x$ & $12 / 3 / 75$ & & & 24.7 & & 353.6 & Received slurry & 61236 \\
\hline $105 S x$ & $12 / 4 / 75$ & & & 6 & & 359.6 & Received slurty & 61236 \\
\hline $105 S X$ & $12 / 5 / 75$ & & & & 5.6 & 354 & Active & 61236 \\
\hline $105 S x$ & $12 / 6 / 75$ & & $102 \mathrm{~S}$ & & 53.5 & 300.5 & & 61236 \\
\hline $105 S X$ & $12 / 7 / 75$ & & $102 S$ & & 29.3 & 271.2 & & 61236 \\
\hline
\end{tabular}


241-SX-105 Waste Level History 1973 to 1980

\begin{tabular}{|c|c|c|c|c|c|c|c|c|}
\hline $\begin{array}{l}\text { Tank } \\
\text { Number }\end{array}$ & Date & $\begin{array}{l}\text { Transfer } \\
\text { Source }\end{array}$ & $\begin{array}{c}\text { Transfer } \\
\text { Destination }\end{array}$ & $\begin{array}{l}\text { Tank } \\
\text { Addition } \\
\text { Value } \\
\text { (inches) }\end{array}$ & $\begin{array}{c}\text { Tank } \\
\text { Reduction } \\
\text { Value } \\
\text { (inches) }\end{array}$ & $\begin{array}{c}\text { Waste } \\
\text { Level } \\
\text { Value } \\
\text { (FIC/in) }\end{array}$ & Comments & $\begin{array}{l}\text { Box } \\
\text { Number }\end{array}$ \\
\hline $105 S X$ & $12 / 8 / 75$ & & 1025 & & 54.2 & 217 & & 61236 \\
\hline $105 S x$ & $12 / 9 / 75$ & & & 13 & & 230 & Received slurry & 61236 \\
\hline $105 S X$ & $12 / 10 / 75$ & & & 17.1 & & 247.1 & Received slurry & 61236 \\
\hline $105 S x$ & $12 / 11 / 75$ & & & 19.5 & & 266.6 & Received slurry & 61236 \\
\hline $105 S x$ & $12 / 12 / 75$ & & & 37.4 & & 304 & Received slurry & 61236 \\
\hline $1055 x$ & $12 / 13 / 75$ & & & 29.6 & & 333.6 & Received slurry & 61236 \\
\hline $105 S x$ & $12 / 14 / 75$ & & & 22.9 & & 356.5 & Active & 61236 \\
\hline $105 S X$ & $12 / 15 / 75$ & & & 3.4 & & 359.9 & Received slurry & 61236 \\
\hline $105 S x$ & $12 / 19 / 75$ & & 1025 & & 26.6 & 333.6 & & 61236 \\
\hline $105 S x$ & $12 / 19 / 75$ & & & 8.6 & & 342.2 & Received slurry & 61236 \\
\hline $105 S X$ & $12 / 20 / 75$ & & & 17.45 & & 359.65 & Received slurry & 61236 \\
\hline $105 S x$ & $12 / 31 / 75$ & & $101 \mathrm{~S}$ & & 20.4 & 340.3 & & 61236 \\
\hline 272,2 & 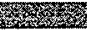 & & 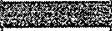 & & & 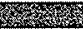 & & \\
\hline $105 S X$ & $1 / 1 / 76$ & & $101 \mathrm{~S}$ & & 55.2 & 285.1 & & 61236 \\
\hline $105 S X$ & $1 / 276$ & & & & 30.1 & 255 & Pumped to $101 \mathrm{~S}$ and $107 \mathrm{~S}$ & 61236 \\
\hline $105 S X$ & $1 / 3 / 76$ & & & & 10.3 & 244.7 & Active & 61236 \\
\hline $105 S X$ & $1 / 4 / 76$ & & $102 \mathrm{~S}$ & & 25.2 & 219.5 & & 61236 \\
\hline $1055 X$ & $1 / 24 / 76$ & & & 28.9 & & 249.3 & Receiving slurry & 61236 \\
\hline $105 S X$ & $1 / 25 / 76$ & & & 30.2 & & 279.5 & Receiving slurry & 61236 \\
\hline $105 \mathrm{SX}$ & $1 / 26 / 76$ & & & 12.5 & & 292 & Receiving slurry & 61236 \\
\hline $105 \mathrm{SX}$ & $1 / 27 / 76$ & & & 5.8 & & 297.8 & Receiving slurry & 61236 \\
\hline $105 S \mathrm{X}$ & $1 / 28 / 76$ & & & 15.1 & & 312.9 & Receiving slurry & 61236 \\
\hline $105 S \mathrm{X}$ & $1 / 29 / 76$ & & & 10.7 & & 323.6 & Receiving slurry & 61236 \\
\hline $105 S X$ & $1 / 30 / 76$ & & & 13.4 & & 337 & Receiving slurry & 61236 \\
\hline $105 S X$ & $1 / 31 / 76$ & & & 0.8 & & 337.8 & Receiving slurry & 61236 \\
\hline $1055 \mathrm{X}$ & $2 / 2 / 76$ & & & 11.2 & & 349 & Receiving slurry & 61236 \\
\hline $105 \mathrm{SX}$ & $2 / 3 / 76$ & & & 6.45 & & 355.45 & Receiving slurry & 61236 \\
\hline $105 S X$ & $2 / 4 / 76$ & & & & & 355.5 & & 61236 \\
\hline $105 S \mathrm{X}$ & $2 / 8 / 76$ & & & & & 356 & Flushed B 356 A 356 & 61236 \\
\hline $105 S \mathrm{X}$ & $2 / 22 / 76$ & & & & & 358.3 & Has been slowly increasing & 61236 \\
\hline $105 S X$ & $2 / 23 / 76$ & & & & & 358 & FLushed B 358.4 A 357.8 & 61236 \\
\hline $105 \mathrm{SX}$ & $3 / 3 / 76$ & & & & & 359 & & 61236 \\
\hline $105 \mathrm{SX}$ & $3 / 7 / 76$ & & & & & 359.3 & Reading has been slowly increasing & 61236 \\
\hline $105 S x$ & $3 / 18 / 76$ & & . & & & 360.3 & $\begin{array}{l}\text { FIC readings have been very erratic } \\
\text { since } 3 / 12776 \text { ranging from } 355.0 \text { to } \\
360.3 \text { changing up to } 5 \text { " in a single shift. } \\
3 / 15 / 76 \text { noted that "Salt cake floating in } \\
\text { tank". }\end{array}$ & 61236 \\
\hline $105 s x$ & $3 / 21 / 76$ & & & & & 360 & $\begin{array}{l}\text { Received slurry } 2 / 3 / 76 \mathrm{LL} 355.4 \text { when } \\
\text { we went out of the TK. FIC reads } 255.0 \\
\text { to } 260.3 \text { in the last few days. }\end{array}$ & 61236 \\
\hline $105 \$ x$ & $3 / 25 / 76$ & & & & & 360.8 & New motor control board & 61236 \\
\hline $105 s x$ & $3 / 26 / 76$ & & & & & 361 & $\begin{array}{l}\text { FIC and Reel tape flushed B } 360.8530^{\prime}- \\
0^{\prime \prime}, A 361.2530^{\prime}-1 / 4^{\prime \prime}\end{array}$ & 61236 \\
\hline $105 S X$ & $3 / 31 / 76$ & & & & & 361.1 & & 61236 \\
\hline $105 S x$ & $4 / 1 / 76$ & & $102 S$ & & 25.5 & 335.5 & & 61236 \\
\hline $105 S x$ & $4 / 2 / 76$ & & $102 S$ & & 13.3 & 322.2 & & 61236 \\
\hline $105 S x$ & $4 / 2 / 76$ & & & 38.1 & & 360.3 & Active & 61236 \\
\hline $105 S x$ & $4 / 2 / 76$ & & $102 \mathrm{~S}$ & & 53.8 & 306.5 & & 61236 \\
\hline $105 S x$ & $4 / 3 / 76$ & & $102 S$ & & 24.8 & 281.7 & & 61236 \\
\hline $105 S \mathrm{X}$ & $4 / 5 / 76$ & & $102 \mathrm{~S}$ & & 31.5 & 250.2 & & 61236 \\
\hline $105 S X$ & $4 / 6 / 76$ & & $102 S$ & & 0.5 & 249.7 & & 61236 \\
\hline $105 S X$ & $4 / 12 / 76$ & & & & & 249.8 & New motor in the FIC & 61236 \\
\hline $1058 X$ & $4 / 23 / 76$ & & & & & 251 & Flushed B 251.5 A 251.15 & 61236 \\
\hline $105 S X$ & $4 / 26 / 76$ & & & & & 251.2 & Flushed tape B $20^{\prime} 101 / 2^{\prime \prime}$ A $20^{\prime} 10^{\prime \prime}$ & 61236 \\
\hline
\end{tabular}


241-SX-105 Waste Level History

HNF-SD-WM-ER-693, Rev. 0

1973 to 1980

\begin{tabular}{|c|c|c|c|c|c|c|c|c|}
\hline $\begin{array}{l}\text { Tank } \\
\text { Number }\end{array}$ & Date & $\begin{array}{l}\text { Transfer } \\
\text { Source }\end{array}$ & $\begin{array}{c}\text { Transfer } \\
\text { Destination }\end{array}$ & $\begin{array}{c}\text { Tank } \\
\text { Addition } \\
\text { Value } \\
\text { (inches) }\end{array}$ & $\begin{array}{c}\text { Tank } \\
\text { Reduction } \\
\text { Value } \\
\text { (inches) }\end{array}$ & $\begin{array}{l}\text { Waste } \\
\text { Level } \\
\text { Value } \\
\text { (FIC/in) }\end{array}$ & Comments & $\begin{array}{l}\text { Box } \\
\text { Number }\end{array}$ \\
\hline $105 S x$ & $4 / 27 / 76$ & & & & & 252 & FIC QA'd B 252.4 A 251.85 & 61236 \\
\hline $105 \mathrm{SX}$ & $4 / 28 / 76$ & & & 7.3 & & 259.3 & Received slurry & 61236 \\
\hline $105 S X$ & $4 / 29 / 76$ & & & 14.6 & & 273.9 & Received slurry & 61236 \\
\hline 105SX & $5 / 5 / 76$ & & & & & 274.5 & & 61236 \\
\hline $1055 x$ & $5 / 18 / 76$ & & & & & 275.3 & $\begin{array}{l}\text { Tank last active on } 4 / 30 / 76 \text { as a slurry } \\
\text { receiver read a } 273.5 \text { to } 274 \text { after } \\
\text { receiving slurry. Readings have been } \\
\text { slowly increasing since and have been } \\
\text { slightly erratic. }\end{array}$ & 61236 \\
\hline $105 \mathrm{SX}$ & $6 / 2 / 76$ & & & & & 275.8 & & 61236 \\
\hline $105 \mathrm{SX}$ & $6 / 9 / 76$ & & & & & 276 & Flushed B 276.1 A 276.4 & 61236 \\
\hline $105 S x$ & $6 / 15 / 76$ & & & & & 276.4 & $\begin{array}{l}\text { Last active as slurry receiver on } 4 / 30 / 76 \\
\text { read between } 273.5 \text { to } 274 \text { afterwards, } \\
\text { is slightly erratic and has been } \\
\text { increasing ever since. }\end{array}$ & 61236 \\
\hline $1055 \mathrm{X}$ & $6 / 28 / 76$ & & & & & 276.9 & Flushed B 276.85 A 276.9 & 61236 \\
\hline $105 S x$ & $6 / 30 / 76$ & & & & & 276.75 & & 61236 \\
\hline $105 S x$ & $7 / 5 / 76$ & & & & & 276.8 & Flushed B 276.4 A 276.8 & 61236 \\
\hline $105 S x$ & $7 / 6 / 76$ & & & & & 276.6 & Flushed B 276.35 A 276.85 & 61236 \\
\hline $105 S X$ & $7 / 19 / 76$ & & & & & 276.8 & Flushed B 277 A 276.8 & 61236 \\
\hline $1055 x$ & $7 / 28 / 76$ & & & & & 277.3 & & 61236 \\
\hline $1055 \mathrm{X}$ & $8 / 2 / 76$ & & & & & 276.5 & FIC repaired (New Motor) & 61236 \\
\hline $105 s x$ & $8 / 3 / 76$ & & & & & 276.5 & $\begin{array}{l}\text { Manual tape feels like it is hitting } \\
\text { saltcake-can get continuity from } 23^{\prime}- \\
1 / 4^{\prime \prime} \text { to } 22^{\prime}-111 / 2^{\prime \prime} \text {, depending on where } \\
\text { the tape lands }\end{array}$ & 61236 \\
\hline $105 S X$ & $8 / 12 / 76$ & & & 6.55 & & 283.3 & Active & 61236 \\
\hline $1058 \mathrm{X}$ & $8 / 13 / 76$ & & & & & 283.1 & Received slurry & 612,36 \\
\hline $105 S X$ & $8 / 14 / 76$ & & & & & 283.25 & Flushed B 283.9 A 283.25 & 61236 \\
\hline $105 S x$ & $8 / 20 / 76$ & & & & & 283 & $\begin{array}{l}\text { Poured a little water down tape to get } \\
\text { reading, feels as if there may be sludge. }\end{array}$ & 61236 \\
\hline $105 S X$ & $9 / 1 / 76$ & & & & & 283.6 & & 61236 \\
\hline $105 S x$ & $10 / 1 / 76$ & & & & & 285 & & 61236 \\
\hline $105 S X$ & $10 / 12 / 76$ & & & & & 284.7 & Flushed and QA'd B 285.3A 284.7 & 61236 \\
\hline $105 S X$ & $11 / 1 / 76$ & & & & & 285.4 & & 61236 \\
\hline $105 S x$ & $11 / 4 / 76$ & & & & & 285.6 & $\begin{array}{l}\text { Pressure tested from 104SX to SXB, } \\
\text { SXB drains to } 102 S X \text {. Also opened up } \\
105 S X \text { in prep for test. }\end{array}$ & 61236 \\
\hline $1055 X$ & $11 / 8 / 76$ & & 1025 & & 2.6 & 283 & & 61236 \\
\hline $105 \mathrm{Sx}$ & $11 / 11 / 76$ & & & & & 285.4 & $\begin{array}{l}242 S \text { Slurry line to } 105 S X \text { leak checked, } \\
\text { FIC is erratic, probably a lot of sludge } \\
\text { floating on top. }\end{array}$ & 61236 \\
\hline $105 S \mathrm{X}$ & $11 / 12 / 76$ & & & 42.25 & & 327.65 & Slurry receiver & 61236 \\
\hline $105 \mathrm{SX}$ & $11 / 13 / 76$ & & & 27.65 & & 355.3 & Slurry receiver & 61236 \\
\hline $105 \mathrm{SX}$ & $11 / 15 / 76$ & & & & & 355.3 & Flushed B 355.3 A 355.15 & 61236 \\
\hline $105 \mathrm{SX}$ & $12 / 1 / 76$ & & & & & 357 & & 61236 \\
\hline $105 S X$ & $12 / 8 / 76$ & & & & & 357.9 & Flushed B 357.9 A 357.9 & 61236 \\
\hline $105 \mathrm{SX}$ & $12 / 28 / 76$ & & & & & 359.1 & Flushed B 359.35 A 359.1 & 61236 \\
\hline $105 \mathrm{SX}$ & $12 / 31 / 76$ & & & & & 359.2 & & 61236 \\
\hline & & & & & & & & \\
\hline SX105 & $1 / 1 / 77$ & & & & & 359.2 & & 61233 \\
\hline$S \times 105$ & $1 / 5 / 77$ & & & & & 359.7 & Photographs & \\
\hline$s \times 105$ & $1 / 6 / 77$ & & & & & 359.8 & $\begin{array}{l}\text { Flushed manual tape on } 105-\mathrm{SX} \\
\text { Before: } 30^{\prime}-1 / 4^{\prime \prime} \text {. After: } 30^{\prime}-1 / 2^{\prime \prime} \text {. }\end{array}$ & \\
\hline
\end{tabular}


HNF-SD-WM-ER-693, Rev. 0

241-SX-105 Waste Level History 1973 to 1980

\begin{tabular}{|c|c|c|c|c|c|c|c|c|}
\hline $\begin{array}{c}\text { Tank } \\
\text { Number }\end{array}$ & Date & $\begin{array}{l}\text { Transfer } \\
\text { Source }\end{array}$ & $\begin{array}{c}\text { Transfer } \\
\text { Destination }\end{array}$ & $\begin{array}{l}\text { Tank } \\
\text { Addition } \\
\text { Value } \\
\text { (inches) }\end{array}$ & $\begin{array}{c}\text { Tank } \\
\text { Reduction } \\
\text { Value } \\
\text { (inches) }\end{array}$ & $\begin{array}{l}\text { Waste } \\
\text { Level } \\
\text { Value } \\
\text { (FIC/in) } \\
\end{array}$ & Comments & $\begin{array}{l}\text { Box } \\
\text { Number }\end{array}$ \\
\hline SX105 & $1 / 19 / 77$ & & & & & 361.2 & & \\
\hline $\mathrm{S} \times 105$ & $1 / 21 / 77$ & & $\$ 102$ & & 10.5 & 350.6 & & 61233 \\
\hline $\mathbf{S \times 1 0 5}$ & $1 / 25 / 77$ & & & & & 350.6 & Baseline. & \\
\hline SX105 & $2 / 1 / 77$ & . & & & & 351.2 & & 61233 \\
\hline SX105 & $2 / 10 / 77$ & & & & & 352.5 & FIC flushed, B: $352.1, A: 352.5$. & 61233 \\
\hline SX105 & $2 / 15 / 77$ & & & & & 352.5 & & 61233 \\
\hline SX105 & $2 / 16 / 77$ & S101 & & 6.2 & & 358.7 & & 61233 \\
\hline SX105 & $2 / 17 / 77$ & & & & & 358.7 & Baseline. & \\
\hline SX105 & $3 / 1 / 77$ & & & & & 358.9 & & 61233 \\
\hline SX105 & $4 / 1 / 77$ & & & & & 361 & & 61233 \\
\hline SX105 & $4 / 6 / 77$ & & & & & 361.2 & FIC flushed, $\mathrm{B}: 360.6, \mathrm{~A}: 361.2$. & 61233 \\
\hline SX105 & $4 / 8 / 77$ & & & & & 361.1 & & \\
\hline SX105 & $4 / 9 / 77$ & & SY102 & & 17.7 & 343.4 & & 61233 \\
\hline $5 \times 105$ & $4 / 10 / 77$ & & SY102 & & 24.3 & 319.1 & & 61233 \\
\hline SX105 & $4 / 11 / 77$ & & SY102 & & 40.3 & 278.8 & & 61233 \\
\hline$S \times 105$ & $4 / 12 / 77$ & & SY102 & & 12.3 & 266.5 & & 61233 \\
\hline SX105 & $4 / 13 / 77$ & & & & & 266.4 & Baseline & \\
\hline SX105 & $5 / 1 / 77$ & & & & & 267.05 & & 61233 \\
\hline$S \times 105$ & $5 / 17 / 77$ & & & & & 265.9 & $\begin{array}{l}12-8 \text { shift entry. Manual reading; is not } \\
\text { printing on computer. Baseline is } 266.4 \\
\text { date } 4-28-77, \text { the allowable decrease is } \\
.5 " \text {. The current reading is } 5^{\prime \prime} \text { below the } \\
\text { baseline, will watch it the rest of the } \\
\text { shift. }\end{array}$ & 61233 \\
\hline$s \times 105$ & $5 / 17 / 77$ & & & & & 266.6 & $\begin{array}{l}\text { FIC QA'd, B: } 267.00, A: 266.60 . \text { New } \\
\text { counter installed. }\end{array}$ & 61233 \\
\hline $\mathrm{s} \times 105$ & $5 / 19 / 77$ & & & & & 266.5 & $\begin{array}{l}12-8 \text { shift entry. } 105 S X \text { has been a little } \\
\text { erratic since it was QA'd on 5-17-77. } \\
\text { Baseline is } 266.4 \text { dated } 4-28-77 \text {. }\end{array}$ & 61233 \\
\hline $\mathrm{S} \times 105$ & $5 / 23 / 77$ & & & & & 265.8 & $\begin{array}{l}\text { 105-SX has decreased some, so will get } \\
\text { pictures in AM. Decrease has exceeded } \\
.5 " \text { criteria. Ocurrence Report } 77-44 .\end{array}$ & 61233 \\
\hline$S \times 105$ & $5 / 24 / 77$ & & & & & 265.4 & $\begin{array}{l}\text { Picture taken in 105-SX this PM. } \\
\text { Heavily fissured crust. }\end{array}$ & 61233 \\
\hline$S \times 105$ & $5 / 25 / 77$ & & & & & & & \\
\hline SX105 & $5 / 27 / 77$ & & . & & & 266 & $\begin{array}{l}\text { Gave a deep FIC flush with hot water: } \\
\text { B: } 265.9, \text { A: } 266.0 \text {. }\end{array}$ & 61233 \\
\hline$S \times 105$ & $5 / 28 / 77$ & & & & & 265.4 & $\begin{array}{l}105-\mathrm{SX} \text { FIC was given a good hot } \\
\text { water flush on } 5-27 \ldots ., \text { but that may be } \\
\text { the reason for the current drop. }\end{array}$ & 61233 \\
\hline $5 \times 105$ & $5 / 29 / 77$ & slurry & & 10.6 & & 276 & & 61233 \\
\hline SX105 & $5 / 30 / 77$ & slurry & & 20.1 & & 296.1 & & 61233 \\
\hline SX105 & $5 / 31 / 77$ & slurry & & 9.35 & & 305.45 & & 61233 \\
\hline SX105 & $6 / 2 / 77$ & slurry & & 5.85 & & 311.3 & & 61233 \\
\hline SX105 & $6 / 3 / 77$ & slutry & & 25.6 & & 336.9 & & 61233 \\
\hline SX105 & $6 / 4 / 77$ & slurty & & 18.1 & & 355 & & 61233 \\
\hline SX105 & $6 / 5 / 77$ & slurry & & & & & & \\
\hline SX105 & $6 / 6 / 77$ & & SY102 & & 30.5 & 324.5 & & 61233 \\
\hline SX105 & $6 / 7 / 77$ & & SY102 & & 47.4 & 277.1 & & 61233 \\
\hline SX105 & $6 / 8 / 77$ & slurry & & 29 & & 306.1 & & 61233 \\
\hline SX105 & $6 / 9 / 77$ & slurry & & 36.2 & & 342.3 & & 61233 \\
\hline SX105 & $6 / 10 / 77$ & & S107 & & 1.5 & 340.8 & & 61233 \\
\hline $\mathrm{S} \times 105$ & $6 / 11 / 77$ & & $\$ 107$ & & 17.4 & 323.4 & & 61233 \\
\hline SX105 & $6 / 12 / 77$ & slurry & & 15.4 & & 338.8 & & 61233 \\
\hline
\end{tabular}


241-SX-105 Waste Level History

HNF-SD-WM-ER-693, Rev. 0

1973 to 1980

\begin{tabular}{|c|c|c|c|c|c|c|c|c|}
\hline $\begin{array}{c}\text { Tank } \\
\text { Number }\end{array}$ & Date & $\begin{array}{l}\text { Transfer } \\
\text { Source }\end{array}$ & $\begin{array}{c}\text { Transfer } \\
\text { Destination }\end{array}$ & $\begin{array}{c}\text { Tank } \\
\text { Addition } \\
\text { Value } \\
\text { (inches) }\end{array}$ & $\begin{array}{c}\text { Tank } \\
\text { Reduction } \\
\text { Value } \\
\text { (inches) }\end{array}$ & $\begin{array}{l}\text { Waste } \\
\text { Level } \\
\text { Value } \\
\text { (FIC/in) } \\
\end{array}$ & Comments & $\begin{array}{l}\text { Box } \\
\text { Number }\end{array}$ \\
\hline SX105 & $6 / 13 / 77$ & slurry & & 10.9 & & 349.7 & & 61233 \\
\hline $5 \times 105$ & $6 / 14 / 77$ & & S107 & & 9.9 & 339.8 & & 61233 \\
\hline SX105 & $6 / 15 / 77$ & slurry & & 6.5 & & 346.3 & & 61233 \\
\hline SX105 & $6 / 29 / 77$ & & & & & 347.7 & FIC flushed, $B: 348.1, A ; 347.7$. & 61233 \\
\hline SX105 & $7 / 1 / 77$ & & & & & 348 & & 61233 \\
\hline SX105 & $7 / 5 / 77$ & & & & & 348.3 & & \\
\hline SX105 & $7 / 7 / 77$ & & & & & 348.5 & Baseline & \\
\hline SX105 & $7 / 8 / 77$ & & & & & 348.8 & & 61233 \\
\hline SX105 & $7 / 25 / 77$ & & & & & 351.1 & FIC flushed, $B: 351.25, A: 351.1$. & 61233 \\
\hline sx105 & $7 / 27 / 77$ & & & & & 350.9 & $\begin{array}{l}\text { Rechecked 105SXFIC - This has } \\
\text { shown quite a bit of change the last two } \\
\text { weeks. }\end{array}$ & 61233 \\
\hline SX105 & $8 / 1 / 77$ & & & & & 351.7 & & 61233 \\
\hline SX105 & $8 / 3 / 77$ & & & & & 351.7 & $\begin{array}{l}\text { FIC exceeds } 3^{\prime \prime} \text { increase criteria. } \\
\text { Occurrence Report } 77-133 \text {. }\end{array}$ & \\
\hline SX105 & $8 / 4 / 77$ & & & & & 352.8 & FIC flushed, $B: 353.1, A: 352.8$. & 61233 \\
\hline SX105 & $8 / 13 / 77$ & & & & & 353.75 & $\begin{array}{l}\text { Increase. Crane crew pressure } \\
\text { checking lines into 105-SX.. }\end{array}$ & 61233 \\
\hline $5 \times 105$ & $8 / 20 / 77$ & & & & & 353.6 & & \\
\hline SX105 & $8 / 20 / 77$ & & $\$ 107$ & & 21.8 & 331.8 & 12-8 shift reading & 61233 \\
\hline SX105 & $8 / 21 / 77$ & & S107 & & & 331.8 & & 61233 \\
\hline SX105 & $8 / 23 / 77$ & & & & & 331.1 & $\begin{array}{l}\text { Decrease. } 105-\mathrm{SX} \text { double checked. It } \\
\text { must have floated off a crust or icicle } \\
\text { broke off. }\end{array}$ & 61233 \\
\hline SX105 & $8 / 23 / 77$ & & & & & 330.8 & FIC flushed, $B: 331.1, A: 330.8$. & 61233 \\
\hline SX105 & $8 / 24 / 77$ & & S107 & & 23 & 299.5 & & 61233 \\
\hline SX105 & $8 / 25 / 77$ & & 5107 & & & & & \\
\hline SX105 & $8 / 28 / 77$ & slurry & & 29.8 & & 328.6 & & 61233 \\
\hline SX105 & $8 / 29 / 77$ & & & 8.7 & & 343.7 & & 61233 \\
\hline SX105 & $8 / 29 / 77$ & slurry & & & 23.8 & 352.4 & & 61233 \\
\hline SX105 & $8 / 30 / 77$ & & $\$ 101$ & & 7.2 & 336.5 & Baseline. & 61233 \\
\hline sx105 & $9 / 1 / 77$ & & & & & 337.1 & $\begin{array}{l}\text { Water added to } 105-S X \text { while repairing } \\
\text { valves. }\end{array}$ & 61233 \\
\hline SX105 & $9 / 2 / 77$ & & S101 & & 27.6 & 309.5 & & 61233 \\
\hline SX105 & $9 / 4 / 77$ & & & & & 309.7 & $\begin{array}{l}\text { FIC needs flush, tank has salt cake. } \\
\text { Baseline. }\end{array}$ & 61233 \\
\hline SX105 & $9 / 6 / 77$ & slurry & & 7.4 & & 317.2 & & 61233 \\
\hline SX105 & $9 / 7 \pi 7$ & slurry & & 7.7 & & 324.9 & & 61233 \\
\hline $5 \times 105$ & $9 / 8 / 77$ & slurry & & 13.5 & & 338.4 & & 61233 \\
\hline$S \times 105$ & $9 / 9 / 77$ & slurry & & 7.1 & & 345.5 & & 61233 \\
\hline $5 \times 105$ & $9 / 10 / 77$ & sluriy & & 8.5 & & 354 & & 61233 \\
\hline SX105 & $9 / 11 / 77$ & & $\$ 101$ & & 31.6 & 322.4 & & 61233 \\
\hline sX105 & 9/17/77 & slurry & & 4.7 & & 327.4 & & 61233 \\
\hline SX105 & $9 / 18 / 77$ & sluriy & & 3.9 & & 331.3 & & 61233 \\
\hline SX105 & $9 / 19 / 77$ & slurny & & 2.1 & & 333.4 & & 61233 \\
\hline$S \times 105$ & $9 / 20 / 77$ & slurry & & 3.2 & & 336.6 & & 61233 \\
\hline SX105 & $9 / 21 / 77$ & slurry & & 7.3 & & 343.9 & & 61233 \\
\hline $5 \times 105$ & $9 / 22 / 77$ & slurry & & 2.05 & & 345.95 & & 61233 \\
\hline SX105 & $9 / 23 / 77$ & slurry & & 0.85 & & 346.8 & & 61233 \\
\hline $5 \times 105$ & $9 / 24 / 77$ & slurry & & 2.1 & & 348.9 & & 61233 \\
\hline $5 \times 105$ & $9 / 25 / 77$ & slurry & & 5.6 & & 354.5 & & 61233 \\
\hline$S \times 105$ & $9 / 26 / 77$ & & & & & 354.6 & & \\
\hline$S \times 105$ & $9 / 28 / 77$ & & & & & 354.6 & Baseline & \\
\hline SX105 & $9 / 29 / 77$ & & & & & 355.35 & 105-SX was a slurty receiver. & 61233 \\
\hline SX105 & $10 / 1 / 77$ & & & & & 355.45 & & 61233 \\
\hline
\end{tabular}


241-SX-105 Waste Level History

1973 to 1980

\begin{tabular}{|c|c|c|c|c|c|c|c|c|}
\hline $\begin{array}{l}\text { Tank } \\
\text { Number }\end{array}$ & Date & $\begin{array}{l}\text { Transfer } \\
\text { Source }\end{array}$ & $\begin{array}{c}\text { Transfer } \\
\text { Destination }\end{array}$ & $\begin{array}{l}\text { Tank } \\
\text { Addition } \\
\text { Value } \\
\text { (inches) }\end{array}$ & \begin{tabular}{|l} 
Tank \\
Reduction \\
Value \\
(inches)
\end{tabular} & $\begin{array}{c}\text { Waste } \\
\text { Level } \\
\text { Value } \\
\text { (FIC/in) }\end{array}$ & Comments & $\begin{array}{l}\text { Box } \\
\text { Number }\end{array}$ \\
\hline SX105 & $10 / 2 / 77$ & & & & & 355.8 & $\begin{array}{l}\text { 4-12 shift reading. Increase of .35" } \\
\text { (FIC, } .25 \text { for MT) since yesterday. } 105- \\
\text { SX raised tape manually \& still get } \\
355.75 \text {. May be some flush } \mathrm{H} 2 \mathrm{O} \text { thru } \\
\text { slurry line on } 10-1-77 \text {. }\end{array}$ & 61233 \\
\hline SX105 & $10 / 4 / 77$ & & & & & 356.95 & $\begin{array}{l}8-4 \text { shift reading. FIC double checked. } \\
\text { Rise may be due to flushes in SX-B } \\
\text { valve pit jumpers on days 10-3-77 also } \\
\text { flushed in SX-A on 10-3-77. }\end{array}$ & 61233 \\
\hline$\$ \times 105$ & $10 / 4 / 77$ & & & & & 355.85 & $\begin{array}{l}4-12 \text { shift reading. FIC flushed, } B \text { : } \\
356.0, A: 355.85 \text {. }\end{array}$ & 61233 \\
\hline SX105 & $10 / 10 / 77$ & & & & & 357.4 & & 61233 \\
\hline SX105 & $10 / 11 / 77$ & & & & & 357.2 & FIC flushed, $\mathrm{B}: 357.5, \mathrm{~A}: 357.2$. & 61233 \\
\hline$S \times 105$ & $10 / 12 / 77$ & & & & & 358.4 & $\begin{array}{l}\text { FIC is up quite a bit. May be shifting of } \\
\text { crust. Baseline is } 354.6 \text { on } 9-28-77 \text {. } \\
\text { Allowed } 3 " \text { rise. Computer read } 357.9 \text { at } \\
1500 \text { and } 358.4 \text { at } 1600 \text {, has held steady } \\
\text { since. }\end{array}$ & 61233 \\
\hline SX105 & $10 / 13 / 77$ & & & & & 357.5 & FIC flushed, $8: 358.45, A: 357.5$. & 61233 \\
\hline SX105 & $10 / 15 / 77$ & & & & & 358.8 & $\begin{array}{l}\text { FIC exceeds } 3^{\prime \prime} \text { increase criteria. See } \\
\text { O.R. } 77-184 \text {. See remarks in next two } \\
\text { cells below. }\end{array}$ & 61233 \\
\hline & & & & & & & $\begin{array}{l}\text { Rechecked (swing reading of } 359.2 \text { ), } \\
\text { came up on graveyard on } 10-15 \text { to } 358.6 \\
\text { to } 358.8 \text { today. Computer P.O. showed } \\
\text { grad. increase to } 359.7 @ 1800 \text { on } 10- \\
15 \text { and dropped back down } 359.2 @ \\
1900,359.1 @ 2000 \text { and } 2100 \text {. Water is } \\
\text { off }\end{array}$ & . \\
\hline & & & . & & & & $\begin{array}{l}\text { in farm, no explanation for rise. Manual } \\
\text { tape is up } 1 " \text {, can feel very definite crust. } \\
\text { Crust might be moving. Was flushed on } \\
10-13 \text {, before } 358.45 \text {, after } 357.5 \text {. } \\
\text { Baseline is } 354.6 \mathrm{dtd} 9-28-77 \text {. Flushed } \\
\text { it per TFS } 9 \text { SW } 10-15 \text { ) .Before } 359.5 \\
\text { After } 359.2 \text {. }\end{array}$ & \\
\hline SX105 & $10 / 17 / 77$ & 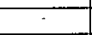 & & & & 357.9 & FIC flushed, B: $359.9, A: 357.9$ & 61233 \\
\hline$s \times 105$ & $10 / 19 / 77$ & & & & & 358.2 & $\begin{array}{l}\text { Photographs taken as noted in O.R. } 77- \\
184 \text {. }\end{array}$ & 61233 \\
\hline sX105 & $11 / 1 / 77$ & & & & & 360.3 & $\begin{array}{l}\text { Increase. Pressure testing to } 105-\mathrm{SX} \& \mathrm{a} \\
104-\mathrm{SX} \text {. }\end{array}$ & 61233 \\
\hline SX105 & $11 / 2 / 77$ & & & & & 360.3 & Baseline & \\
\hline$S \times 105$ & $11 / 11 / 77$ & & & & & 361.7 & FIC flushed, $\mathrm{B}: 362.0, \mathrm{~A}: 361.7$. & 61233 \\
\hline $\mathrm{S} \times 105$ & $11 / 12 / 77$ & & & & & 361.05 & $\begin{array}{l}\text { 12-8 shift entry. Rechecked - reading } \\
\text { dropped to } 361.0 \text { on } 2200 \text { printout - base } \\
360.3 \text {. Previous reading was } 361.8 \text {. }\end{array}$ & 61233 \\
\hline SX105 & $11 / 13 / 77$ & & & & & 361.9 & $\begin{array}{l}\text { 105-SX has alarmed at } 360.0 \text { twice over } \\
\text { night - surveillance is aware. }\end{array}$ & 61233 \\
\hline$S \times 105$ & $11 / 14 / 77$ & & & & & 362 & $\begin{array}{l}\text { New limit on } 105-S \times \text { has been set to } \\
365^{\prime \prime} \text { until Dec. } 1 \text {. Also systems people } \\
\text { are in the process of changing the LL } \\
\text { alarm point. }\end{array}$ & 61233 \\
\hline SX105 & $11 / 27 / 77$ & & & & & 362.4 & & 61233 \\
\hline
\end{tabular}


241-SX-105 Waste Level History

1973 to 1980

\begin{tabular}{|c|c|c|c|c|c|c|c|c|}
\hline $\begin{array}{l}\text { Tank } \\
\text { Number }\end{array}$ & Date & $\begin{array}{l}\text { Transfer } \\
\text { Source } \\
\end{array}$ & $\begin{array}{c}\text { Transfer } \\
\text { Destination }\end{array}$ & $\begin{array}{c}\text { Tank } \\
\text { Addition } \\
\text { Value } \\
\text { (inches) } \\
\end{array}$ & $\begin{array}{c}\text { Tank } \\
\text { Reduction } \\
\text { Value } \\
\text { (inches) }\end{array}$ & $\begin{array}{l}\text { Waste } \\
\text { Level } \\
\text { Value } \\
\text { (FIC/in) }\end{array}$ & Comments & $\begin{array}{l}\text { Box } \\
\text { Number }\end{array}$ \\
\hline sX105 & $11 / 28 / 77$ & & & & & 364.3 & $\begin{array}{l}\text { When first read, it was } 362.95 \text {. } \\
\text { Operator raised and lowered it a couple } \\
\text { of times, now it has leveled out at } 364.3 \text {. } \\
\text { The manual tape is still the same. At } \\
2100 \text { print out on } 11-28 \text { it read } 362.2 \text {, no } \\
\text { reading at } 2200 \text {, then } 2300362.9 \text { and } \\
\text { stayed there }\end{array}$ & 61233 \\
\hline SX105 & $11 / 29 / 77$ & & & - & & 362.35 & $\begin{array}{l}\text { Flushed, B: } 364.45, A: 362.35,105-S X \\
\text { base is } 360.3 \text {. }\end{array}$ & 61233 \\
\hline SX105 & $12 / 1 / 77$ & & & & & 362.25 & & 61233 \\
\hline$\$ \times 105$ & $12 / 1 / 77$ & & SY102 & & 1.7 & 360.55 & 12-8 shift reading. & 61233 \\
\hline SX105 & $12 / 2 / 77$ & & SY102 & & 17.55 & 343 & & 61233 \\
\hline SX105 & $12 / 3 / 77$ & & SY102 & & 35.5 & 307.5 & 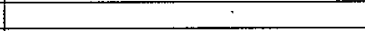 & 61233 \\
\hline SX105 & $12 / 4 / 77$ & & SY102 & & 27.5 & 280 & & 61233 \\
\hline SX105 & $12 / 5 / 77$ & & SY102 & & 5.6 & 274.4 & Graveyard entry (12-8 shift) & 61233 \\
\hline SX105 & $12 / 6 / 77$ & & & & & 275.5 & $\begin{array}{l}\text { Day shift reading (8-4). Mud sliding in. } \\
\text { (Transfer to } 102 S Y \text { is done but don't } \\
\text { understand "mud sliding in" comment.) }\end{array}$ & 61233 \\
\hline SX105 & $12 / 8 / 77$ & & & & & 275.6 & Baseline. & \\
\hline SX105 & $12 / 11 / 77$ & & & & & 276.4 & FIC has been erratic. & 61233 \\
\hline$S \times 105$ & $12 / 13 / 77$ & & & & & 276.85 & $\begin{array}{l}\text { It rained very hard, all tanks up a little. } \\
\text { Day shift worked in SX-A pit today. } \\
\text { Could account for some of the LL } \\
\text { increases. }\end{array}$ & 61233 \\
\hline SX105 & $12 / 31 / 77$ & & & & : & 277.3 & & 61233 \\
\hline & 20 & & & & & 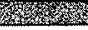 & 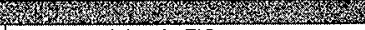 & \\
\hline SX105 & $1 / 1 / 78$ & & & & & 277.30 & Monthly tank level - FIC & 68654 \\
\hline $5 \times 105$ & $2 / 1 / 78$ & & & & & 278.00 & Monthly tank level - FIC & 68654 \\
\hline SX105 & $3 / 1 / 78$ & & & & & 277.25 & Monthly tank level - FIC & 68654 \\
\hline SX105 & $4 / 1 / 78$ & & & & & 277.10 & Monthly tank level - FIC & 68654 \\
\hline SX105 & $4 / 13 / 78$ & & & & & 276.30 & Flushed FIC - FIC & 68654 \\
\hline $5 \times 105$ & $5 / 1 / 78$ & & & & & 276.75 & Monthly tank level - FIC & 68654 \\
\hline $\mathrm{S} \times 105$ & $6 / 1 / 78$ & & & & & 277.30 & Monthly tank level - FIC & 68654 \\
\hline$S \times 105$ & $6 / 19 / 78$ & & & & & 277.45 & QA'd FIC (MT unchanged) & 68654 \\
\hline$S \times 105$ & $7 / 1 / 78$ & & & & & 277.00 & Monthly tank level - FIC & 68654 \\
\hline$S \times 105$ & $8 / 1 / 78$ & & & & & 277.00 & Monthly tank level - FIC & 68654 \\
\hline SX105 & $8 / 21 / 78$ & & & & & 277.35 & & 68654 \\
\hline $\mathrm{S} \times 105$ & $8 / 22 / 78$ & & & & & 277.50 & & 68654 \\
\hline$S \times 105$ & $9 / 1 / 78$ & & & & & 277.60 & Monthly tank level - FIC & 68654 \\
\hline SX105 & $9 / 20 / 78$ & & & & & 277.50 & Photogarphs. & 68654 \\
\hline SX105 & $9 / 22 / 78$ & & & & & 275.55 & Flushed FIC (MT unchanged) & 68654 \\
\hline SX105 & $9 / 23 / 78$ & & & & & 275.10 & Flushed FIC (MT unchanged) & 68654 \\
\hline$S \times 105$ & $10 / 1 / 78$ & & & & & 275.65 & Monthly tank level - FIC & 68654 \\
\hline$S \times 105$ & $11 / 1 / 78$ & & & & & 275.50 & Monthly tank level - FIC & 68654 \\
\hline$S \times 105$ & $12 / 1 / 78$ & & & & & 276.30 & Monthly tank level - FIC & 68654 \\
\hline SX105 & $12 / 5 / 78$ & & & & & 275.10 & Flushed FIC & 68654 \\
\hline SX105 & $12 / 7 / 78$ & & & & & 276.80 & FIC flushed and calibrated & 68654 \\
\hline SX105 & $12 / 31 / 78$ & & & & & 276.10 & Year end tank level - FIC & 68654 \\
\hline & & & & & & & 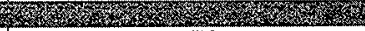 & \\
\hline SX105 & $1 / 1 / 79$ & & & & & 276.10 & Monthly tank level - FIC & 74293 \\
\hline SX105 & $1 / 11 / 79$ & & & & & 276.75 & Unknown rise - FIC (MT unchanged) & 74293 \\
\hline SX105 & $1 / 12 / 79$ & & & & & 276.40 & Unknown drop - FIC (MT unchanged) & 74293 \\
\hline SX105 & $1 / 22 / 79$ & & & & & 276.65 & Unknown drop - FIC (MT unchanged) & 74293 \\
\hline SX105 & $2 / 1 / 79$ & & & & & 276.90 & Monthly tank level - FIC & 74293 \\
\hline
\end{tabular}


241-SX-105 Waste Level History

HNF-SD-WM-ER-693, Rev. 0 1973 to 1980

\begin{tabular}{|c|c|c|c|c|c|c|c|c|}
\hline $\begin{array}{l}\text { Tank } \\
\text { Number }\end{array}$ & Date & $\begin{array}{l}\text { Transfer } \\
\text { Source }\end{array}$ & $\begin{array}{c}\text { Transfer } \\
\text { Destination }\end{array}$ & $\begin{array}{l}\text { Tank } \\
\text { Addition } \\
\text { Value } \\
\text { (inches) }\end{array}$ & $\begin{array}{l}\text { Tank } \\
\text { Reduction } \\
\text { Value } \\
\text { (inches) }\end{array}$ & $\begin{array}{l}\text { Waste } \\
\text { Level } \\
\text { Value } \\
\text { (FIC/in) }\end{array}$ & Comments & $\begin{array}{l}\text { Box } \\
\text { Number }\end{array}$ \\
\hline SX105 & $3 / 1 / 79$ & & & & & 276.80 & Monthly tank level - FIC & 74293 \\
\hline SX105 & $4 / 1 / 79$ & & & & & 276.70 & Monthly tank level - FIC & 74293 \\
\hline $\mathrm{S} \times 105$ & $5 / 1 / 79$ & & & & & 277.65 & Monthly tank level - FIC & 74293 \\
\hline$S \times 105$ & $6 / 1 / 79$ & & & & & 277.80 & Monthly tank level - FIC & 74293 \\
\hline$S \times 105$ & $6 / 19 / 79$ & & & & & 278.10 & Rise in FIC (flushed) & 74293 \\
\hline SX105 & $7 / 1 / 79$ & & & & & 278.50 & Monthly tank level - FIC & 74293 \\
\hline $5 \times 105$ & $8 / 1 / 79$ & & & & & 275.90 & Monthly tank level - FIC & 74293 \\
\hline SX105 & $8 / 29 / 79$ & & & & & 278.70 & Rise, FIC flushed - FIC & 74293 \\
\hline SX105 & $9 / 1 / 79$ & & & & & 278.15 & Monthly tank level - FIC & 74293 \\
\hline $5 \times 105$ & $10 / 1 / 79$ & & & & & 278.30 & Monthly tank level - FIC & 74293 \\
\hline SX105 & $10 / 12 / 79$ & & & & & 278.95 & Drop, FIC flushed - FIC & 74293 \\
\hline SX105 & $10 / 18 / 79$ & & & & & 278.55 & & 74293 \\
\hline$\$ \times 105$ & $10 / 19 / 79$ & S107 & & 24.00 & & 302.55 & Rec'd transfer - FIC & 74293 \\
\hline SX105 & $10 / 23 / 79$ & & & & & 301.60 & Rise, FIC flushed - FIC & 74293 \\
\hline SX105 & $10 / 26 / 79$ & & & & & 300.40 & Photographs & \\
\hline SX105 & $11 / 1 / 79$ & & & & & 300.10 & Monthly tank level - FIC & 74293 \\
\hline SX105 & $11 / 6 / 79$ & & & & & 299.50 & Baseline & \\
\hline$S \times 105$ & $12 / 1 / 79$ & & & & & 300.30 & Monthly tank level - FIC & 74293 \\
\hline SX105 & $12 / 17 / 79$ & & & & & 297.20 & Drop (erratic) - FIC. Photographs. & 74293 \\
\hline SX105 & $12 / 20 / 79$ & & & & & 296.90 & Photographs. & \\
\hline$S \times 105$ & $12 / 31 / 79$ & & & & & 297.65 & Year end tank level - FIC & 74293 \\
\hline & Prom & 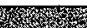 & & & & & Vis & 2 \\
\hline SX105 & $1 / 1 / 80$ & & & & & 297.8 & & 74292 \\
\hline SX105 & $1 / 10 / 80$ & & & & & 299 & $\begin{array}{l}\text { 105SX flushed before } 298.6 \text { after } \\
299.55 \text {. }\end{array}$ & 74292 \\
\hline SX105 & $1 / 17 / 80$ & & & & & 298 & Baseline & 111509 \\
\hline SX105 & $1 / 17 / 80$ & & & & & 298.7 & Baseline established at 298.0 this day. & 74292 \\
\hline SX105 & $1 / 22 / 80$ & & & & & 298.9 & & \\
\hline $5 \times 105$ & $1 / 23 / 80$ & & & & & 299 & & \\
\hline SX105 & $1 / 24 / 80$ & & & & & 299.5 & $\begin{array}{l}\text { 104-SX pumped to } 102-S Y .8-4 \text { A.M. } \\
\text { pump flushed to } 102-S Y \text { used } 1,450 \text { gal. } \\
\text { Some flush water reached } 105-S X \text {. }\end{array}$ & \\
\hline$s \times 105$ & $1 / 25 / 80$ & & & & & 299.6 & $\begin{array}{l}\text { Some flush water reported to have } \\
\text { reached } 105-\mathrm{SX} \text {. }\end{array}$ & \\
\hline$s \times 105$ & $1 / 28 / 80$ & & & & & 300 & $\mathrm{H}_{2} \mathrm{O}$ added to flush pump. & 74292 \\
\hline SX105 & $2 / 1 / 80$ & & SY102 & & 25.9 & 274.1 & $\begin{array}{l}\text { foot-inch value not given. Remarks } \\
\text { state "broken tape". }\end{array}$ & 74292 \\
\hline SX105 & $2 / 2 / 80$ & & & & & 274.5 & flush water & 74292 \\
\hline sX105 & $2 / 7 / 80$ & S103 & & & & 274.15 & 105-SX starts receiving from $103-\mathrm{S}$. & 74292 \\
\hline SX105 & $2 / 8 / 80$ & S103 & & 20.45 & & 294.6 & 105-SX rec. from 103-S. & 74292 \\
\hline SX105 & $2 / 9 / 80$ & $\mathbf{S 1 0 3}$ & & 32 & & 326.6 & 103-S to 105-SX. Baseline.I & 74292 \\
\hline$\$ \times 105$ & $2 / 11 / 80$ & & & & & 326.7 & & 74292 \\
\hline sx105 & $2 / 13 / 80$ & & & & & 326.8 & & 74292 \\
\hline $\mathrm{s} \times 105$ & $2 / 14 / 80$ & & & & & 327.2 & Baseline established @ 326.6 this day. & 111509 \\
\hline SX105 & $3 / 1 / 80$ & & & & & 327 & & 74292 \\
\hline SX105 & $3 / 21 / 80$ & & & & & 327.2 & Prior to QA. & 74292 \\
\hline SX105 & $3 / 21 / 80$ & & & & & 327.05 & After QA. & 74292 \\
\hline $\mathrm{s} \times 105$ & $4 / 1 / 80$ & & & & & 327.3 & & 74292 \\
\hline $5 \times 105$ & $4 / 27 / 80$ & & & & & 327.5 & & \\
\hline SX105 & $4 / 29 / 80$ & & & & & 327.9 & $\begin{array}{l}\text { Backflushing } 107-\mathrm{S} \text { to } 104-\mathrm{SX} \& 105- \\
\mathrm{SX} \text {; pit drains to } 102-\mathrm{SX} \text {. }\end{array}$ & 74292 \\
\hline $\mathrm{s} \times 105$ & $5 / 1 / 80$ & & & & & 327.9 & & 74292 \\
\hline$S \times 105$ & $5 / 16 / 80$ & & & & & 328.05 & Water added-pump flush. & \\
\hline
\end{tabular}


241-SX-105 Waste Level History

HNF-SD-WM-ER-693, Rev. 0

1973 to 1980

\begin{tabular}{|c|c|c|c|c|c|c|c|c|}
\hline $\begin{array}{c}\text { Tank } \\
\text { Number }\end{array}$ & Date & $\begin{array}{l}\text { Transfer } \\
\text { Source }\end{array}$ & $\begin{array}{c}\text { Transfer } \\
\text { Destination }\end{array}$ & $\begin{array}{c}\text { Tank } \\
\text { Addition } \\
\text { Value } \\
\text { (inches) }\end{array}$ & $\begin{array}{c}\text { Tank } \\
\text { Reduction } \\
\text { Value } \\
\text { (inches) }\end{array}$ & $\begin{array}{l}\text { Waste } \\
\text { Level } \\
\text { Value } \\
\text { (FIC/in) }\end{array}$ & Comments & $\begin{array}{c}\text { Box } \\
\text { Number }\end{array}$ \\
\hline SX105 & $6 / 1 / 80$ & & & & & 326.9 & & 74292 \\
\hline$\$ \times 105$ & $7 / 1 / 80$ & & & & & 326.25 & & 74292 \\
\hline $5 \times 105$ & $8 / 1 / 80$ & & & & & 326.6 & & 74292 \\
\hline$S \times 105$ & $8 / 5 / 80$ & & & & & 326.65 & $\begin{array}{l}\text { installed pump - } 1000 \text { gallons water } \\
\text { added }\end{array}$ & $\begin{array}{l}121777 \\
121861\end{array}$ \\
\hline$S \times 105$ & $8 / 7 / 80$ & & & & & 327.1 & $\begin{array}{l}\text { Lanced } 105-\mathrm{SX} \text { and installed salt well } \\
\text { screen. }\end{array}$ & 74292 \\
\hline$S \times 105$ & $8 / 26 / 80$ & & $\mathbf{S 1 0 7}$ & & 28.9 & 298.2 & & 74292 \\
\hline SX105 & $8 / 27 / 80$ & & S107 & & 37 & 261.2 & & 74292 \\
\hline$S \times 105$ & $8 / 28 / 80$ & & S107 & & 5.6 & 255.6 & & 74292 \\
\hline$\$ \times 105$ & $9 / 1 / 80$ & & & & & 255.65 & & 74292 \\
\hline$\$ \times 105$ & $9 / 2 / 80$ & & & & & 255.8 & Baseline at 255.6 & \\
\hline$\$ \times 105$ & $10 / 1 / 80$ & & & & & 255.3 & & 74292 \\
\hline$S \times 105$ & $11 / 1 / 80$ & & & . & & 256.7 & & 74292 \\
\hline SX105 & $12 / 2 / 80$ & & & & & 255.55 & & 74292 \\
\hline SX105 & $12 / 9 / 80$ & & & & & 254.5 & Baseline @ 254.5. & \\
\hline SX105 & $12 / 31 / 80$ & & & & & 254.8 & & 74292 \\
\hline
\end{tabular}


HNF-SD-WM-ER-693, Rev. 0

\section{APPENDIX F}

\section{OCCURRENCE REPORTS}

$$
\begin{aligned}
& 76-40 \\
& 76-172 \\
& 77-74 \\
& 77-133 \\
& 77-184 \\
& 79-125
\end{aligned}
$$



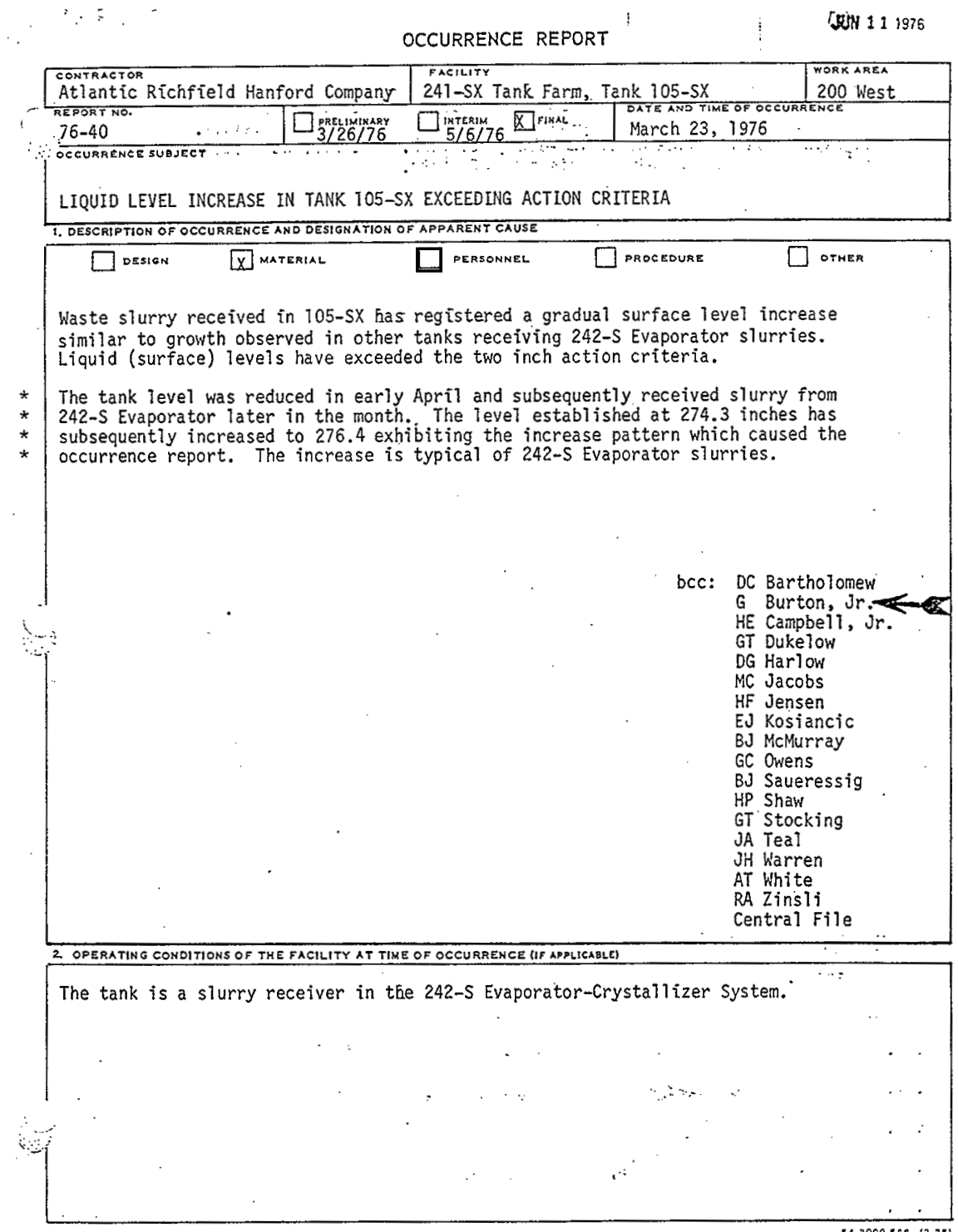
3. IMMEDIATE EVALUATION, CORRECTIVE ACTIOH TAKEN AND RESULTS

Photographs on March 10,1976 , show a smooth surface covered with solid material which is starting to. show signs of drying up with some minor cracks visible.

The photographs gave no evidence of in-leakage to tank. The surface level increase

is typical of other tanks receiving this type of Waste.

4. RECONMENDATIONS

A. TEMPOKARY CORKECTIVE ACTION

The surface level increase approached the maxtrum operating limit of 362 inches and liquid was pumped out to prevent exceeding this 1 imit.

D. PERMANENT COEKECTIVE ACTION

Continue operational controls presentiy in use for S and SX tanks containing materials exhibiting similar characteristics.

Restrict refill of tank to allow for salt formation and expansion factor. A fill

limit of 350 inches should allow sufficient room for anticipated expansion.

\begin{tabular}{|c|c|c|}
\hline $\begin{array}{c}\text { C. IS DESIGN CHANGE NECESSLFYY } \\
\square \text { YES }\end{array}$ & IF YES, WHEN & \\
\hline $\begin{array}{c}\text { D. IS FURTHUR EVALUATION NECESSLRY } \\
\square \text { YES }\end{array}$ & IF YES. EY WHOM & WHEN \\
\hline $\begin{array}{l}\text { E. PEFMANENT COARECTIVE } \\
\text { ACTION YOOE IMPLEMENTED } \\
\text { BY }\end{array}$ & & FATE \\
\hline
\end{tabular}

3. SIMILAR OCCURRENCE: EY REPORT NUMEER AND OCCUREENCE SUEJECT

*. Indicates changes in text from previous occurrence

Att.

cc: w/att.

0J Bennett, ERDA-RL.

OJ Elgert, ERDA-RL (3)

PG Rhoades, ERDA-RL

JH Straub, ERDA-RL Report issue.

$\begin{aligned} & \text { oricinared } \\ & \text { H. F. Jensen }\end{aligned}$
J. A. Tea]




\section{5-SX TANK}

\section{LIQUID LEVEL HISTORY}

\begin{tabular}{|c|c|c|c|c|}
\hline Date & $\begin{array}{l}\text { Liquid } \\
\text { Leve! } \\
\text { (inches) } \\
\end{array}$ & $\begin{array}{c}\text { Change from } \\
\text { Previous Reading } \\
\text { (inches) }\end{array}$ & $\begin{array}{c}\text { Cumulative } \\
\text { Change } \\
\text { (inches) } \\
\end{array}$ & Comments \\
\hline $\begin{array}{l}02 / 03 / 76 \\
02 / 10 / 76 \\
02 / 17 / 76\end{array}$ & $\begin{array}{l}355.40 \\
356.20 \\
357.30\end{array}$ & $\begin{array}{l}\frac{\text { Man.Tape }}{354.00} \\
354.50 \\
355.50\end{array}$ & & 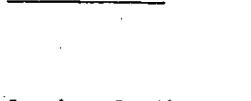 \\
\hline $02 / 24 / 76$ & 357.95 & 356.75 & & $\begin{array}{l}\text { Previous Reading } \\
\text { FIC was } 358.40 \text {, } \\
\text { FIC flushed to } \\
357.80 \text { inches. }\end{array}$ \\
\hline $\begin{array}{l}03 / 02 / 76 \\
03 / 09 / 76\end{array}$ & $\begin{array}{l}358.80 \\
359.35\end{array}$ & $\begin{array}{l}357.50 \\
358.25\end{array}$ & & \\
\hline $03 / 76 / 76$ & 356.95 & 358.75 & & $\begin{array}{l}\text { Instruments checking } \\
\text { FIC bad counter. }\end{array}$ \\
\hline $03 / 23 / 76$ & 355.00 & $\begin{array}{c}359.50 \\
?\end{array}$ & & $\begin{array}{l}\text { Instrument dept. is } \\
\text { checking the } 105-5 x \\
\text { FIC. }\end{array}$ \\
\hline $\begin{array}{l}03 / 24 / 76 \\
04 / 03 / 76\end{array}$ & $\begin{array}{l}360.85 \\
282.00\end{array}$ & 360.00 & & Transfer to $102-5$ \\
\hline $\begin{array}{l}04 / 06 / 76 \\
04 / 20 / 76 \\
04 / 24 / 76\end{array}$ & $\begin{array}{l}249.75 \\
251.20 \\
251.00\end{array}$ & $\begin{array}{l}249.25 \\
249.25 \\
249.75\end{array}$ & & Transfer to 102-s \\
\hline $\begin{array}{l}04 / 29 / 76 \\
04 / 30 / 76 \\
05 / 02 / 76\end{array}$ & $\begin{array}{l}266.10 \\
273.70 \\
274.30\end{array}$ & & & $\begin{array}{l}\text { Received Slurry } \\
\text { Received slurry }\end{array}$ \\
\hline $\begin{array}{l}05 / 15 / 76 \\
05 / 31 / 76 \\
06 / 07 / 76\end{array}$ & $\begin{array}{l}274.60 \\
275.70 \\
276.10\end{array}$ & $\begin{array}{l}274.25 \\
274.50\end{array}$ & & Baseline \\
\hline $06 / 09 / 76$ & 276.40 & & & Level after fiush \\
\hline
\end{tabular}

\footnotetext{
* Indicates changes in text from previous 0ccurrence Report issue.
} 
HNF-SD-WM-ER-693, Rev. 0

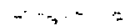

OCCURRENCE REPORT

witid 26 ;0त7

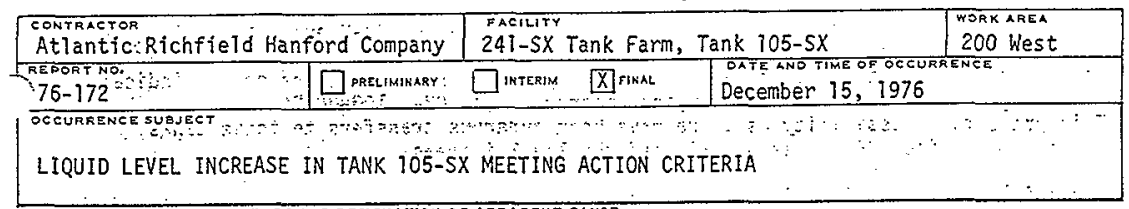

1. DESCRIPTION OF OCCURRENCE ANO DESIGNATION OF APPARENT CAUSE

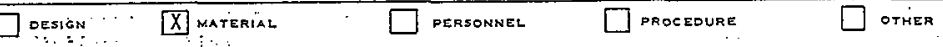

Waste slurry received in 105-SX has registered a gradual surface level increase similar to growth observed in other tanks: receiving $242-\mathrm{S}$ evaporator sturries. The increase meets the three inch action criteria. The tank received slurries from November 9, 1976 , to November 14, 1976, when the new baseline was established.

No evidence of tank in-leakage was obtained by TFO and TFPE investigations. Photographs show a considerable change in surface conditions from photographs taken in september 1976. The surface at that time presented a broken convulsed "ice-jam" view. Now, it is a smooth surface of salt - evidence of the additional slurry which has been added since September. Tank bulk temperatures are being observed and an evaluation initiated as to long term effects.

bec: DC Bartholomew
G Burton, Jr.
GT Dukelow
RA Freeman
DG Harlow
MC Jacobs
HF Jensen
EJ Kosiancic
CW Malody
S Marchetti
J Haines.

GC Owens

RD Prosser

BJ Saueressig

HP. Shaw

JA Tea?

GW Upington

JH Warren

AT White

JC Womack

RA Zinsli

Lega 1

Central File

2. OPERATING CONDITIONS OF THE FACILITY AT TIME OF OGCURRENCE (IF APPLICABLE)

The tank is a slurry recejver in the $242-5$ evaporator-crystallizer system:

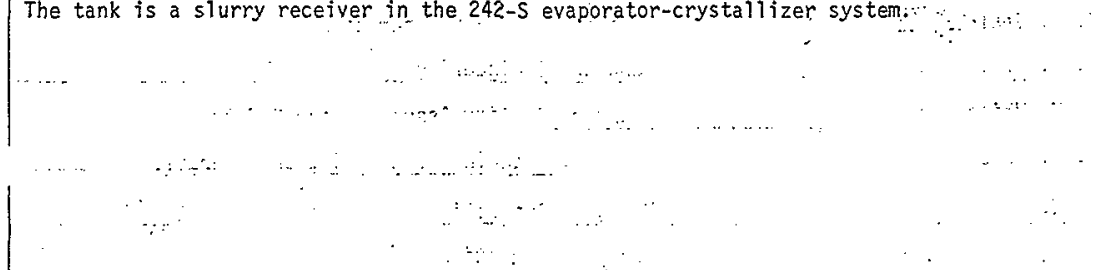

(OYer) 
3. TMMEOIATE ÉVALUATION, CORRECTIVE ACTION TAKEN AND RESULTS

The tank has experienced similar increases after receiving 242-S evaporator slurries: - An evaluation of the liquid levei plots shows similar increases, though the siope is steeper. This may indicate a change in the siurry characteristics or may indicate -in-leakage-from the transfer 1 ine. which interconnects 102-, 103-, 104, , and $105-5 x$, :-This presents a possibflity as there have been numerous transfers to these tánks during the period 105-SX has experienced the level, increase.

. Investigation of possible in-leakage has been negative. Photographs show a relatively . smooth salt surface. The increase is cons idered a typical 242-s Evaporator slurry characteristic. Similar growth has been reported repeatedly. . Tank temperatures have been screened. Increases due to temperature were evaluated and considered negitigible. L Long term effects of slow temperature increases will be studied.

$$
\text { r } x \text { is }
$$

4. RECOMMENDATIONS

A. TEMPORARY CORRECTIVE ACTION

Evaluate transfer material balances for 102-, 103-, and 104-SX over the past month. obtain other supporting evidence of tank in-leakage. No evidence of in-leakage was obtained. : The increases is considered a result of the saiting out of relativeiy low density solid particulate which floats to the surface and registers as a volume increase.

b. Perimanent conRective action

Tank is being pumped to reduce liquid level ten to eleven inches below the maximum operating limit. An evaluation of tank temperature and possible future effect on tank volumes will be initiated. This study is considered outside the scope of this occurrence.

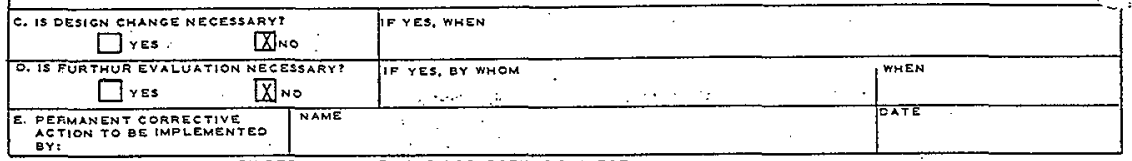

5. SIMILAR OCCURRENCE: BY REPORT NUMBER ANO OCCURRENCE SUEJECT

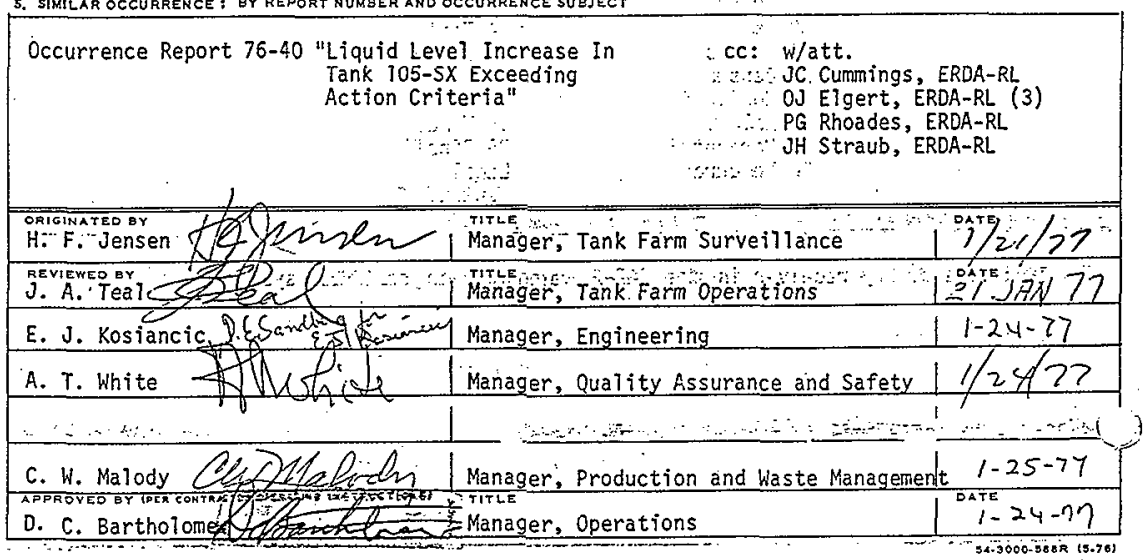


705-SX TANK

LIQUID LEVEL HISTORY

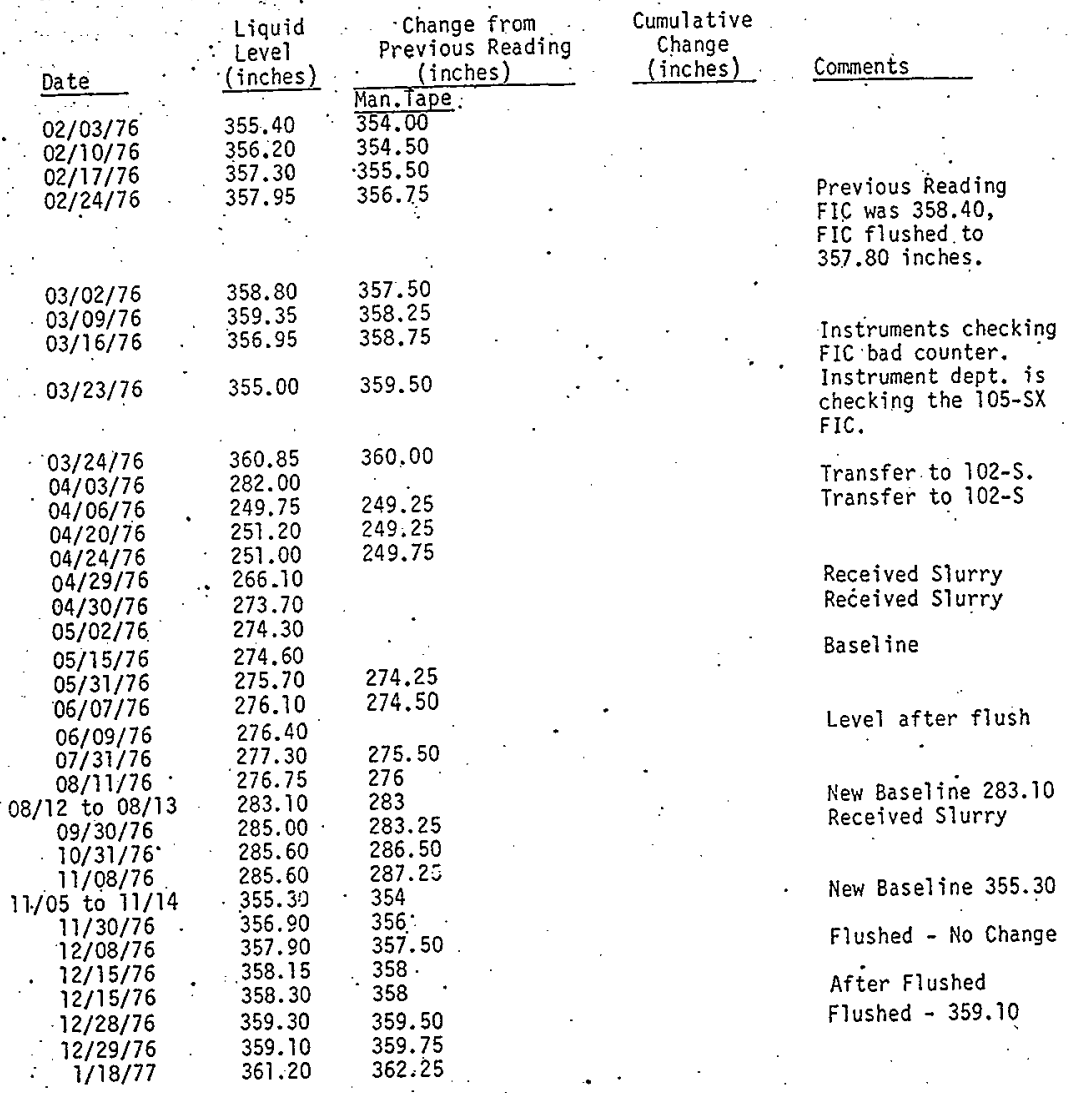

$\mathrm{HFJ}: 1 / 21 / 77$ 


\begin{tabular}{|c|c|c|c|}
\hline $\begin{array}{l}\text { CONThACTOR } \\
\text { Atlantic Richfield Hanford Company }\end{array}$ & FACILITY Tank Farm, & $\operatorname{Tank} 105-5 x$ & $\begin{array}{l}\text { WOAK AREA } \\
200 \text { HeSt }\end{array}$ \\
\hline 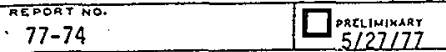 & $\square_{\text {inrekim }}[\mathrm{X}]$ rixat & May 23,1977 & 9:00 a.m. \\
\hline
\end{tabular}

CURAENCE SUDJECT

TK-105-SX LIQUID LEVEL DECREASE EXCEEDING CRITERIA

1. OESCRIPTION OF OCEURAENCE ANO DESIGNATION OF APPARENT CAUSE

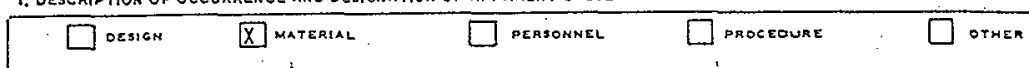

A baseline of 266.4 inches was established for 105-5X Tank on April 13, 1977. At 0900 on $5 / 23 / 77$ the liquid level measurement was 265.8 inches, exceeding the allowed variance from the baseline of .5 inch. (See attached table of liquid level. measurements.)

The apparent calise for the change in liquid level measurements is the heavily fissured crust of material on the surface of the liquid: The FIC instrument is not in contact with clear liquid. Liquid level measurements may then be expected to vary by more than one inch, depending upon the local topography of the crust.

CC: JC Cummings, EROA-RL

OJ Elgert, ERDA-RL (3)

PG Rhoades, ERDA-RL

JH Straub, ERDA-RL

\author{
bec: \\ DC Bartholomew \\ JM Cashrian \\ JL Deichnan \\ GT Dukelow \\ RA Freeman \\ JM Hajnes \\ DG Harlow \\ EJ Kosiancic \\ CH Nialody \\ $S$ Marchetti \\ GC Owens
}

RO Prosser

MF Rice

BJ Saueressig

HiP Shaw

SP Stalos

GH Upington/TL Bennington

iH Warren

AT White

JC Womack

RA Zinsti

Legal

Centra) File (Q-Safety 5.2

DJ Cockeram - Rockwell

2. OPERATING CONDITIONS OF THE FACILITY AT TIME OF OCCURRENCE (IF AFPLIGALLE)

Tank $705-5 X$ is considered sound and is currently used as a sturry receiver. No transfers of material into or out of the tank have occurred since April 12, 1977. This tank is not quarantined. 
Dry well data taken on $5 / 24 / 77$ showed no significant changes in the vertical radiation profiles.

Routine maintenance was performed on the FIC device on $5 / 17 / 77$. The reading after maintenance was .4 inch less than the reading before maintenance.

Photographs were taken on $5 / 24 / 77$. They reveal a heavily fissured surface layer of sait cake, typical of $241-5 X$ slurry tanks. The FIC plummet can be seen in an indentation in the surface.

RECOMHENDATIONS

A. TEMPORARY CORRECTIYE AETION

None.

B. PERMANENT CORRECTIVE AETION

None.

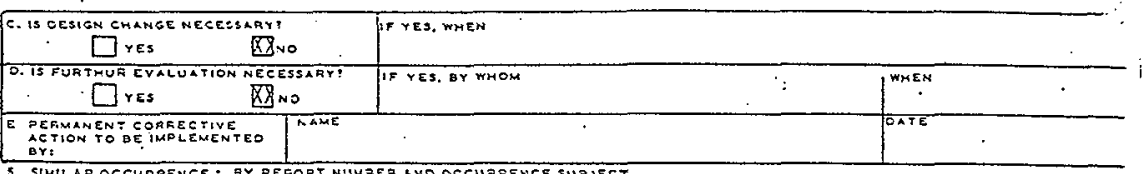

5. SIMILAR OCCURRENCE: EY REFORT NUMBEA AND OCCUARENCE SUBJECT

None.

\begin{tabular}{|c|c|}
\hline $\begin{array}{l}\text { ORIGINATEOBY } \\
\text { J. L. Deichman }\end{array}$ & Manager, Tank Farm Surveillance $\quad \cdot 6 / 6 / / 5 / 77$ \\
\hline 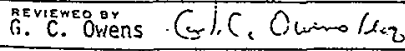 & Mánáger, Environmental and Occupational Sâtêty \\
\hline J. Mi Cashman TMCanhmem / he & Manager, Tank Farm Operations \\
\hline E. J. Kosiancic $\because:$ & Manager, Engineering \\
\hline A. T. White & Manager, Quality Assurance and Safety \\
\hline C. H. Malody & Máriager, Production and Waste Managementi/2//72 \\
\hline D. C. Bartholomew je.s br & Manager, Operations \\
\hline
\end{tabular}


HNF-SD-WM-ER-693, Rev. 0

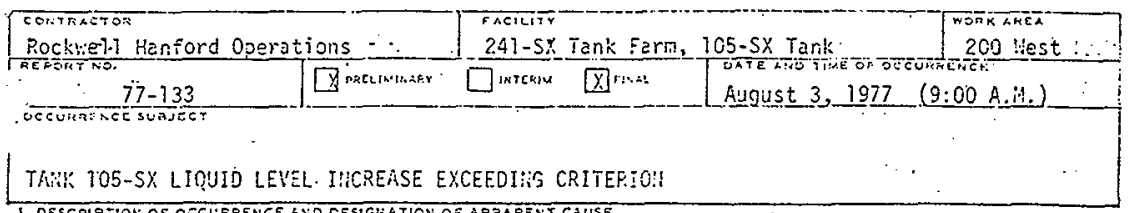

1. OESCPISTIOK OF OECLERENCEANO CESIGIATION OF́ APPARENTCAUSE

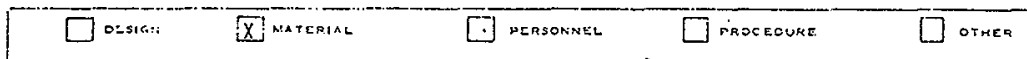

A baseline of 348.50 inches was establisingd for the 105-5x liquid level on 7-5-77. After four laeks of stearly grow'in, the linuid le:sl measurement reached 357.70 on 8-3-77, exceeding the allot:able 3 inch increase above baseline.

The apparent cause is volwa expansion, or srowti, of the tank materiat.

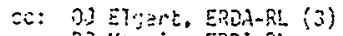

Sis :satris, BRA-72

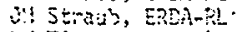

$\because$ Tigman, E?OA-?

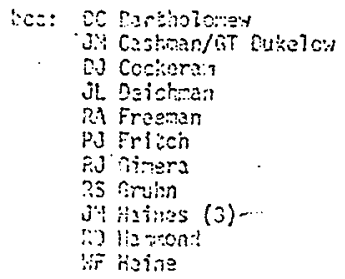

D) Cccisers:

it Dajerean

RA Freeman

PJ Frlich

S.j nimer2

25 Cruin

ol in ines (3)

(a) liz ron?

wir hisise

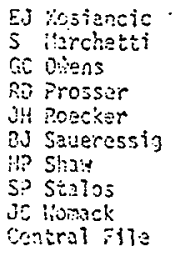

Ej Osiencic
S irchetti

cio oxens

Ro prosser

du Sazeressit

ith Shat:

Coneral Fita

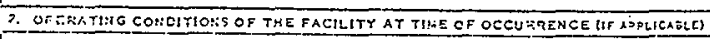

Tank 105-5x is a slumy-receivor for the 242-5 Evaporator Crystallizer. The tank is coistderes sound.

The tank is not quaratined.

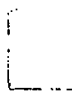


3. IYAEOATE EVALUATION, COREECTIVE ACTION TAKEN ANO RESULTS

Frequent flushes have produced only ainor, temporary decreases in the liquid level . : measurenent.

The growth phenomenon is frequently observed in all active $S X$ tanks. Superimposed on this general growth, ho:tever, are sudden drops attributed to cracks and crevices in. the thick, dry crust.

4. RECOMNENOATIONS

A. TEMPORARY CORRECTIVE ACYION

lione.

B. PERMAnENT CORRECTIVE action

None.

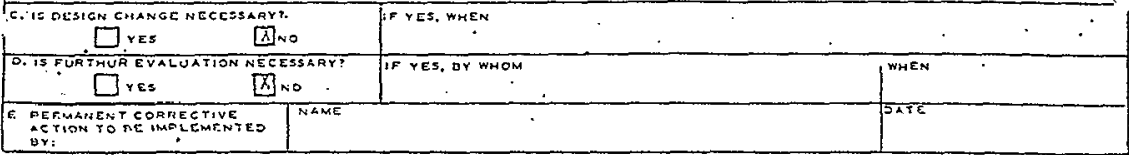

5. SIMULAR OCCURRENCE: GY REPORT HUMSER AND'OCCURRENCE SUAJECT.

Occurrence Report 77-74

"TAHK 105-SX LIQUID LEVEL DECREASE EXCEEOHIG CRITERIA"

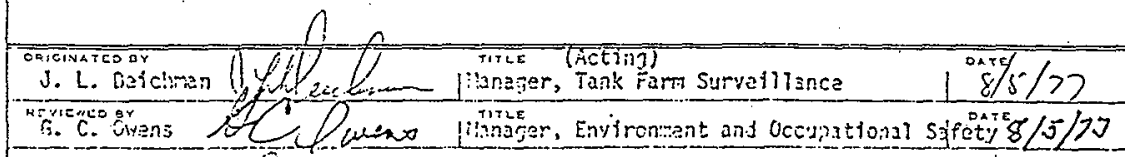

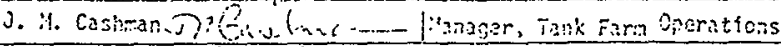

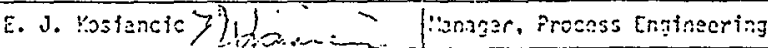

i. $\vec{F}$. lietre

Diractor, tiediti, Safety and Enylronisent

o. C. Eartiolgén pirector. Protuction operations

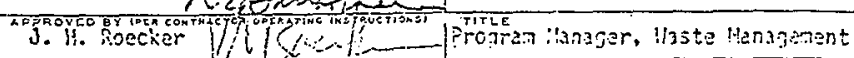

1.15177

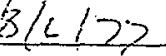

j

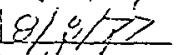

$8 / 8 / 7 ?$ 
HNF-SD-WM-ER-693, Rev. 0

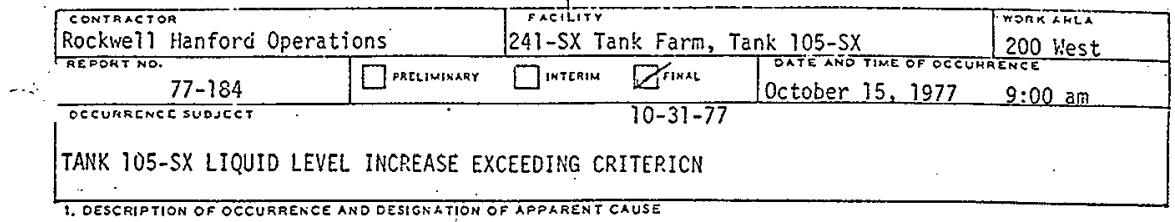

1. OESGRIPTION OF OCCURRENCE AND DESIGNATION OF APPARENT CAUSE

$\square$ Dogien $\square$ material $\square$ personnel $\square$ procesuke $\square$ other

A liquid level baseline of 354.60 inches was established for Tank 105-SX on September 28 , 1977. On October 15, 1977 the FIC licquid level measurement was 358.80 inches, exceeding the allowed 3.00 inch increase above the baseline.

The current liquid level measurement is 359.55 inches (10-28-77).

The increase is attributed to volume expansion of the slurry residing in the tank.

CC: JC Cummings, DOE

OJ Elgert, DOE (3)

$P G$ Harris, DOE

MW Tiernan, DOE

JH Straub, DOE bec: DC Bartholomew

JM Cashman/GT Dukelow

DJ Cockeran

JL Deichman

PJ Fritch

.JH Garbrick

RJ Gimera

RS' Gruhn

r.ju Haines (3)

RD. Hamond

hF lieine
EJ Kosiancic

GC Owens.

RD Prosser

JH Roecker

BJ Saueressig

HP Shav

SP Stajos

JA Swenson

JC Womack

Central File

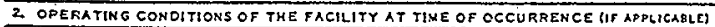

Tank 105-SX is considered sound. The tank receives siurry from the 242-S Evaporator Crystallizer.

This tank is not quarantined.

torek? 
3. IMAEDIATE EVALUATION, CORRECTIVE ACTIÓN TAKEN ANO OESULTS

Tank $105-5 X$ has a history of liquid level increases, attributed to volume expansion of the slurry.

$\therefore$ Manual tape readings are also taken in Tank 105-5X. From September 28 to October 15 the manual tape readings increased from 353.00 inches to 356.25 inches.

The possibility of inadvertent water addition to the tank has been investigated and discounted.

Photographs taken on 5-24-77 show a fissured crust over the entire surface. Photographs taken 10-19-77 show little change from those taken in liay.

4. RECOMMEN OATIONS

A.'TEMPORARY CORRECTIVE ACTION

None indicated at this time.

None indicated at this time.

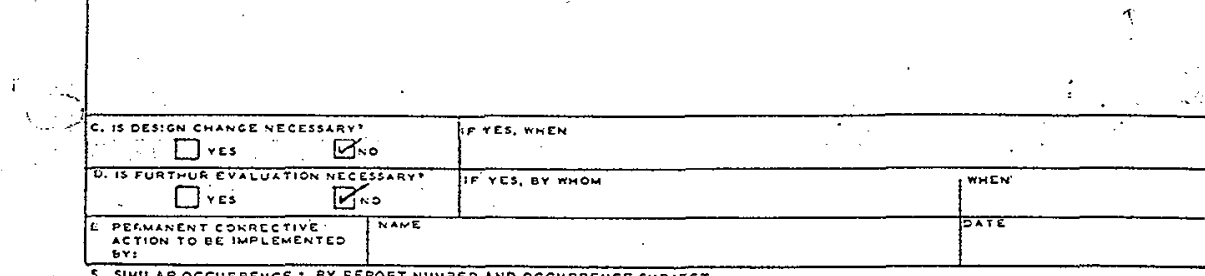

5. SIMTLAR OCCURRENCE: GY REPOAT NUMESR AND OCCURRENCE SUBJECT

77-133 - "Tank 105-SX Liquid Level Increase Exceeding Criterion"

76-172 - "Liquid Level Increase in Tank 105-SX Meeting Action Criteria"

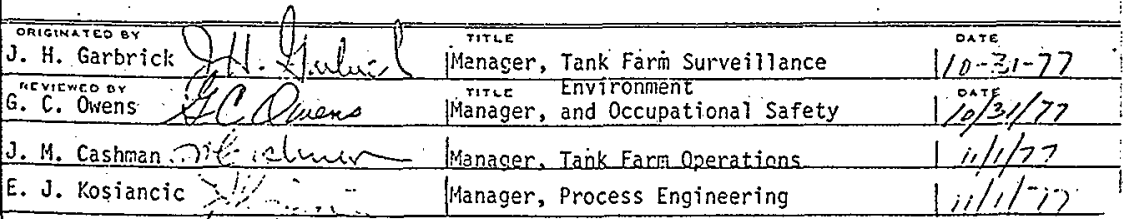

W. F. Heine 40 Quene for Director, Health, Safety and Environment

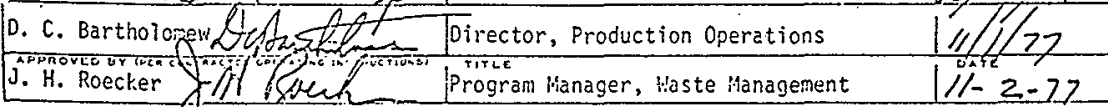

$4.3000 .918015 .7 e$ 


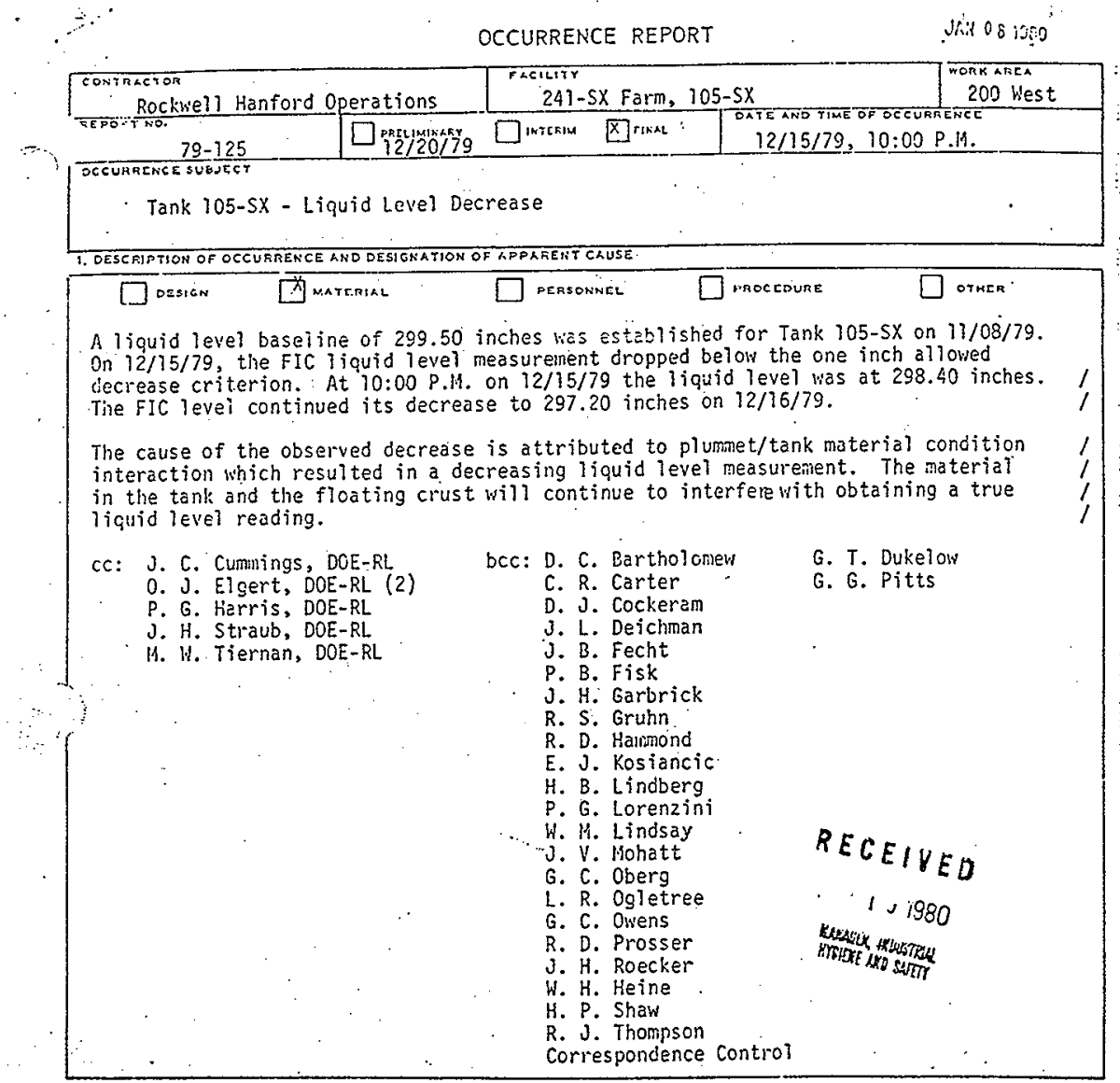

2. OPERATIMG CONDITIOLS OF THE FACILITY AT TIME OF OCCURRENCE \{IF APPLICABIC\}

Tank 105-5X was activated during October of 1979. It currently contains 810,000 gallons of waste. - The tank has 69,000 gallons of supernatant, 668,000 gallons of saltcake, and 73,000 gallons of sludge. The tank has lateral ory wells located 10 feet below the tank base.

The tank is currentiy pending reuse up to the 327 inch leve]. $\therefore$

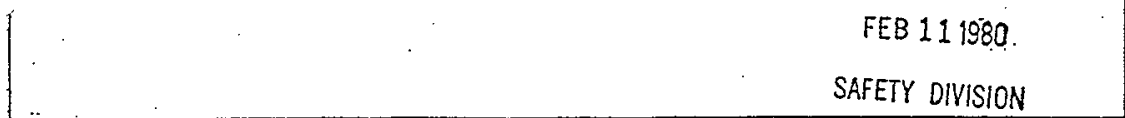

RECEIVED

FEB 111980.

SAFETY DIVISION 
Tank 105-5X was reacti:...ed during October 1979. Reuse of the tark was estiblished and thi tark was filled lip to the 300 inch ievel with waste from 107-5 on 10/18/79.

The tank material in 105-SX has historically exhibited a growth buildup pattern

(See Section 5). In-tank photographs were taken on $10 / 26 / 79$ in order to facilitate the establishment of a reuse liquid level baseline. The entire surface area consisted of a floating saltcake. The tank material appeared to have the consistency of spongelike foan.

Prior to the decrease of $12 / 15 / 79$, the tank's liquid level was exhibiting a slow growth increase. The leve was at 307.40 inches on $12 / 14 / 79$, the day prior to the decrease.

(continued on Attachient)

4. AECONMENDATIONS

A. TEMPORARY CORRECTIVE LCT:ON

8. PERMANENT COREECTIVE KETION

Flushed both the FIC and mianual liquid level tapes and plumets.

(Action: C. R. Carter, 01/09/80).

Established a new liquid level baseline for Tank 105-5X after the flush

(Action: C. R. Carter, 01/11/80).

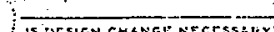

\begin{tabular}{|c|c|c|c|}
\hline 15 OCSIGN EHANGE NECESSARY? & IF res, NimEN & & \\
\hline 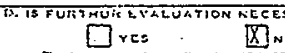 & IF, VES, BY WMDM & WHEN & \\
\hline 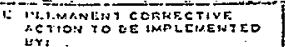 & C. R. Carter, Tank Farin Surveillance & $1 / 11 / 80$ & 1 \\
\hline
\end{tabular}

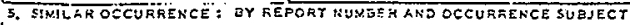

77-184, 10/15/77, Tank 105-5x Liquid Level Increase Exceeding Criterion.

77-133, 8/03/77, Tank 105-SX Liquid Level Increase Exceeding Criterion.

77-74, 5/23/77, Tank 105-SX Liquid Level Decrease Exceeding Criteria.

76-172; 12/15/76, Liquid Level Increase in Tank 105-5X Heeting Action Criteria.

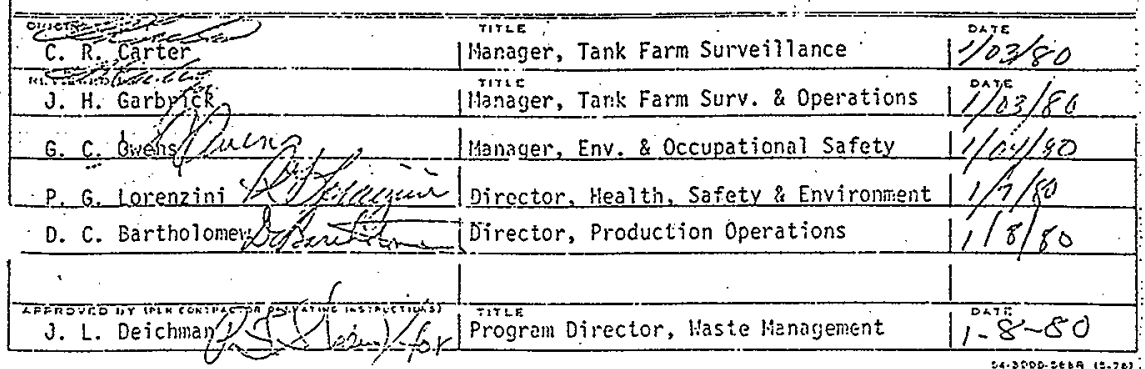


HNF-SD-WM-ER-693, Rev. 0

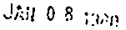

Attachment to 0. R. \$79-125 (Final)

\section{FACTS:}

In-tank photographs of 105-SX were taken on 12/17/79. A comparison between those photographs of the FIC tape/plumiet and the photographs taken on $70 / 26 / 79$ revealed the following:

- The FIC plummet photographed on $12 / 17 / 79$ was enbedded in the tank material. The plunmet was on the surface of the naterial in the $10 / 26 / 79$ photographs.

- The pluminet was relatively clean in the $10 / 26 / 79$ photographs while the exposed portion of the FIC plummet on 12/17/79 showed a material coating.

- The $12 / 17 / 79$ photographs show that the plumet bottom had a 2-i/2 inch long coating of green material. Above this coating there was a narrow light green band along the side of the plunimet.

- The 10/26/79 photographs show the surface as a relatively solid mass underneath the FIC plummet. The photographs of $12 / 77 / 79$ show the surface to be definitely floating and breaking up into a number of "islands". From the photographs, the floating crust material appears to be circulating counter clockwise relative to the FIC pluninet and tape.

- The $12 / 17 / 79$ pictures show the plumet to be tipped slightly, and making contact with crusty material.

- The laterals and vertical dry kells showed no change in radiation activity. The vertical dry wells were monitored on $12 / 17 / 79$ and $12 / 20 / 79$. The iaterals were monitored on $12 / 17 / 79,12 / 19 / 79$, and $12 / 21 / 79$.

The manual tape readings continue to vary between 24 feet, $10-3 / 4$ (298.75) inches and 24 feet, $11-1 / 4(299.25)$ inches, and the FIC readings continue to vary between 297.00 and 297.30 inches.

\section{CONCLUSIOHS:}

The cause of the observed decrease is attributed to a FIC plumnet/tank material condition interaction which resulted in a decreasing liquid level measurement. The FIC plummet began to dig a small indentation in the crust surface. The plummet, which contacts the surface once every hour, began to register a decreasing liquid level neasurement. This continuous hourly contact, aided by the crust movenent on the liquid surface most likely resulted in the crust breakup. The narrow light green band of material observed on the plumilet (approximately 4 inches from the botton) could have been the result of it contacting a piece of floating crust material. The naterial coating the plummet and the irreoular floating surface will continue to interfere with obtaining a true liquid level measurement in Tank 105-BX. The irregular monual tape recodings appear to be a resuit of an irregularly crusted surface. 


\begin{tabular}{|c|c|c|c|c|c|}
\hline \multicolumn{6}{|c|}{ DISTRIBUTION SHEET } \\
\hline \multirow{2}{*}{$\begin{array}{l}\text { To } \\
\text { Distribution }\end{array}$} & \multirow{2}{*}{\multicolumn{3}{|c|}{$\begin{array}{l}\text { From } \\
\text { C. H. Brevick }\end{array}$}} & \\
\hline & & & & \multicolumn{2}{|c|}{$\frac{\text { Page } 1 \text { of } 1}{\text { Date June } 24,1997}$} \\
\hline \multicolumn{4}{|c|}{ Project Title/Work Order } & \multicolumn{2}{|c|}{ EDT No. 156939} \\
\hline \multicolumn{4}{|c|}{$\begin{array}{l}\text { Flammable Gas Tank Waste Level Reconciliation, Tank 241-SX-105 / } \\
\text { E40697 }\end{array}$} & \multicolumn{2}{|l|}{ ECN No. } \\
\hline Name & MSIN & $\begin{array}{c}\text { Text } \\
\text { With All } \\
\text { Attach. }\end{array}$ & Text Only & $\begin{array}{l}\text { Attach./ } \\
\text { Appendix } \\
\text { Only }\end{array}$ & $\begin{array}{l}\text { EDT/ECN } \\
\text { Only }\end{array}$ \\
\hline
\end{tabular}

Duke Engineering and Services Hanford
R. E. Bauer
R. J. Cash
T. C. Geer
G. D. Johnson

$57-14$

S7-14

R1-43

S7-14

$x$
$x$
$x$
$x$

Fluor Daniel Northwest

C. H. Brevick

L. A. Gaddis

B. D. Andres

R. P. Schwehr

* Engr. Publications

$\begin{array}{ll}E 6-08 & X \\ H 5-57 & X \\ E 6-08 & X \\ E 6-08 & X \\ E 6-63 & \end{array}$

Lockheed Martin Hanford Corporation
S. A. Barker
W. B. Barton
J. G. Kristofzski
B. C. Simpson
A. E. Young

\section{Lockheed Martin Services}

Document Control

Meier Associates

J. D. Bingham

D. C. Hedengren
R2-11

R2 -12

R2 -12

R2-12

R1-10

G3-11 $\quad X$

$\begin{array}{ll}R 2-11 & X \\ R 2-11 & x\end{array}$

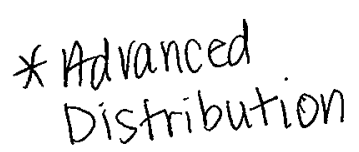

